University of San Diego

Digital USD

1999-04-01

\title{
Factors Related to Stage at Diagnosis in African-American and Caucasian Women Diagnosed with Breast Cancer in the Military Health System
}

Sandra C. Garmon Bibb DNSc, MSN, RN

University of San Diego

Follow this and additional works at: https://digital.sandiego.edu/dissertations

Part of the Nursing Commons

\section{Digital USD Citation}

Garmon Bibb, Sandra C. DNSc, MSN, RN, "Factors Related to Stage at Diagnosis in African-American and Caucasian Women Diagnosed with Breast Cancer in the Military Health System" (1999). Dissertations. 284.

https://digital.sandiego.edu/dissertations/284

This Dissertation: Open Access is brought to you for free and open access by the Theses and Dissertations at Digital USD. It has been accepted for inclusion in Dissertations by an authorized administrator of Digital USD. For more information, please contact digital@sandiego.edu. 
UNIVERSITY OF SAN DIEGO

Hahn School of Nursing and Health Science

DOCTOR OF NURSING SCIENCE

FACTORS RELATED TO STAGE AT DIAGNOSIS IN AFRICAN AMERICAN AND

CAUCASIAN WOMEN DIAGNOSED WITH BREAST CANCER IN THE MILITARY

HEALTH SYSTEM

by

Sandra C. Garmon Bibb, MSN, RN

A dissertation presented to the

FACULTY OF THE HAHN SCHOOL OF NURSING AND HEALTH SCIENCE

UNIVERSITY OF SAN DIEGO

In partial fulfillment of the

requirements for the degree

DOCTOR OF NURSING SCIENCE

April 1999 


\begin{abstract}
The focus of this descriptive-comparative study was to investigate the relationships between potential access (race, age, socioeconomic status (SES), and the means of discovery of breast cancer), realized access (the length of time between discovery of breast abnormality and diagnosis of breast cancer, and the length of time between diagnosis and initiation of breast cancer treatment), and stage at diagnosis of breast cancer, in African American and Caucasian women, receiving care within an equal economic access health care system. The research questions were answered through secondary analysis, using data obtained from the tumor registry records of 62 African American and 573 Caucasian women diagnosed with breast cancer within the Department of Defense Military Health System, from January l, 1988 through December 31, 1997. Descriptive statistics were used to describe the sample and the study variables; and independent samples chi-square tests, t-tests, Mann Whitney U tests, analysis of variance, multiple regression, and logistic regression were used to analyze the data. Results indicate that, when compared to Caucasian women, African American women: (a) were diagnosed at a significantly later stage of breast cancer, (b) were significantly younger at the time of diagnosis, (c) were from a significantly lower SES, (d) had a significantly higher percentage of incidental breast self exam (BSE) discovered breast abnormalities, and (e) had a significantly lower percentage of mammogram discovered breast abnormalities. Women with breast abnormalities discovered by incidental BSE were almost six times more likely to be diagnosed in late stage breast cancer than women with mammogram discovered breast abnormalities. There were no significant differences noted between African American and Caucasian women in length of time between discovery and diagnosis, and length of time between diagnosis and treatment.
\end{abstract}




\section{DEDICATION}

I would like to dedicate this dissertation to:

The memory of my father, Elijah Garmon Sr., whose strong work ethic and tenacious approach to life taught me to persevere, and encouraged me to purpose to succeed.

The memory of my brother-in-law, Dr. Levi B. Baldwin Jr., whose confidence in me and in who God created me to be gave me the confidence to navigate uncharted waters.

The memory of Eloise Lewis, whose battle with breast cancer intensified my query of factors related to access and stage at diagnosis of breast cancer in African American women. 


\section{ACKNOWLEDGMENTS}

I would like to express my sincere appreciation and thanks to those individuals who supported me during this endeavor.

To my dissertation committee:

Dr. Jane Georges, my chair, for her example, expertise, experience and encouragement.

Dr. Jan Harrison, for her expertise, enthusiasm, experience, and invaluable suggestions.

Dr. Peter Johnstone, for his mentorship, friendship, expertise, and enthusiasm.

To Dr. R. H. Riffenburgh, for his statistical expertise and methodological guidance.

To the tumor registrar assistants at Naval Medical Center San Diego, for their invaluable assistance, encouragement, and creation of an enthusiastic environment for data collection.

To my family:

My daughter and son, Monica and Rodney Moss, for their love, patience, and emotional support.

My grandchildren, Meghan and Micah Moss, for their creation of healthy, happy, distractions on cue. 
My sisters, Etta Baldwin and Jacquelyn Ruffin, for their love, example, support, confidence, and encouragement.

My mother, Mary Garmon and my brother, Elijah Garmon Jr., for their love, support, and inspiration.

My nieces and nephews for their confidence and encouragement.

My Pastors, Russell and Barbara Plilar, for their emotional support, love, confidence, encouragement, and enthusiasm.

Seedtime and Harvest Church for their love, confidence, and encouragement.

To Cindy Lopez and Robin McKenzie, for their friendship and editorial assistance.

To my professors, for their example, encouragement, and enthusiasm.

To the United States Navy Nurse Corps for providing me with the opportunity to pursue this long-held goal.

Most of all, to God, Whose Strength enabled me to achieve this goal in excellence, with strength, and with confidence. 
Abstract

Dedication

ii

Acknowledgments

List of Figures

List of Tables

List of Appendices

\section{Chapter}

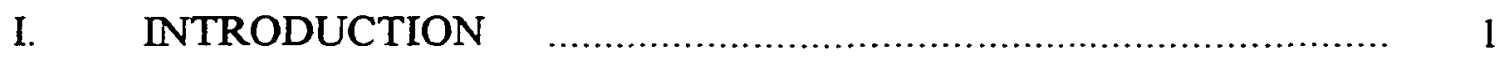

Statement of Problem ..................................................... 4

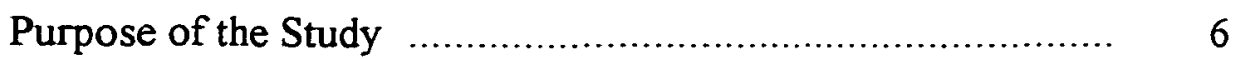

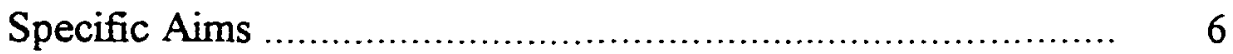

Conceptual Framework ................................................... 8

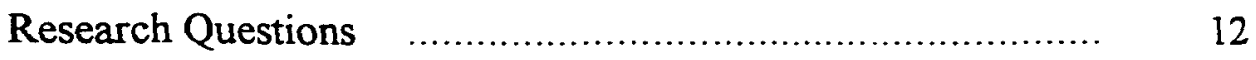

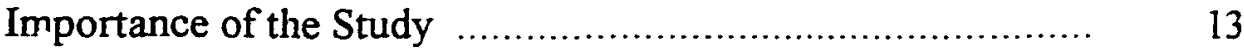

Definition of Terms ….................................................. 15

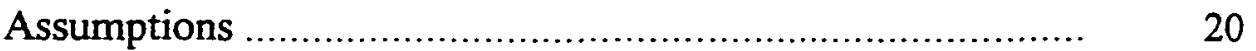

II. REVIEW OF THE LITERATURE _......................................... 21

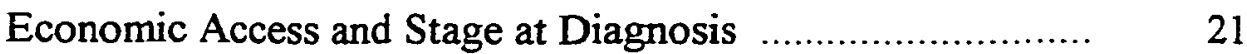

Potential Access and Stage at Diagnosis ……....................... 29

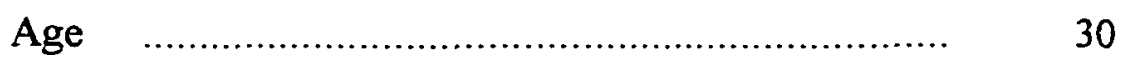




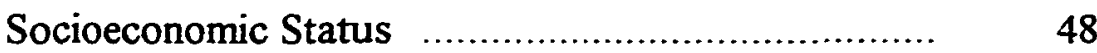

Means of Discovery of Breast Cancer....................... 53

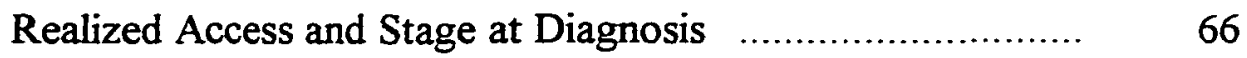

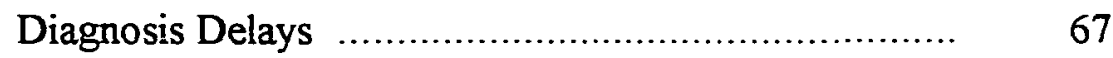

Treaiment Delays …........................................ 71

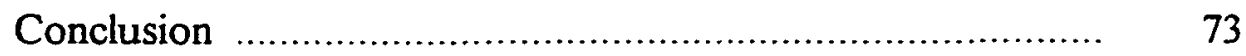

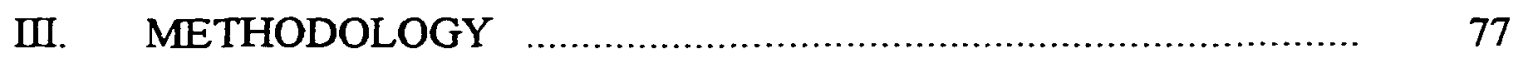

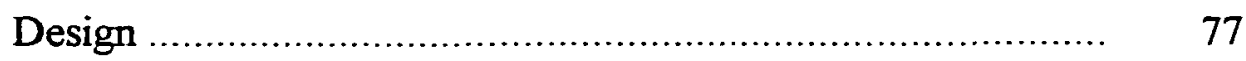

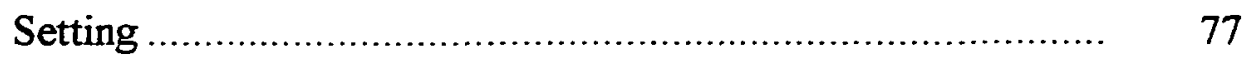

Population and Sample …......................................... 80

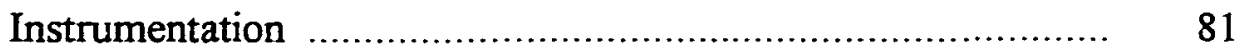

Data Collection and Recording …….................................... 81

Data Analysis . ............................................................... 83

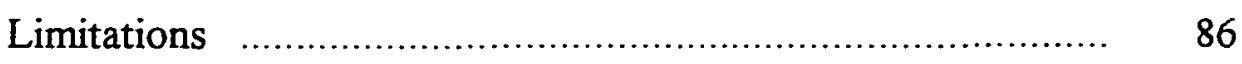

Human Subjects $\quad$............................................................. 87

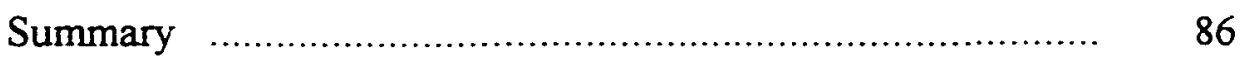

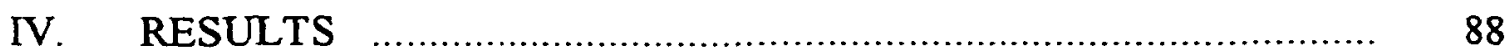

Description of the Sample

Descriptive Analysis of the Study Variables …….................. 96

Data Analysis Related to the Research Questions.................... 101

Research Question 1............................................ 101 


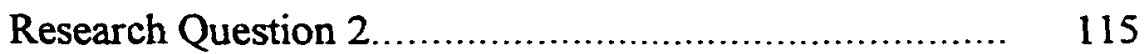

Research Question 3 ......................................... 120

Research Question 4........................................... 125

Research Question 5 ........................................ 130

Presentation of Results from Supplemental Data Analysis.......... $\quad 130$

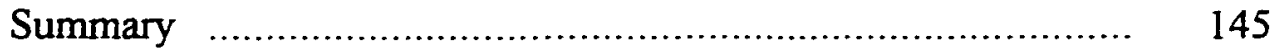

V. SUMMARY, DISCUSSION, IMPLICATIONS, AND CONCLUSION ....................................................... 146

Summary of the Study .....................................................

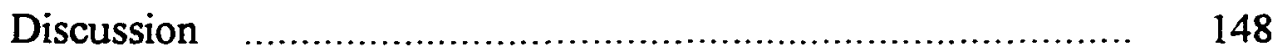

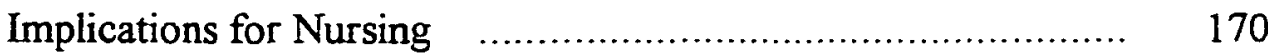

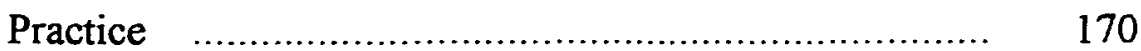

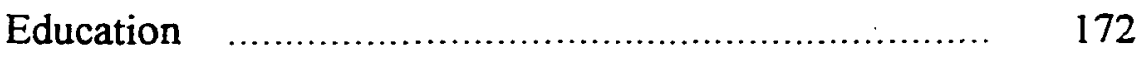

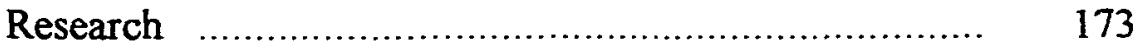

Conclusion

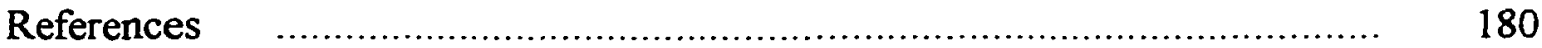

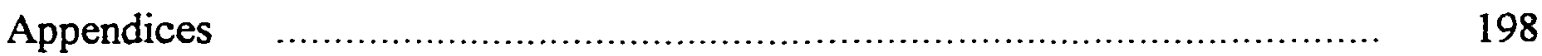




\section{List of Figures}

Figure

Page

1. Framework for the Study of Access to Breast Health Care

for Women in the Military Health System

11

2. Revised Framework for the Study of Access to Breast Health Care for Women in the Military Health System 
List of Tables

Table

Page

1. American Joint Committee on Cancer Primary

Tumor Classification

2. American Joint Committee on Cancer Lymph

Nodes Classification

3. American Joint Committee on Cancer Distant

Metastasis Classification

4. American Joint Committee on Cancer Clinical

Staging of Breast Cancer

5. Summary of Demographic and Study Variable

Characteristics

6. Distribution of Demographic and Study

Variable Characteristics By Race

7. Relationship Between Stage at Diagnosis

and Age at Diagnosis

8. One Way Analysis of Variance of Mean Age

at Diagnosis and Stage at Diagnosis By Race

9. Relationship Between Stage at Diagnosis and

Age at Diagnosis By Race

10. Relationship Between Stage at Diagnosis and Race

11. Relationship Between Stage at Diagnosis and

Rank of Sponsor

12. Relationship Between Stage at Diagnosis and

Means of Discovery

13. Distribution of Rank of Sponsor By Race

14. Distribution of Means of Discovery By Race 
15. Relationship Between Stage at Diagnosis and Length of Time Between Discovery and Diagnosis

16. Relationship Between Stage at Diagnosis and Length of Time Between Diagnosis and Treatment

17. Relationship Between Age at Diagnosis and Variables of Realized Access

18. General Regression of Age at Diagnosis, Race, SES, and Means of Discovery on Length of Time Between Discovery and Diagnosis

19. Intercorrelation Matrix of Variables of Potential Access (Length of Time Between Discovery and Diagnosis)

20. General Regression of Age at Diagnosis, Race, SES, and Means of Discovery on Length of Time Between Diagnosis and Treatment

21. Intercorrelation Matrix of Variables of Potential Access (Length of Time Between Diagnosis and Treatment)

22. Logistic Regression Analysis of Late Stage at Diagnosis as a Function of Variables of Potential Access

23. Logistic Regression Analysis of Late Stage at Diagnosis as a Function of Variables of Realized Access

24. Relationship Between Variables of Potential Access and Means of Discovery

25. Distribution of Variables of Potential Access By Age

26. T Test Summary Table of Selected Study Variables By Means of Discovery

27. Logistic Regression Analysis of Provider Discovered Breast Abnormalities as a Function of Selected Demographic Variables

28. Distribution of Means of Discovery, By Year 


\section{List of Appendices}

Appendix

Page

A. Breast Cancer Medical Record Data Collection Sheet

B. Human Subjects Letter, Naval Medical

Center San Diego

C. Human Subjects Letter University of

San Diego

202 


\section{CHAPTER 1}

\section{INTRODUCTION}

Breast cancer is a major medical problem affecting the quality of life of all American women. Second to lung cancer as the leading cause of cancer death in women in the United States, breast cancer occurs in American women more often than any other type of cancer. From 1993 through 1995, calculated over a life time, one in every 18 women developed invasive cancer of the lungs and bronchus, while one in every 8 women developed cancer of the breast (Landis et al., 1999). In 1999, approximately 175,000 American women will be newly diagnosed with breast cancer, and an estimated 43,300 will die of this invasive disease (Landis, Murray, Bolden, \& Wingo, 1999).

Healthy People 2000, published in 1990, set three broad goals for all Americans: increasing the span of healthy life, reducing health disparities, and achieving access to preventive services (Department of Health and Human Services [DHHS], 1990). Healthy People 2000 set a specific goal of reducing breast cancer death rates from an age adjusted baseline of 22.9 per 100,000 women in 1987 , to no more than 20.6 per 100,000 women by the year 2000. In addition, Healthy People 2000 acknowledged a disparity in breast cancer death rates between White women and women of color; and targeted women of color for increased preventive services relating to breast cancer (DHHS, 1990).

The United States Bureau of Census reported (as cited in Parker, Davis, Wingo, Ries, \& Heath, 1998), that in 1990, White Americans constituted about 84 percent of the 
United States population. African Americans made up approximately 12 percent of the population; and Hispanics constituted about 9 percent. Asian and Pacific Islanders comprised about 3 percent of the United States population in 1990. Composition of this group included Chinese, Filipino, Hawaiian, Japanese, Korean, and Vietnamese Americans. Native Americans made up about 0.8 percent of the United States population in 1990, and included American Indians and Alaska Natives. From 1990 to 1995, the age adjusted breast cancer incidence rates for women in the United States, by race and ethnicity per 100,000 were: (a) 113.2 for White women, (b) 99.0 for African American women, (c) 71.4 for Asian/Pacific Islander women, (d) 69.3 for Hispanic women, and (e) 31.9 for American Indian women. However, the age adjusted breast cancer mortality rates for women by race and ethnicity per 100,000 from 1990 to 1995 were: (a) 31.5 for African American women, (b) 26.0 for White women, (c) 15.3 for Hispanic women, (d) 11.7 for American Indian women, and (e) 11.6 for Asian/Pacific Islander women (Landis et al., 1999). Even though White women have the highest incidence of breast cancer in the United States and the mortality rate cited above for White women is well above the Healthy People 2000 goal of 20.6 per 100,000 women, the breast cancer mortality rate in African American women is higher than the mortality rate in White women, and in all other groups of women of color.

Because of this disparity in mortality rate between African American and White women, and because African American women comprise the largest ethnic minority group in the United States, current research examining breast cancer disparity between White women and women of color has focused primarily on African American women. From 
1989 to 1993, age adjusted breast cancer mortality rates in the United States for White women decreased by approximately six percent, while breast cancer mortality rates rose about one percent for African American women (National Cancer Institute [NCI], 1996). Hence, breast cancer impacts achievement of the Healthy People 2000 goal of increasing the span of healthy life for all American women; however, the disproportionate influence of breast cancer mortality on the lives of African American women threatens to also impede achievement of the Healthy People 2000 goal of reduced disparities in health care. Delayed diagnosis is consistently cited as the cause of the disparity in breast cancer mortality between White women and African American women (Bedell, Wood, Lezotte, Sedlacek, \& Orleans, 1995; Hunter et al., 1993; Long, 1993; Mandelblatt, Andrews, Kao, Wallace \& Kerner, 1995; Simon \& Severson, 1996; Thompson, Montano, Mahloch, Mullen, \& Taylor, 1997). From 1989 to 1994, 50 percent of the African American women diagnosed with breast cancer presented with localized tumors, as compared to 62 percent of the White women diagnosed; and the five year breast cancer survival rate for White women was 87 percent as compared to 71 percent for African American women (Landis et al., 1999).

Access barriers have been identified as the primary cause of diagnosis delay (Ansell, Lacey, Whitman, Chen, \& Phillips, 1994; Ayanian, Kohler, Abe, \& Epstein, 1993; Lauver, Coyle \& Panchmatia, 1995). Studies exploring access as a barrier to cancer care and screening have defined access primarily in terms of availability of care, or in relation to economic factors and health insurance coverage (Ayanian et al., 1993; Mandelblatt, Andrews, Kao, Wallace, \& Kerner, 1995; Mandelblatt, Andrews, Kerner, Zauber, \& 
Burnett, 1991; Michels, Taplin, Carter, \& Kugler; 1995; Zaloznik, 1995, 1997). As a result, providing economic access to breast health care has become the primary focus for: increasing African American women's participation in breast cancer screening programs, eliminating delayed diagnosis, and decreasing breast cancer mortality in African American women.

The Department of Defense (DoD) Military Health System (MHS) provides "equal" health care access to all active duty and retired members of the Armed Forces, and their family members (Assistant Secretary of Defense for Health Affairs [ASDHA], 1996a). Race and socioeconomic status are not a factor in determining who will receive care. Thus, if economic access to care is the primary factor creating delayed diagnosis of breast cancer, there should be no significant difference in stage at diagnosis of breast cancer between White women and African American women receiving care within the MHS.

\section{Statement of the Problem}

Research is needed to describe the relationship between access and stage at diagnosis for women being treated for breast cancer inside the MHS. The 1996 National Defense Authorization Act allocated 25 million dollars for breast cancer prevention and treatment within the DoD MHS (Assistant Secretary of Defense for Health Affairs [ASDHA], 1996b). The result of this legislation was twofold: development of the DoD Breast Cancer Prevention, Diagnosis, and Education Initiative; and movement of breast cancer prevention, diagnosis, and treatment to the forefront of DoD MHS health promotion and disease prevention strategies. Thus, the overall objective of the DoD's 
breast cancer initiative was "to ensure awareness of, and easy access to, superior medical and diagnostic technology and follow-up care for the early detection and optimum treatment of breast cancer in active duty women and women beneficiaries" (ASDHA, 1996b, p.2). Within the DoD MHS there are varying levels of rank and therefore varying levels of socioeconomic strata. Economic access to health care, however, is purported to be "equal" despite rank or socioeconomic status. Therefore, the disparity in breast cancer stage at diagnosis between White women and African American women in the larger population, attributed to lack of economic access to breast health care, is expected to be nonexistent inside the DoD MHS. Furthermore, the existence of government funding for breast health care in the MHS could lead policy makers and providers of health care to conclude that "...easy access to, superior medical and diagnostic technology and follow-up care for the early detection and optimum treatment of breast cancer.." exists for all women receiving breast health care in the MHS.

The year 2000 is rapidly approaching and breast cancer continues to attack all American women. Successful achievement of the Healthy People 2000 goal of reduced disparity in breast cancer mortality is contingent upon a reduced mortality rate in African American women. If economic access to breast health care is the key to combating breast cancer mortality and eliminating stage at diagnosis disparity between White women and women of color, then all such legislation and policy issuance should focus on providing economic access to breast health care for all women. However, if significant disparity exists in breast cancer stage at diagnosis between White women and women of color in an 
"equal" access system, then economic access must be eliminated as the primary cause of the disparity, and other contributors to delayed diagnosis must be identified.

Purpose of the Study

The purpose of this study was to describe the relationship between access and stage at diagnosis of breast cancer in African American and Caucasian women being diagnosed with breast cancer within the DoD MHS. Relationships between stage at diagnosis of breast cancer and age, socioeconomic status, the means of discovery of breast cancer, the length of time between discovery of breast abnormality and diagnosis of breast cancer, and the length of time between diagnosis and initiation of breast cancer treatment, were explored to provide relevant knowledge concerning the interrelationships between these variables in women receiving care within the MHS.

African American women and White women were the focus of this research study. The term African American women is used interchangeably with the term Black women in the health care literature; therefore these terms were used interchangeably in this study. The term Caucasian women is used interchangeably with the term White women in the health care literature; therefore these terms were also used interchangeably in this study.

Specific Aims

The specific aims of this study were to:

1) Describe the age and socioeconomic status (defined as rank) of women identifying their race as Caucasian or African American and being diagnosed with breast cancer at Naval Medical Center San Diego from January 1, 1988 to December 31, 1997. 
2) Describe the means of discovery of breast cancer (breast self exam, clinical breast exam, mammogram) in women identifying their race as Caucasian or African American and being diagnosed with breast cancer at Naval Medical Center San Diego from January 1, 1988 to December 31, 1997.

3) Identify the length of time in days between discovery of breast lump, mass, or abnormality and diagnosis of breast cancer, and between diagnosis of breast cancer and initiation of treatment, in women identifying their race as Caucasian or African American and being diagnosed with breast cancer at Naval Medical Center San Diego from January 1, 1988 to December 31, 1997.

4) Identify the stage at diagnosis of breast cancer in women identifying their race as Caucasian or African American and being diagnosed with breast cancer at Naval Medical Center San Diego from January 1, 1988 to December 31, 1997.

5) Compare the relationship between age, socioeconomic status, race and the means of discovery of breast cancer, length of time before diagnosis, length of time between diagnosis and initiation of treatment, and stage at diagnosis in women identifying their race as Caucasian and being diagnosed with breast cancer at Naval Medical Center San Diego from January 1, 1988 to December 31, 1997 with the relationship between age, socioeconomic status, race, and the means of discovery of breast cancer, length of time before diagnosis, length of time between diagnosis and initiation of treatment, and stage at diagnosis in women identifying their race as African American and being diagnosed with breast cancer at Naval Medical Center San Diego from January 1, 1988 to December 31, 1997. 


\section{Conceptual Framework}

The conceptual framework for this study was based on the Aday/Andersen Framework for the Study of Access (Aday \& Andersen, 1974). The Aday/Andersen access model was initially applied directly in a national survey of access to medical care conducted in 1976 (Aday, Andersen, Fleming, 1980). This model has subsequently provided the framework for numerous community surveys of access to medical care (Aday, 1985; Aday, et al., 1993; Andersen, Giachello, \& Aday, 1986; Northam, 1996; Urrutia-Rojas \& Aday, 1991), and studies evaluating the impact of access barriers on health care (Aday, Andersen, Loevy, \& Kremer, 1984; Champion \& Menon, 1997; Guidry, Aday, Zhang, \& Winn, 1997; Guidry, et al., 1996; Miller \& Champion, 1997).

The Aday/ Andersen framework defines access as "...those dimensions which describe the potential and actual entry of a given population group to the health care delivery system" (Aday, Fleming, \& Andersen, 1984, p. 13). Health care policy is characterized as the starting point for access in this model since improved access is considered to be a primary goal of most health care policies (Aday \& Andersen, 1974). Indicators of potential access are identified as characteristics of the health care delivery system and the population at risk, while indicators of actual or realized access are identified as utilization and satisfaction. More specifically, the likelihood of an individual's entering the health care delivery system is purported to be influenced by the structure of the system, and the nature of the needs, wants, and resources brought to the care-seeking process by potential consumers of health care; and the realization of entrance into the health care delivery system is demonstrated by a population's reported rates of utilization, 
and through their subjective evaluation of the health care services they obtain (Aday, Fleming, \& Andersen, 1984).

In conceptualizing potential access, characteristics of the health care delivery system are operationalized as the availability and organization of health care resources (Aday, Andersen, \& Fleming, 1980). Health care resources include health care personnel, structures in which the health care is delivered, the education provided, and equipment and materials used in providing the care. Characteristics of the population at risk are operationalized as predisposing, enabling, and need-oriented. Predisposing characteristics include such variables as age, sex, race, and values concerning health and illness, and describe the "propensity" of individuals to use health care services. Enabling characteristics include such variables as income, insurance coverage, and geographic location in relation to the health care services, and describe the "means" individuals have at their disposal for the use of services. The need for health care may be either perceived by the individual or determined by the health care delivery system, and refers to the most immediate initiator of health care service use, the illness level (Aday, Andersen, \& Fleming, 1980).

Realized access is conceptualized by characteristics of the utilization of services and consumer satisfaction (Aday, Andersen, \& Fleming, 1980). Utilization of health care services can be objectively measured and is operationalized by evaluation of the type of services utilized, the site of the health care encounter, the purpose of the utilization of services, and the time interval involved with utilization of services. The time interval can be evaluated in terms of whether or not an individual entered the health care system within 
a given period of time, by the number of visits within a given period of time, or by the degree of coordination of services associated with a particular illness. Consumer satisfaction is operationalized as a subjective measure of the consumer's attitudes toward the health care delivery system and their satisfaction with their experiences with the health care delivery system (Aday, Andersen, \& Fleming, 1980).

This study did not investigate all of the variables described by Aday and Andersen (1974). Data were collected regarding the age, race, and socioeconomic status of the population at risk. Variables characterizing the event initiating utilization of specialized breast health care services were evaluated. Aspects of utilization of health care services were also evaluated. A schematic model of the variables selected for inclusion in this study is presented in Figure 1. The arrows depict the flow of influence theorized by the investigator to exist between potential and realized access, and stage at diagnosis of breast cancer. This conceptual model also provided the framework for the literature review presented in chapter two. 
Figure 1. Framework for the Study of Access to Breast Health Care for Women in the Military Health System

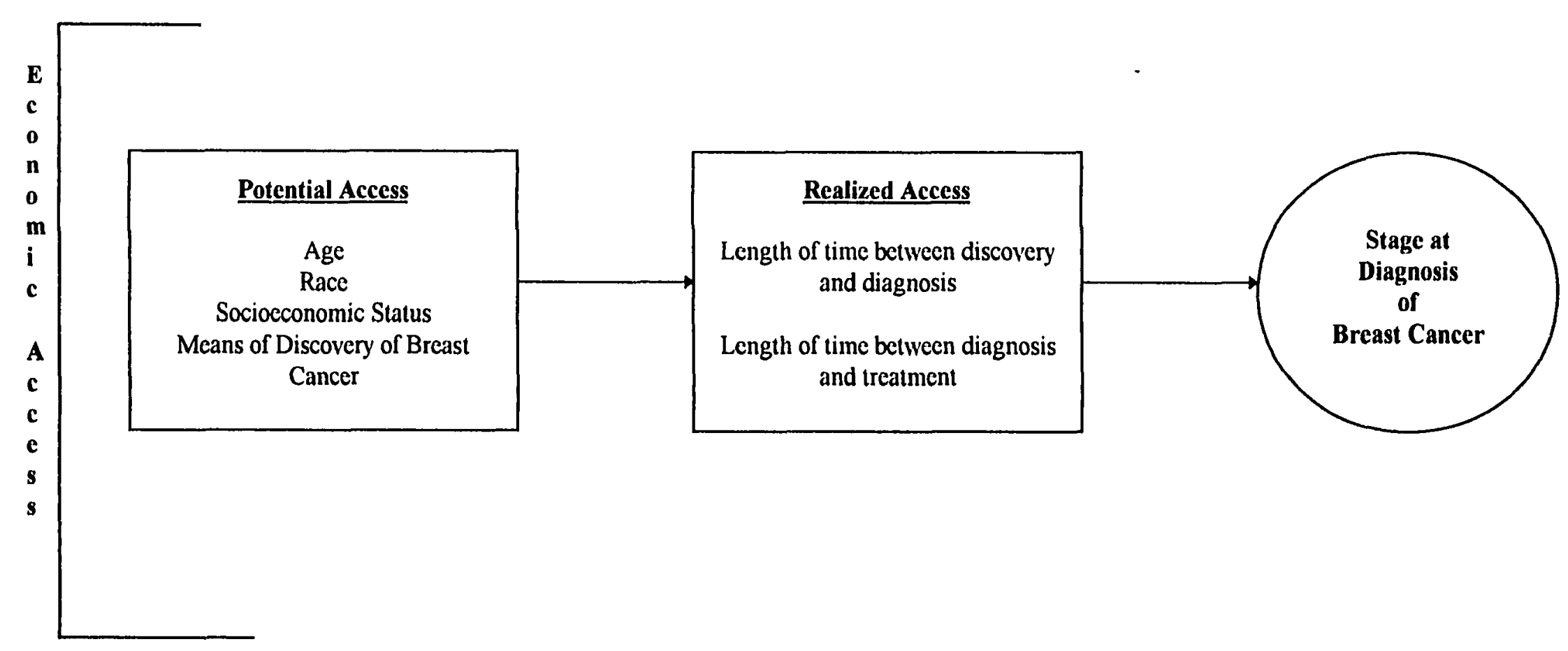

Adapted from Aday/Andersen Framework for the Study of Access (1974) 


\section{Research Questions}

1. What are the relationships between age, race, socioeconomic status, the means of discovery of breast cancer, and stage at diagnosis of breast cancer in women identifying their race as Caucasian or African American, and being diagnosed with breast cancer at Naval Medical Center San Diego from January 1, 1988 to December 31, 1997.

2. What are the relationships between the length of time between discovery and diagnosis of breast cancer, and the length of time between diagnosis and initiation of treatment of breast cancer, and stage at diagnosis of breast cancer in women identifying their race as Caucasian or African American, and being diagnosed with breast cancer at Naval Medical Center San Diego from January 1, 1988 to December 31, 1997.

3. To what extent do the variables age, race, socioeconomic status, and the means of discovery of breast cancer predict the length of time between discovery and diagnosis of breast cancer, and the length of time between diagnosis and initiation of treatment of breast cancer, in women identifying their race as Caucasian or African American, and being diagnosed with breast cancer at Naval Medical Center San Diego from January 1, 1988 to December 31, 1997.

4. To what extent do the variables age, race, socioeconomic status, and the means of discovery of breast cancer predict stage at diagnosis of breast cancer in women identifying their race as Caucasian or African American, and being diagnosed with breast cancer at Naval Medical Center San Diego from January 1, 1988 to December 31, 1997.

5. To what extent do the variables length of time between discovery and diagnosis of breast cancer, and length of time between diagnosis and initiation of treatment of breast 
cancer, predict stage at diagnosis of breast cancer in women identifying their race as Caucasian or African American, and being diagnosed with breast cancer at Naval Medical Center San Diego from January 1, 1988 to December 31, 1997.

\section{Importance of the Study}

Addressing the problem of access by focusing principally on availability of care, economic barriers, and health insurance coverage, excludes the impact of other aspects of access, and assumes that economic access to care translates into utilization of health care services (Bashshur, Homan, \& Smith, 1994; Facione \& Facione, 1997; Friedman, 1994; Guidry et al., 1996; Pearlman, Rakowski, Ehrich, \& Clark, 1996).

Steiner (1991), states:

Access to health care is more than the mere availability of physicians and county hospitals or clinics to serve the medically indigent within a certain geographic area; it is the availability of services that are financially accessible to the patient; it is the availability of effective cancer prevention and early detection educational messages that are culturally sensitive and meaningful to the patient population; and it is the availability of acceptable, appropriate, and comprehensive early detection and treatment services in an environment that is concerned about the needs of the person, treats the person with dignity, provides care in a timely fashion, and provides quality, culturally sensitive care (p. 1736).

The success of health care service availability in decreasing morbidity and mortality associated with cancer depends on actual utilization of services (Mandelblatt et al., 1993); 
and utilization of cancer screening services is guided by a perception of their necessity and appropriateness, as well as their availability.

The existing nursing research on African American women's access to breast health programs has been inadequate in its provision of knowledge of factors influencing African American women's participation in breast health care (Bibb, 1997). This inadequacy presents a practice problem for the discipline of nursing, since the primary purpose of nursing research is to improve nursing practice (Bunting \& Campbell, 1994; Hunt, 1996); and nursing's involvement in African American women's participation in breast health programs is crucial if breast cancer mortality is to be reduced. "Research based knowledge is incorporated into one's critical thinking about identified practice problems and changes are made in the individual's personal professional practice on the basis of this knowledge" (Crane, 1995). Therefore, continuing to focus current research on testing availability of care and health care insurance, as an exclusive determinant of African American's women participation in breast cancer screening programs, could impede nursing's progress in improving access to breast health care.

Research is used to generate and test theory (Chinn \& Kramer, 1995), and existing research on access has generated evidence of additional delineators of access to breast health care which should be examined. This study provides an opportunity to examine the relationship between other aspects of access and utilization of breast health care services within an "equal" economic access health care delivery system; and to provide a knowledge base for the formation of hypotheses to be tested in future breast health care research focused on women of color. 


\section{Definition of Terms}

Economic access: the availability of health care and health insurance coverage (Aday, Andersen, Fleming, 1980), as indicated by a woman's eligibility to receive care in the MHS being indicated on the Tumor Registry medical record.

Potential access:

Age: the age of the woman at the time of diagnosis as indicated on the Tumor Registry medical record.

Race: the classification of an individual based on physical characteristics.

1. Black woman is defined as any woman identified as non-Hispanic black or African American on the Tumor Registry medical record.

2. White woman is defined as any woman identified as non-Hispanic White or Caucasian on the Tumor Registry medical record.

Socioeconomic Status (SES): the active duty rank of the woman or military sponsor entitling the woman to receive health care within the MHS (Clark \& Thompson, 1994), as indicated on the Tumor Registry medical record.

1. Enlisted ranks are defined as E1 (enlisted at the first level of rank) through E9 (enlisted at the ninth level of rank).

2. Officer ranks are defined as Chief Warrant Officer (CWO) and O1 (commissioned officer at the first level of rank) through $\mathrm{O} 10$ (commissioned officer at the tenth level of rank).

3. Junior Enlisted ranks are defined as E1 through E5.

4. Senior Enlisted ranks are defined as E6 through E9. 
5. Junior Officer ranks are defined as $\mathrm{CWO}$ through $\mathrm{O} 4$.

6. Senior Officer ranks are defined as O5 through $\mathrm{OlO}$.

Means of discovery of breast cancer: The means of discovery of a breast lump, mass, or abnormality that initiated the woman's utilization of specialized breast health services and resulted in the diagnosis of breast cancer. Indicated on the Tumor Registry medical record as one of the following means:

1. Intentional breast self exam (BSE): discovered by the woman while routinely examining her own breasts.

2. Incidental BSE: incidentally or accidentally discovered by the woman or significant other.

3. Routine clinical breast exam (CBE): discovered by a provider of health care within the MHS or other health care delivery system during a routine breast health care exam.

4. Secondary CBE: discovered by a provider of health care within the MHS or other health care delivery system during a physical exam for purposes other than routine breast health care.

5. Routine mammogram: discovered during performance of routine clinical mammogram.

6. Secondary mammogram: discovered during a clinical mammogram performed as a result of a complaint of a medical condition other than breast related. Realized access:

Utilization of services measured by: 
Length of time between discovery and diagnosis - the length of time in days between discovery of breast lump, mass, or abnormality and diagnosis of breast cancer, as indicated on the Tumor Registry medical record.

Length of time between diagnosis and treatment - the length of time in days between diagnosis of breast cancer and initiation of treatment of breast cancer (excision of lump, mastectomy, chemotherapy, or radiation therapy), as indicated on the Tumor Registry medical record.

Stage at diagnosis of breast cancer: refers to the grouping of patients according to the extent of their disease as indicated on their Tumor Registry medical record. This grouping is defined in accordance with the classifications outlined for clinical staging of breast cancer in the American Joint Committee On Cancer (AJCC) Cancer Staging Manual (1997). Staging is based on classification of the primary tumor $(T)$, regional lymph nodes $(N)$, and distant metastasis $(M)$. A summary of these classifications is presented in tables 1,2 , and 3 . Table 4 summarizes the clinical staging of breast cancer at diagnosis, using the TNM classifications.

Stage classifications for this study are defined as:

1. In situ: Stage 0

2. Stage 1: Stage I

3. Stage 2: $\quad$ Stages IIA and IIB

4. Stage 3: Stages IIIA and IIIB

5. Stage 4: Stage IV

6. Early stage: In situ and stage 1; Late stage: stages 2, 3, and 4. 
Table 1

AJCC Primary Tumor ( $T$ ) Classification

\begin{tabular}{|l|l|}
\hline TX & Primary tumor cannot be assessed \\
\hline T0 & No evidence of primary tumor \\
\hline Tis & Carcinoma in situ \\
\hline T1 & Tumor $2 \mathrm{~cm}$ or less in greatest dimension \\
\hline T2 & Tumor more than $2 \mathrm{~cm}$ but not more than $5 \mathrm{~cm}$ in greatest dimension \\
\hline T3 & Tumor more than $5 \mathrm{~cm}$ in greatest dimension \\
\hline T4 & Tumor of any size with direct extension to chest wall or skin. \\
\hline
\end{tabular}

Table $2 \quad$ AJCC Lymph Nodes $(N)$ Classification

\begin{tabular}{|l|l|}
\hline NX & Regional lymph nodes cannot be assessed \\
\hline N0 & No regional lymph node metastasis \\
\hline N1 & Metastasis to moveable ipsilateral axillary lymph node(s) \\
\hline N2 & Metastasis to ipsilateral axillary lymph node(s) fixed to one another or to other structures \\
\hline N3 & Metastasis to ipsilateral internal mammary lymph node(s) \\
\hline
\end{tabular}

Table $3 \quad$ AJCC Distant Metastasis (M) Classification

\begin{tabular}{|l|l|}
\hline MX & Distant metastasis cannot be assessed \\
\hline M0 & No distant metastasis \\
\hline M1 & Distant metastasis \\
\hline
\end{tabular}


Table $4 \quad$ AJCC Clinical Staging of Breast Cancer

\begin{tabular}{|c|c|c|c|}
\hline Stage & Primary Tumor & Regional Lymph Nodes & Distant Metastasis \\
\hline Stage 0 & Tis & N0 & M0 \\
\hline Stage I & T1 & N0 & M0 \\
\hline Stage IIA & T0 & N1 & M0 \\
& T1 & N1 & M0 \\
& T2 & N0 & M0 \\
\hline Stage IIB & T2 & N1 & M0 \\
& T3 & N0 & M0 \\
\hline Stage IIIA & T0 & N2 & M0 \\
& T1 & N2 & M0 \\
& T2 & N2 & M0 \\
& T3 & N1 & M0 \\
& T3 & N2 & M0 \\
& T4 & Any N & M0 \\
\hline Stage IV & Any T & N3 & M0 \\
\hline & Any T & & M1 \\
\hline
\end{tabular}




\section{Assumptions}

The assumptions on which this study was based include:

1. All information contained in the Tumor Registry medical record is accurate and reflects information included in the woman's hospital medical record.

2. Health care resources within the MHS are adequate in terms of the availability of health care personnel, equipment, and educational services.

3. All women entitled to receive health care within the MHS have "equal" economic access to breast health care services. 


\section{CHAPTER II}

\section{REVIEW OF THE LITERATURE}

The literature pertaining to breast cancer mortality disparity between African American and Caucasian women was critically reviewed for its relevance to this study. This chapter presents a review of research studies and findings that support the relationship between the study variables: economic access, age, race, SES, the means of discovery of breast cancer, delay in diagnosis of breast cancer, delay in treatment of breast cancer, and stage at diagnosis of breast cancer. Studies included in this review tend to address multiple aspects of this study's variables. For instance, age, race, and SES are included as demographic variables in many of the studies reviewed, and the means of discovery of breast cancer (BSE, CBE, mammogram) is frequently included as a variable in studies examining delay in diagnosis and treatment of breast cancer. Therefore, for organizational purposes, the following subsections are arranged based on the major variable examined in the research study under review, as it relates to stage at diagnosis of breast cancer, and breast cancer mortality disparity between African American and Caucasian women.

\section{Economic Access and Stage at Diagnosis of Breast Cancer}

Studies exploring access as a barrier to cancer care and screening have defined access primarily in terms of economic access and in relation to health care insurance coverage. Many of these studies have focused on access to care created by federally, 
state, and locally financed health care programs, resulting from implementation of health care policy. "Improved access to care is an important goal of much of health policy" (Aday \& Andersen, 1974, p. 212). However, barriers to access may occur at the health care delivery system level or at a personal level; and an individual's ability and willingness to participate in health care determines access, as well as the economic availability of health care (Aday, et al., 1993; Bashshur, Homan, Smith, 1994; DeBaca, 1994; Facione, Dodd, Holzemer, \& Meleis, 1997; Facione \& Facione, 1997; Friedman, 1994; Guidry, Aday, Zhang, \& Winn, 1997; Guidry, et al., 1996; Katz, Hislop, Thomas, \& Larson, 1993; Lauver \& Ho, 1993; Lierman, 1988; Northam, 1996; Powell, Schwartz, \& Hussain, 1995). In this study, economic access to health care was controlled for by examining participation in breast health care by women eligible to receive care within the MHS. The studies included in this section of the review examine the relationship between health insurance coverage, demographic characteristics of the individual seeking care, and stage at diagnosis of breast cancer.

In examining the relationship between health insurance coverage and clinical outcomes among women with breast cancer, Ayanian, Kohler, Abe, and Epstein (1993) studied the state cancer registry records of 4675 women, 35 to 64 years of age, diagnosed with breast cancer in New Jersey between 1985 and 1987. The findings of this study reported that 88.3 percent of the women with private insurance were White, 70.8 percent of those uninsured were White, and 64.4 percent of those with Medicaid were White. The researchers compared the stage of breast cancer at diagnosis among uninsured and Medicaid patients with the stage at diagnosis of disease among privately insured patients 
and found that uninsured women $(\mathrm{P}<0.001)$ and women covered by Medicaid $(\mathrm{P}=0.01)$ presented with significantly more advanced disease than those women who were privately insured. Stratification of the population according to age revealed that significantly more advanced disease was present among uninsured women 50 to 64 years of age $(P<0.001)$ and those covered by Medicaid who were 35 to 49 years of age $(P=0.02)$, than among those women with private health insurance coverage, in the same age groups. An evaluation of stage specific survival by type of insurance coverage found that survival was worse for uninsured $(\mathrm{P}<0.001)$ and Medicaid $(\mathrm{P}<0.001)$ patients with local disease, than for privately insured patients. Survival was also worse for uninsured $(P<0.001)$ and Medicaid $(\mathbf{P}<0.001)$ patients with regional disease than for privately insured patients, but there was no difference among the comparison groups for distant disease. The results of this study support that women without health care insurance and with Medicaid coverage presented with more advanced disease than did women with private health insurance. After using multivariate analysis to estimate the adjusted risk of death among uninsured and Medicaid patients, when compared to those who were privately insured, the researchers found that uninsured and Medicaid patients had a greater adjusted risk of death. In addition, those women who were uninsured or with Medicaid, had poorer survival with local and regional disease than did those with private health care insurance; and association between survival and type of insurance coverage was identified.

Chaulk, Kazandjian, and Pipesh (1995) conducted a retrospective analysis using hospital discharge data from 1989 through 1993 to explore racial differences in breast cancer illness, comorbidity, and mortality in Maryland's unique hospital all-payer rate 
setting system. The all-payer rate setting system has been shown to constrain economic incentives in the decision to hospitalize and provide services once hospitalized. In a sample of 14,016 women (11,231 White, 2487 Black, and 298 other racial origin), the researchers found that despite the presence of the all payer system, Black women hospitalized for breast cancer were more likely to be severely ill than white women. The findings of the study also demonstrated that more Black women died $(2.3 \%$ or 58 of 2487 ) between 1989 and 1993, than did White women $(1.4 \%$ or 161 of 11,231$)$. In addition, a relationship between the type of insurance coverage and the severity of the illness was also found to be present and was irrespective of race. The percent of women insured by Medicare was $44.9,44.5$ percent had private insurance, 4.3 percent were enrolled in a Health Maintenance Organization (HMO), and 2.5 percent were uninsured. Medicaid recipients were significantly more ill than Medicare enrollees, privately insured women, or women insured by an HMO. The researchers did not control for education, care seeking behavior, or geographic access to services, and the discharge data reviewed for the study did not indicate whether or when breast cancer screening was performed on the women in the study. This study also demonstrated a relationship between the type of health care insurance coverage and stage at diagnosis of disease.

Mandelblatt, Andrews, Kao, Wallace and Kerner (1995) used data from the New York state department of health tumor registry to examine the impact of demographic variable such as age, race, ethnicity, and type of reporting hospital; and social context variables such as area of residence, socioeconomic status and access to screening mammography, on breast cancer stage at diagnosis among New York women between 
1980 and 1985. Chi-square and t-tests were used to assess the relationship between the demographic variables and each stage. Late stage (stages 2,3, and 4) breast cancer was found to occur at a significantly higher proportion among women 50 to 64 years of age (54.7\%) than among women 20 to 49 years of age (50.9\%) and women 65 and older $(50.5 \%)(\mathrm{P}<0.0001)$. Late stage disease occurred at the highest proportion in African American women (59.2\%), followed by Hispanic women (54.6\%), and White women $(50.4 \%)(\mathrm{P}<0.001)$. Women diagnosed in public hospitals had a higher proportion of late stage disease (63.7\%) than did women diagnosed in nonpublic hospitals $(51.1 \%)$. Three logistic regression models were used to evaluate the impact of demographic variables, social context variables, and both class of variables combined, on the outcome of having breast cancer diagnosed in a late stage, compared to an early stage. Women diagnosed in public hospitals were 45 percent more likely to be diagnosed at a later stage than women diagnosed in other types of hospitals $(\mathbf{P}<0.0001)$; and African American women were 34 percent more likely to be diagnosed at a later stage than White women $(\mathrm{P}<0.0001)$. The odds of having late stage disease were 28 percent higher for women living in areas with the lowest socioeconomic status ranking, when compared with women living in an area of the highest socioeconomic status ranking. Mammography capacity was a significant independent predictor of stage at diagnosis of breast cancer $(P=0.016)$.

Zaloznik (1995) conducted a retrospective review of DoD MHS breast cancer cases recorded in the DoD Automated Tumor Registry (ACTUR), from 1976 to 1992, to determine if there were any differences in stage at diagnosis of breast cancer or survival between Caucasian and African American women being diagnosed in the MHS. Zaloznik 
examined a total of $6414(89.58 \%$ of total) records of Caucasians and $746(10.41 \%$ of total) records of African Americans. The subjects were stratified according to age at diagnosis, race, and stage at diagnosis, and the researcher found that there were no statistically significant differences in stage at presentation between Caucasian and African American women. Caucasian women had significantly $(\mathrm{P}=<0.0001)$ more tumors $\leq 1.0$ centimeters than did African American women. African American women had more tumors that measured 2.1 to 3.0 centimeters in size. The median age for diagnosis of breast cancer in African American women was 50, as compared to age 58 in Caucasian women. Twenty-four percent of the African American women studied were less than 40 years old at presentation, compared to nine percent of the Caucasian women $(\mathrm{P}=<$ 0.0001 ). Even though the findings of this study did not report a significant difference in the stage at diagnosis of breast cancer between African American and Caucasian women, significant differences were reported in tumor size and age at diagnosis. This study did not evaluate the effect of socioeconomic status or geographic location of the treatment facility on stage at diagnosis, nor did it look at care seeking or screening behaviors.

Wojcik, Spinks, and Optenberg (1998) conducted a retrospective review of 6577 DoD MHS central tumor registry records to evaluate the effects of age, stage of cancer at diagnosis, tumor size, grade, lymph node involvement, waiting time between diagnosis and first treatment, marital status, military dependent status, alcohol usage, tobacco usage, and family history on breast cancer survival. The 5879 White ( $89.4 \%$ of the total) and 698 African American (10.6\% of the total) women included in the study ranged in age from 19 to 97 years, and were diagnosed with breast cancer between 1975 and 1994 . 
Chi-square analysis and t-tests were used to compare the characteristics of the African American and White women. The Cox proportional hazards model and several multiple regression models were applied to examine the effects of the independent variables on survival status. Almost 90 percent of the women in the study were civilian spouses of active duty service members or military retirees, and 4.9 percent of the women were active duty members themselves. Within the group of African American women, 10.3 percent were on active duty when diagnosed with breast cancer as compared to 4.3 percent within the group of White women. The mean age at the time of diagnosis for African American women was 50 years as compared to 57.1 years for the White women; and 22.8 percent of the African American women were diagnosed with breast cancer at less than 40 years of age, as compared to 9.2 percent of the White women. Wojcik, et al. found statistically significant $(p=0.0001)$ differences in overall survival between the African American and White women. The mortality rate among African American women was 24.77 percent as compared with 18.08 percent for White women. Tumor stage at the time of diagnosis appeared to be the strongest predictor of death from all causes. The risk of death for women with tumors diagnosed at stage 3 was 6.55 times higher than for women diagnosed at stage 0 . African American women had significantly larger tumors than White women ( 2.53 centimeters as compared to 2.28 centimeters, $p=0.013$ ); and 48.86 percent of the African American women were diagnosed at stages 2 and 3 as compared to 38.65 percent of the White women. The waiting time from diagnosis to treatment was significantly shorter for African American women (21.15 days) than for White women (29.51 days; $p=0.0024)$. 
Despite the significant difference in survival status between African American and White women within the DoD MHS, Wojcik, et al. found that the five year risk of death for African American women receiving care in the DoD (1.37), when compared with White women, was less than the relative risk reported in the Surveillance, Epidemiology, and End Results (SEER) Program of the National Cancer Institute (1.86). The survival rate for White women receiving care within the DoD was comparable to rates reported in SEER. Based on these study findings, the researchers surmised that ready access to medical facilities improves survival for African American women, and support that even though ready access is necessary for receipt of definitive medical care, it is not a sufficient condition for equalizing survival rates between African American and Caucasian women. Wojcik, et al. did not examine the effects of socioeconomic status or means of discovery on stage at diagnosis or survival status, and suggest that other explanations may contribute to diagnosis of African American women at younger ages and later stages of breast cancer.

The studies presented above support the existence of a relationship between economic access and stage at diagnosis of breast cancer. The studies also identify differences in stage at diagnosis of breast cancer relative to the type of health care insurance coverage, irrespective to race. Even when access to care was provided though health insurance or public policy as a result of local, state, or federal health care funding, diagnosis delay occurred. Existence of economic access to health care did not always result in early diagnosis of breast cancer, and other factors such as age, race, and SES were found to influence breast cancer diagnosis outcomes. 
Even though the studies presented above examined the impact of age, race, and SES on stage at diagnosis of breast cancer, and the type of health care insurance being utilized, the studies did not address the influence of the means of discovery of breast cancer (BSE, CBE, mammogram), or delays in diagnosis of breast cancer, on stage at diagnosis in women with economic access to health care.

\section{Potential Access and Stage at Diagnosis of Breast Cancer}

The availability and organization of resources within a health care delivery system, and the characteristics of the desires, needs, and resources that a potential consumer brings to the care seeking process, influence the probability of an individual's entry into the health care system (Aday, Fleming, \& Andersen, 1984; Andersen, Giachello, \& Aday, 1986; Berk \& Schur, 1998; Guidry, et al., 1996). Therefore, potential access is characterized by: predisposing factors such as age, sex, race, religion, and values concerning health and illness; enabling factors such as income, insurance coverage, and attributes of the community in which the individual lives; and need factors such as illness level and the need for health care services as perceived by the individual or health care delivery system (Aday \& Andersen, 1974; Aday, Andersen, \& Fleming, 1980). Because the setting for this study was the MHS, the assumption was made that health insurance coverage was available and equal for all eligible military beneficiaries. Nevertheless, factors such as age, race, SES, and the event initiating breast health care differ among individuals receiving care in the MHS, and may cause varying levels of potential access within an equal access system. Therefore, the following section presents a review of the 
literature of the relationship between variables of potential access identified for inclusion in this study, and stage at diagnosis of breast cancer.

\section{Age}

The single most important risk factor for breast cancer in women is age, although race and ethnicity modify the effect of age on the risk of developing breast cancer (Vogel, 1996). The purpose of this review, however, is to explore the relationship between age and stage at diagnosis of breast cancer. Age is represented as a demographic variable in most of the studies presented in this chapter. Therefore, this section of the literature review will focus on studies in which age was the major variable of the study in examining its relationship to stage at diagnosis of breast cancer.

Newman and Alfonso (1997) evaluated stage at diagnosis of breast cancer distribution, relative to age, in women treated for breast cancer between 1990 and 1995 at the Long Island College Hospital in Brooklyn, New York. For the purposes of this study, the researchers conducted a retrospective review of tumor registry data, hospital and physician office records, and pathology reports on 283 White patients and 143 Black patients. The two groups of women were stratified according to age and women 50 years and older were designated as the older patient population, and women younger than 50 years were designated as the younger population. SES was estimated by access to primary care management through the outpatient public clinic as compared to access to private medical care. Chi-square analysis was used to determine statistical significance. Newman and Alfonso found that the mean age at diagnosis for White women was 63 years compared to 56 years for Black women; and that approximately one fifth of the White 
women were diagnosed at less than 50 years of age compared to one third of the Black women $(\mathrm{P}=0.008)$. Black women were diagnosed with in situ breast cancer in 8.6 percent of the cases, and stage 1 cancer in 23.7 percent of the cases, while White women were diagnosed with in situ breast cancer in 10.4 percent of the cases and stage 1 breast cancer in 37.2 percent of the cases $(P=0.004)$. Stage 3 and 4 cancers were diagnosed at a significantly higher rate in Black women (27.3 percent) when compared to White women (18.6 percent $)(\mathrm{P}=0.049)$.

Stage 2,3 , and 4 breast cancer was diagnosed at a proportion of 64.6 percent in the younger population of women as compared to 55.8 percent in the older population. There were no notable differences in the distribution of stage at diagnosis of breast cancer in the older patient population. In the younger population, in situ breast cancer was diagnosed in 22 percent of the Black women as compared to 6.4 percent of the White women; and stage 0 and 1 breast cancer was diagnosed at a significantly lower rate in Black women at 21.3 percent, when compared to 49.0 percent in White women $(\mathrm{P}=$ 0.004). Stage 3 disease occurred at the highest proportion in the younger Black women. Stage 0 and 1 breast cancers occurred at a proportion of 38.1 percent in younger Black women as compared to 21.3 percent in older Black women $(\mathrm{P}=0.049)$.

Black women were diagnosed through the hospital's public health clinic in 9.7 percent ( 14 of 144) of the cases as compared to 1.3 percent ( 3 of 239) of the White women, but over 90 percent of both groups of women had access through the private practice setting. The stage at diagnosis of breast cancer distribution for Black women of all ages was similar among the public clinic patients and private practice setting patients. 
Stage 0 breast cancer was diagnosed in 9.7 percent of the Black women receiving care in the private setting, zero percent was diagnosed in women receiving care in the public setting. Stage 1 and 2 breast cancers were diagnosed in 63.7 percent of the Black women receiving care in the private setting as compared to 64.3 percent in the public setting. Stage 3 and 4 cancers were diagnosed in 26.6 percent of the Black women receiving care in the private setting as compared to 35.7 percent in the public setting.

The study presented above confirms that there is a higher incidence of late stage breast cancer diagnosed in Black women when compared to White women. The study also confirms that late stage breast cancer is diagnosed at a higher rate in younger Black women when compared to older Black women and White women of all ages. However, these race related variances do not appear to be associated with the type of health care coverage since the majority of the Black women in the study had access to and used private medical care; and the distribution of stage at diagnosis of breast cancer in Black women receiving care through the public clinic was similar to the distribution of those receiving care through the private practice setting. This study did not examine the influence of care seeking behaviors on stage at diagnosis of breast cancer.

\section{$\underline{\text { Race }}$}

Most of the arguments for biological contributions to the disparity in breast cancer death rates are based on race. However, there is growing consensus in the health care literature to support that human races are not biological entities, and that racial classification in the United States is determined by social and political parameters (Hahn \& Stroup, 1994; Herman, 1996; Roach \& Alexander, 1995). In addition, the concept of race 
has been purported to be socially meaningful, but of limited biological significance; and the factors creating the breast cancer mortality disparity have been argued to be not as much biological as they are cultural, social, geographical, and structural (Adams, 1995; BayneSmith, 1996; Frank-Stromborg \& Olseri, 1993; Freeman, 1997; Kato \& Mann, 1996; Williams, Lauizzo-Mourey, \& Warren, 1994). The studies presented in the following section integrate examination of the relationship between cultural, social, structural, and biological perspectives of race, and stage at diagnosis of breast cancer in African American and Caucasian women.

Coates et al. (1990) examined the effects of nutritional status on breast cancer survival differences among 469 Black and 1491 White women diagnosed with breast cancer in Georgia between 1975 and 1979. Nutritional status variables included height measured in meters, weight measured in kilograms, and serum albumin, hemoglobin, and lymphocyte counts. Demographic, medical, and clinical characteristics such as: age in years, marital status, education in years, menopausal status at diagnosis, prior history of cancer, family history of cancer, history of breast self examination, number of lymp nodes involved, type of treatment (surgery, radiation therapy, hormone therapy, chemotherapy), occupational status of the patient's household, stage at diagnosis of breast cancer, tumor histology, time between the recognition of symptoms and the first medical consultation, and time between the first medical consultation and diagnosis were also included in the analysis. This study employed several statistical tests. Stage and race specific median body mass indices, lymphocyte counts, and levels of serum albumin and hemoglobin were compared to assess associations between race and nutritional status indicators. Odds 
ratios and corresponding approximate 95 percent confidence intervals were used to evaluate the associations between race and the nutritional status indicators, and between race and other suspected prognostic factors identified through the research literature. Univariate survival analyses, with the Kaplan-Meier product estimation procedure, were performed to assess the relationship between suspected prognostic factors and survival from breast cancer. The Wilcoxon test statistic was used to assess statistical significance of differences between survival curves. The prognostic effect of race was evaluated using the Cox proportional hazards regression approach with adjustments made for nutritional status variables and other confounding prognostic factors.

Coates et al. found that Black women in this study were more likely to be younger, unmarried, less educated, and of a low occupational status than White women. Black women were less likely to have a personal history of breast cancer than were White women; and Black women were less likely to perform BSE than were White women. Black women tended to have a longer time between recognition of symptoms and seeking medical care; and Black women tended to have more advanced disease and lymph node involvement, and were more likely to present with nipple bleeding and ulceration, at the time of diagnosis, than were White women. Because Black women were more likely to present with advanced, inoperable disease, when compared to White women, the percentage of Black women not undergoing surgery was elevated. When data were adjusted for stage at diagnosis of breast cancer, socioeconomic status, and other prognostic factors mentioned above, poorer survival rates were demonstrated in Black women when compared to White women. There was a strong association between race 
and three of the four nutritional status measures. Lower levels of serum albumin and hemoglobin and higher relative body weight were more common among Black women than among White women, and were independently associated with poorer survival. Approximately 76 percent of the White women in the study survived five years as compared to 57 percent of the Black women. The black-to-white hazard rate ratio among women with stage 3 disease was 1.61. Adjustment for demographic, medical, and clinical characteristics among women with stage 3 disease reduced the black-white difference in mortality rates in these women to a ratio rate of 1.54 . However, simultaneous adjustment for all four of the nutritional status variables reduced the rate ratio to 1.14 , resulting in a 74 percent reduction in excess risk for Black women.

The results of this study support that differences in the nutritional status of Black and White women may contribute to differences in survival. Black women were more likely to have lower serum albumin and hemoglobin levels than White women, and were more likely to be overweight. These differences were associated with poorer survival rates, and in women with stage 3 disease, adjustment for these nutritional status indicators explained three fourths of the excess risk of mortality among the Black women in this study. Serum albumin, commonly used as an indicator of nutritional status, reflects protein calorie nutrition intake. However, serum albumin is also a reflection of disease status in various illnesses (Coates et al., 1990). The researchers in this study acknowledged that the lower serum albumin levels in the Black women in this study could be a reflection of nutritional status or a reflection of the extent of the disease within stage. Decreased serum hemoglobin levels can reflect malnutrition or may result from the 
presence of disease, including breast cancer. Therefore, the associations found between decreased serum albumin and hemoglobin levels could be indicative of either nutritional status or disease severity. This study's findings about the poorer survival rates among overweight Black women with breast cancer are consistent with other research studies (Coates et al., 1990); however, it is not clear if the association noted between obesity and stage at diagnosis is a reflection of masking of signs and symptoms of breast cancer due to being overweight or hormonal mechanisms.

Jones, Kasi, Curnen, Owens, and Dubrow (1997) also evaluated the effect of obesity on poorer stage at diagnosis outcomes for Black women when compared to White women. In conducting a retrospective review of 145 Black women and 177 White women diagnosed with breast cancer in Connecticut between January 1987 and March 1989, these researchers examined the extent to which the observed racial differences in stage at diagnosis of breast cancer could be explained by racial differences in severe obesity. Odds ratios and their 95 percent confidence interval and logistic regression were used to test for significant associations between race, obesity, and stage at diagnosis. Jones et al. found that severe obesity was associated with both race and stage at diagnosis of breast cancer. Severe obesity was more likely to occur in Black women (26 percent) than in White women ( 7 percent); and severe obesity was significantly associated with diagnosis of breast cancer in stage 2 or greater. After adjustment for severe obesity, the risk of diagnosis of breast cancer at a later stage was reduced by 33 percent in Black women as compared to White women. The findings of this study concluded that severe obesity may 
play an important role in explaining the higher prevalence of late stage diagnosis of breast cancer in Black women.

Hunter et al. (1993) obtained data from the National Cancer Institute's Black/White Survival Study for the period 1985 to 1986 and evaluated the relationship between selected demographic, lifestyle, health care access, antecedent medical experience factors, and stage at diagnosis of breast cancer in 649 Black and 573 White women. The women included in the study were newly diagnosed with primary breast cancer between January 1, 1985 and December 31, 1986; ranged in age from 20 to 79; and resided in one of the following areas: Atlanta, Georgia; New Orleans, Louisiana; or the San FranciscoOakland, California area. Approximately 70 percent of the Black breast cancer cases were randomly selected from each metropolitan area, and a sample of White breast cancer cases was matched by age groups to adjust for the tendency for the Black women to be younger than the White women at the time of diagnosis. Adjustment of the patient selection rates resulted in a slight imbalance in the race distribution in the study, 53 percent Black women and 47 percent White women. Hunter et al. collected data from medical records, review of histology slides, and patient interviews. Demographic factors included marital status, education, and total family income. Lifestyle factors included smoking history and body mass index. Antecedent medical experience factors included history of BSE and CBE during the six years prior to breast cancer diagnosis; length of time from the woman's recognition of a symptom to the first visit to a physician for that symptom; and current medical conditions that the women had at the time of admission to the hospital for a diagnosis of breast cancer. Access to medical care factors included the 
type of health insurance and usual source of medical care. Stage at diagnosis was classified according to the tumor-lymph node-metastases (TNM) system for breast cancer based on post surgical pathologic evaluation criteria of the American Joint Committee on Cancer (Stages: in situ, I, II, III, and IV).

Hunter et al. evaluated the differences in breast cancer stage at diagnosis and the distribution of the factors mentioned above, between the Black and White women included in this study by using chi-square tests for independence, and chi-square test for trend, where appropriate. Log-linear models were used to evaluate the relationship between each factor and stage at diagnosis separately in the groups of Black and White women. Logistic regression models were used to examine explanatory factors that showed a statistically significant association with stage at diagnosis in both groups of women. The researchers found a highly significant association between race and stage at diagnosis $(\mathrm{P}<.00005)$. Black women had a more advanced stage of disease at diagnosis. There were no significant differences in age adjusted race-stage relationship by metropolitan area. Women younger than 50 years old with in situ disease were significantly more likely to be White, while those with stage III or IV were significantly more likely to be Black. There was no statistically significant difference in stage at diagnosis observed in women 65 to 79 years of age. Black women were more likely than White women $(\mathrm{P}<.001)$ to have less than a high school education, be overweight, and have a lower total family income. Black women were also more frequently never married and more often current smokers, and had a greater history of existing medical conditions. In addition, a greater proportion of Black women, than White women, were found to 
either have no insurance or have public assisted health care, with their usual source of medical care being the hospital outpatient clinic, emergency room, or public clinic. The majority of the women in both groups reported practicing BSE and participating in CBE; and the length of time from recognition of symptoms to seeking medical consultation was equal in both races. A recent history of CBE was significantly associated with lower stage disease in both groups (Black women, $\mathrm{P}<.01$; White women, $\mathrm{P}<.04$ ); and a history of delay between recognition of symptoms and medical consultation was positively related to a higher stage at diagnosis in both races $(\mathrm{P}<.00005)$. Limited access to health care, usual source of medical care, larger body mass index, and history of low mammography use factors were associated with a higher stage at diagnosis of breast cancer only in the Black women in the study. Lower income was associated marginally with higher stage of disease only in the White women in the study $(P=.06)$; and less education was associated marginally with higher stage of disease at diagnosis only in the Black women in the study $(\mathrm{P}=.07)$

The Black women in this study were diagnosed at a higher stage of disease than the White women. In the Black women, access to health care, lifestyle, and antecedent medical experience were factors found to be associated with higher stage at diagnosis; and in the White women in this study, SES tended to have more influence on higher stage at diagnosis of breast cancer. Women with higher total family income, and medical care through private physicians or private medical clinics were found to present with lower stage of disease, suggesting that higher stage at diagnosis is related to poorer access to health care and lower SES. However, this association is social, and perhaps 
environmental, but there are no data in this study to support that it is biological. The racestage association observed in this study is complex and supports that there are multiple factors influencing differences between stage at diagnosis in the Black and White women included in this study. Hunter et al. evaluated factors associated with race, age, SES, the means of discovery of breast cancer, and treatment and diagnosis delay. However, the evaluation was conducted across three metropolitan areas with varying social, political, and environmental climates; and in access systems that have the potential to vary in health care availability, quality, and promptness. Even though this study is systematic and comprehensive in its evaluation of factors associated with differences in stage at diagnosis of breast cancer between Black and White women, studies systematically evaluating the influence of these factors on stage at diagnosis of breast cancer in an equal economic access system are needed.

Eley et al. (1994) used data from the National Cancer Institute's Black/White Cancer Survival Study for the period of 1985 to 1986 to examine the impact of recognized prognostic factors on the observed poorer breast cancer survival rate in Black women when compared to White women. The study included 612 Black and 518 White women, age 20 to 79 from three different geographic areas. The sampling design was based on random sampling of Black subjects and frequency matching of White subjects by age groups. Data were derived from personal interviews with the women, abstracts of the hospital records, and a central pathology review of biology and surgical specimens in exploring the impact of stage at diagnosis, histopathologic characteristics of the tumor, treatment, concurrent medical conditions, socioeconomic status, and usual source of care, 
on survival differences. Frequency tables and chi-square test were used to examine bivariate relationships between race and the variables mentioned above. Cox proportional hazards regression was used to asses the impact of the variables mentioned above on racial differences in survival after diagnosis of breast cancer. After controlling for age and geographic area, Eley et al. found that the risk of dying from breast cancer was 2.2 times greater for Black women than for White women and concluded that approximately 75 percent of the racial difference in survival was explained by the prognostic factors explored in the study (stage at diagnosis, histopathologic characteristics of the tumor, treatment, concurrent medical conditions, SES, usual source of medical care, marital status, occupation, poverty index). Approximately 40 percent of the difference in the survival rate was explained by a more advanced stage of the disease in the Black women. After controlling for stage and tumor pathology, information about treatment did not explain the survival difference. Racial differences in stage at diagnosis of breast cancer appeared to be largely influenced by social and demographic factors.

Simon and Severson (1996) examined racial differences in survival of breast cancer in 8622 White and 1880 African American women diagnosed with breast cancer in the Detroit metropolitan area between 1988 and 1992 . This retrospective analysis was conducted using medical and demographic data obtained from the Metropolitan Detroit Cancer Surveillance System. Demographic data included age at diagnosis, race, marital status at diagnosis, and census derived socioeconomic rank. Clinical variables included tumor size, stage at diagnosis, histologic type and histologic grade. Factors relating to the characteristics of the hospital in which each woman received their first course of cancer 
related surgery included the number of beds in the hospital, presence of a residency training program, and the proportion of Medicaid and/or Medicare patients discharged from each hospital. The chi-square test was used to assess differences between age, marital status, socioeconomic census rank, tumor size, stage at diagnosis, histologic type and grade of the tumor, number of beds in the hospital, presence of residency training, proportion of Medicaid and/or Medicare discharges, and race. The relative risk of death from any cause in African American women, when compared to White women, was estimated using Cox proportional hazards regression.

Simon and Severson found that the African American women in this study were more likely to be younger than the White women, when diagnosed with breast cancer. In addition, African American women were more likely to live in a census tract with a lower socioeconomic census rank than were White women. African American women were found to present at a more advanced stage, with larger tumors, and a higher tumor histologic grade. When examining local (confined to the breast) and regional (spread beyond the breast by direct extension or to regional lymph nodes) stage disease, the researchers found that White women had a significantly better survival than did African American women (log rank, $P=0.0001)$. Although not significantly different within remote (direct extension or metastases to organs other than regional or distant lymph nodes) stage disease, White women seemed to have better survival rates than did African American women $(\log$ rank, $P=0.3)$. There was a significant difference in survival between African American women and White women in the first four years after diagnosis, however there was no significant difference in women who lived longer than 
four years. There was a significant interaction between age at diagnosis and race $(\mathrm{P}=$ 0.005). Fifty percent of the variation in survival between African American and White women was accounted for by adjusting for age, marital status, socioeconomic census rank, stage at diagnosis, characteristics of the tumor, number of beds in the hospital of treatment, presence of residency training, and the proportion of Medicaid and/or Medicare discharges.

Roach et al. (1997) evaluated the prognostic significance of race and survival in stage 2 breast cancer in 185 African American, 1325 White, 12 Oriental, and 34 other [race] women treated with adjuvant chemotherapy in the Cancer and Leukemia Group B (CALGB) trial 8541. Retrospective analysis was conducted using data from the CALGB database. Women included in the CALGB trial had: (a) Histologically confirmed stage 2 breast cancer with no or minimal symptoms; (b) clear margins following radical, modified, or breast conserving surgery; (c) chemotherapy preceded by dissection of level one and two lymph nodes; (d) chemotherapy followed by external beam irradiation consisting of 50 Gy to the entire breast and 1.5 Gy boost to the area of incision; (e) initial chemotherapy including CAF (cyclophosphamide, doxorubicin, fluorouracil); (f) tamoxifen added in women who were perimenopausal, or who were postmenopausal with positive estrogen receptors; and (g) chemotherapy prescribed based on actual body weight. Women included in the CALGB 8541 trial were randomly assigned to receive either a high dose, standard dose, or low dose intensity of chemotherapy; and were stratified according to type of breast management, menopausal status, number of lymph nodes involved, and estrogen receptor status. 
In this study, differences in disease free survival and overall survival were assessed. "Disease free survival was defined as the time from randomization to a documented relapse or death without a relapse. Overall survival was defined as the time from randomization to death from any cause" (Roach et al., 1997, p 108). The proportional hazards model for multivariate analyses, the log rank test, and the stratified log rank test for univariate analyses were used to assess differences in disease free and overall survival.

African American women were found to be slightly more likely to be estrogen receptor positive (34 percent) than were White women ( 26 percent) $(P=0.03)$. Ninety percent of the African American women were more likely to have had a mastectomy when compared to White women (84 percent) $(\mathbf{P}=0.03)$. African American women tended to be younger (median 46 years) than White women (median 50 years) $(\mathrm{P}<0.01$ ); and African American women tended to have tumors somewhat larger (median 3.0 centimeters) than White women (median 2.5 centimeters) $(\mathrm{P}<0.01)$. When compared to White women, African American women tended to have more delays in treatment $\mathrm{P}=$ $0.01)$; had a $1.35(P=0.04)$ times higher risk of death; and were $1.24(P=0.09)$ times as likely to fail treatment. However, after adjusting for prognostic factors such as dose, intensity of chemotherapy, number of positive lymph nodes, estrogen receptor status, and age, these researchers found that race was not an independent prognostic factor for overall survival or disease free survival. Roach et al. concluded that they could not "mechanistically" explain the existence of significant biologic breast cancer cause and effect factors related to skin color and that “...efforts directed at early detection of breast cancer in African American women are likely to have greater impact on improving their 
survival than efforts directed at identifying a biologic basis for their lower survival" ( $p$. 111).

Krieger, Van Den Eeden, Zava, and Okamoto (1997) assessed the distributions of breast cancer prognostic biomarkers by race and ethnicity in 48 White, 44 Black, and 43 Asian women diagnosed with breast cancer within a large health maintenance organization in the San Francisco area between 1966 and 1990. The study was based on tumor samples obtained from a random sample of 50 White, 50 Black, and 50 Asian women selected for inclusion in a nested case control study examining the relationship between exposure to organochlorines and risk of breast cancer. Women were included in this study based on self identified race and ethnicity, diagnosis of breast cancer at least six months after receiving a multiphasic examination at the health maintenance organization, and prior to December 31,1990, and the researchers' ability to locate tumor blocks (archival paraffin-embedded tumor biopsy specimens).

A multiphasic self administered questionnaire, supplemented by a physical examination provided data on the women's sociodemographic, reproductive, and anthropometric characteristics. Data were obtained through medical record chart review pertaining to the women's age and parity; menopausal status at the time of diagnosis; tumor characteristics such as stage, grade, laterality, size, and lymph node involvement; and surgical assession number for each cases' tumor block. The researchers reviewed pathology reports to determine which blocks should be analyzed for tumor markers; and immunohistochemistry on thin sections (4 - 5 microns) prepared from paraffin blocks was used to determine biomarkers. The stage of a breast tumor was categorized as local, 
regional, or distant; and tumor size was categorized as $<20$ millimeters or $\geq 20$ millimeters.

Odds ratios and their 95 percent confidence interval and univariate analyses were used to assess sociodemographic, reproductive, anthropometric, and breast cancer tumor characteristic distributions overall and by race and ethnicity. Multivariate logistic regression was used to adjust for place of birth, menopausal status, and age at diagnosis, and to compare tumor characteristic and socioeconomic distributions by race and ethnicity. Approximately 66 percent of the women studied were diagnosed with local disease. Forty percent had tumors that measured $<20$ millimeters, and 44 percent of the women studied had lymph node involvement. There were no notable differences in tumor stage and lymph node involvement across racial and ethnic and socioeconomic groups. When compared to White women, and after adjusting for age at diagnosis, menopausal status, place of birth, and socioeconomic status, Black women were 3.5 times more likely to have breast tumors $\geq 20$ millimeters; and Asian women were 3.7 times more likely to have tumors $\geq 20$ millimeters. After adjusting for age at diagnosis, menopausal status, and place of birth, there was no notable differences in tumor size by socioeconomic status, however tumor size tended to be larger in women who had completed four or more years of college. Approximately 70 percent of the tumors found in all three racial and ethnic groups were estrogen receptor positive; and there were no clear patterns of differences in distribution of breast cancer molecular prognostic markers by socioeconomic class. Therefore, disparities in breast cancer mortality rates between the racial and ethnic groups 
evaluated in this study could not be explained solely by differential distributions of molecular breast cancer prognostic biomarkers.

The studies presented above identify multiple factors influencing the disparity in stage at diagnosis and mortality between African American and Caucasian women. These studies also support the premise that race is not biological, but rather social, cultural, and/or structural. Delayed diagnosis of breast cancer has been shown to occur at a higher rate in the African American women in the studies presented above; however, diagnosis delay has also been shown to be associated with social, cultural, and structural constructs such as SES, educational level, type of health insurance coverage, and obesity. Several of the studies in this section evaluated the relationship between stage at diagnosis of breast cancer, and many of the variables proposed to be examined in this study. Age, race, SES, preventive health practices such as $B S E, C B E$, and mammograms, as well delays related to the diagnosis and treatment of breast cancer, and their relationship to stage at diagnosis outcomes in African American and Caucasian women have been systematically examined . In many instances, the findings were inconclusive. Late stage at diagnosis was repeatedly demonstrated to be associated with African American women. Young African American women, in particular, were found to have more advanced disease and larger tumors. However, many of the African American women in the studies presented above were from low SES; and it is not clear if these differences between the African American and White women in these studies are associated with race, health insurance coverage, or SES. Studies examining access as a multifaceted concept are needed to help provide knowledge and understanding this phenomena. 


\section{$\underline{\text { Socioeconomic Status }}$}

The major factor related to late stage at diagnosis is low socioeconomic status, which has been demonstrated to result in diminished resources and lack of access to health care services (Freeman \& Wasfie, 1989; Griffin, 1994;). The studies presented in this section evaluate the impact of SES on access to breast health care and stage at diagnosis of breast cancer.

Wells and Horm (1992) examined the influence of race and socioeconomic factors on stage at diagnosis of breast cancer in cases of invasive breast cancer in 17,949 White women and 2838 Black women, reported through the U. S. National Cancer Institutes Surveillance Epidemiology and End Results (SEER) program in the San FranciscoOakland, Detroit, and Atlanta metropolitan area from 1978 to 1982 . In situ cases of breast cancer were also available for analysis in this study, 917 among White women and 126 cases among Black women. The researchers chose the median family income strata to ensure that each race group contained enough persons for meaningful analysis; and racespecific indicators of median education and family income were used because Whites and Blacks residing in the same census tract areas were found to have different education and/or income distributions. The Spearman rank correlation coefficient was computed to test the relationship between stage at diagnosis of breast cancer and income and education. The study findings revealed that Black women were diagnosed at a later stage than White women; and that there were 45.7 percent of the Black women diagnosed in localized or in situ stages compared to 52.1 percent of the White women. Eleven percent of the Black women were diagnosed with breast cancer that had metastasized into the distant stage 
compared to seven percent of the White women. The study findings also revealed a less advanced stage with increasing educational level or income among both Black and White women $(\mathrm{P}<.001)$. Black women in the lowest educational and income strata had a poorer stage of disease at presentation than did White women; and Black women in the upper educational and upper income strata had a slightly better stage of disease at diagnosis than White women. Both Black and White women in the upper education and income strata were found to have a higher incidence of breast cancer. This increase in incidence was attributed to the higher incidence rates of in situ and localized breast cancer in this group of women. Incidence of breast cancer in the distant stage was found to decrease with increasing education and income; and the differences between education and income factors influence on stage at diagnosis of breast cancer was found to be more striking in Black women.

Wagner and Schatzkin (1994) used several sources of existing data to determine temporal trends in breast cancer mortality in American women by age, race, and socioeconomic status from 1969 through 1989. County level socioeconomic data relating to the median family income and percentage of persons in a household 25 years of age or older with a high school education, were obtained from the area resource file for the 1970 and 1980 census years. Mortality data was obtained from the compressed mortality file and was also county based. Mortality data on White women was available separately for 1968 through 1989; and on Black women separately for 1979 through 1989. Detailed death records obtained from the mortality files of the National Center for Health Statistics and population estimates provided by the National Cancer Institute were used to obtain 
mortality data for Black women from 1969 through 1972. The study findings revealed that from 1969 through 1972 to 1987 through 1989, mortality climbed 21.4 percent for Black women age 25 to $44 ; 38.3$ percent for Black women age 45 to 65 ; and 54.3 percent for Black women over age 64. For White women, in the same time period, mortality declined 14.4 percent among younger women and 5.1 percent among middle aged women; but mortality increased by 14.7 percent for older White women. The researchers aiso found that breast cancer mortality increasing among both race groups, had risen more rapidly in the lower socioeconomic strata counties. In women 45 to 64 , breast cancer mortality was supported to be declining in women in higher socioeconomic strata counties and rising in lower socioeconomic strata. In women 25 to 44 years of age, breast cancer mortality was demonstrated to be decreasing in both higher and lower socioeconomic strata counties, but at a higher rate of decrease in higher socioeconomic strata counties.

Franzini, Williams, Franklin, Singletary and Theriault (1997) examined the effects of race and SES on survival in 964 White, 205 Hispanic, and 163 Black women treated for breast cancer at the University of Texas M. D. Anderson Cancer Center from 1987 to 1991. Demographic information including race and ethnicity, age at diagnosis, and ability to pay for health care, was collected on the women included in this study at registration. The clinical variables examined in this study included stage of disease at diagnosis, histology, treatment administered in the first four months, and participation in a clinical research protocol. Racial differences in demographic, disease, and treatment characteristics were assessed using odds ratios and their corresponding 95 percent confidence intervals. The Mantel-Cox test was used to assess differences in the survival 
curves between subgroups; and the independent effects of race, age, stage, treatment, SES, and histology on survival was assessed using the multivariate Cox proportional hazards model, after adjusting for the effects of all other factors included in the model. Overall there was no notable difference in the age distribution between Black and White women but Hispanic women were diagnosed at younger ages. Black and Hispanic women were less likely to be in high SES groups, and Black women tended to be poorer economically when compared to Hispanic women. When compared to White women, early stage disease was less prevalent among Hispanic and Black women. Black women tended to have more distant metastasis when compared with Hispanic women and a higher percentage of Hispanic women had disease that involved lymph nodes. The differences in treatment by either race or SES were negligible when adjustments were made by stage of breast cancer at diagnosis. Histological type did not differ by race. There were significant $(P=0.0005)$ differences in survival by race. The differences continued to be significant across race $(P=0.02)$ when stratified by stage, but differences in survival by race were eliminated when both stage at diagnosis and SES were controlled for $(P=15)$. Women in lower SES had significantly poorer survival $(\mathrm{P}<0.0001)$. Adjusting for stage at diagnosis $(P=0.003)$ and for stage and race $(P=0.008)$ did not eliminate these significant survival differences. Race and histology were not significant independent predictors of survival; however, age, stage at diagnosis, protocol participation, treatment choices, and SES were all found to be significant independent predictors of survival $(P<0.05)$. When the relationship between race and SES was investigated, the excess risk for Black women was found to be 98 percent when compared to White women. The excess risk decreased to 
non-significance at 34 percent in Black women when all prognostic variables were controlled. Prognostic variables explained 66 percent of the unadjusted excess risk in Black women in this study; stage at diagnosis explained 38 percent, and SES explained 45 percent.

Heck, Wagner, Schatzkin, Deveas, \& Breen (1997) assessed the relationship between socioeconomic status and breast cancer mortality by examining educational levels on death certificates of women dying between 1989 and 1993. Death certificates of women 25 years or older, whose death certificate listed breast cancer as the underlying cause of death were included in the study. Ethnic groups were categorized as Hispanic, non Hispanic White, non Hispanic Black, and non Hispanic Asian. Educational levels were categorized as 0 through 8 years, 9 through 11 years, 12 years, 13 through 15 years, and 16 or more years of education. Data were collected by review of death certificates and analyzed using the SAS program. The researchers found that women with fewer than 12 years of education had lower mortality rates than women with at least twelve years; and the highest rate of breast cancer mortality was found among women with 12 years of education and among those with 16 years or more of education. Asian women in this study had the lowest age adjusted mortality rates, while Black non Hispanic women had the highest rates, followed by White non Hispanic women. Within each non White population of this study, the mortality rate for breast cancer increased with greater education through 12 years. The mortality rate for non Hispanic Black women with 16 or more years of education was similar to that of women with 12 years, but lower than that of women with 13 to 15 years. The non Hispanic White women included in this study did 
not demonstrate a strong positive gradient of mortality by educational level. The findings of this study are interesting and vary from findings relating to educational level presented earlier in this section. In addition, the assumption was made that educational level was an appropriate measure of SES and that the educational level of the decedent as listed on the death certificate was accurate. However, the results of this study support the inclusion of SES as a variable in this study.

The studies presented above support a significant relationship between low SES and delayed diagnosis of breast cancer. Other factors such as age, educational level, stage at diagnosis of breast cancer, tumor characteristics, and treatment modalities, were also found to be significant predictors of breast cancer outcomes. In Black women high educational levels were associated with both early stage at diagnosis and high mortality. Delayed diagnosis of breast cancer was demonstrated to be more prevalent in African American women when compared to White women; but since low SES seemed to be more prevalent in the African American women than in the White women included in these studies, it is not clear if the poorer outcomes in African American women are associated with race, SES, or a combination of the two. Nevertheless, there is strong support for the inclusion of SES in this study as a variable of potential access

\section{Means of Discovery of Breast Cancer}

The American Cancer Society and the National Cancer Institute recommend three strategies for early detection of breast cancer: mammography, clinical breast exam (CBE), and breast self exam (BSE). Mammography has been promoted as the optimal prevention strategy for breast cancer, and its effectiveness as a screening procedure for detecting 
breast cancer at an early stage has been well documented in the literature (Champion \& Miller, 1996; Crooks \& Neutens, 1993; Frazier, Jiles, \& Mayberry, 1996; Long, 1993; Miller \& Champion, 1996; Thomas, Fox, Leake, \& Roetzheim, 1996 ). Mammography results have been estimated to be $75-90$ percent effective in detection of breast abnormalities, and mammography used in conjunction with CBE has been proven to be very effective. Although not recommended as the only breast cancer screening activity, BSE has also been proven effective in discovery of breast abnormalities.

BSE is a useful method because (a) most tumors are found by women themselves either accidentally or during self examination; (b) retrospective data show that patients diagnosed at earlier stages of breast cancer were more likely to have practiced regular BSE than patients diagnosed at later stages; (c) the cost benefit ratio of mammography may not be advantageous for women $<40$ years of age who have a lower incidence of breast cancer than older women; (d) mammography screening may not be readily available to all women, especially those in rural and poor inner city locations; and [(e)] screening mammography is recommended every 1-2 years for women $\geq 50$, thus creating a need for BSE practice between mammographies (Han, Baumann, \& Cimprich, 1996, p. 197).

Therefore, mammography conducted in conjunction with CBE and BSE is the recommended approach for effective breast cancer screening and early diagnosis of breast cancer.

The relationship between breast cancer survival and early stage at diagnosis is well documented in the literature (Chu et al., 1996; Harris, Lippman, Veronesi, Willett, 1992; 
Kirkwood, Lotze, \& Yasko, 1996; McPherson, Swenson, Jolitz, \& Murray, 1997:

Pederson \& Trigg, 1995; Phillips, 1991). Early detection and treatment of breast cancer is extremely important, and breast cancer diagnosis initiated by breast cancer screening activities (BSE, CBE, or mammography), is more likely to be diagnosed in an earlier stage than breast cancer discovered incidentally or secondary to some other medical condition. Numerous studies have looked concurrently and individually at African American and Caucasian women's participation in breast cancer screening, and have identified access, cultural, social, personal, and economic barriers to participation in breast screening activities and breast health care programs (Adderley-Kelly \& Green, 1997; Bakker, Lightfoot, Steggles, \& Jackson, 1998; Bowen, Hickman, Powers, 1997; Brown \& Williams, 1994; Burnett, Steakley, \& Teff, 1995; Champion, 1992; Champion \& Miller, 1996; Champion \& Menon, 1997; Champion \& Scott, 1997; Crane, Kaplan, Bastani, \& Scrimshaw, 1996; Crooks \& Neutens, 1993; Duke, Gordorn-Sosby, Reynolds, \& Gram, 1994; Erwin, Spatz, Turturro, 1992; Frazier, Jiles, \& Mayberry, 1996; Glenn \& Moore, 1990; Han, Bauman, Cimprich, 1996; Hoffman-Goetz \& Mills, 1997; Kurtz, Given, Given, \& Kurtz, 1993; Lerman, Rimer, Trock, Balshem, \& Engstrom, 1990; Lierman, Young, Kasprzyk, \& Benoliel, 1990; Lierman, Young, Powell-Cope, Georgiadou, \& Benoliel, 1994; Mandelblatt, et al., 1993; Miller \& Champion, 1996, 1997; Miller \& Hailey, 1994; Morgan \& Levin, 1995; Nemcek, 1990; Olsen \& Frank-Stromborg, 1994; Olson \& Morse, 1996; Patterson, Helvie, Joynt, Roubidoux, \& Strawderman, 1998; Persson, Svensson, \& Ek, 1997; Phillips \& Wilbur, 1995; Sensiba \& Stewart, 1995; Tessaro, Eng, \& Smith, 1994; Thomas, Fox, Leake, \& Roetzheim, 1996; Thompson, 
Montano, Mahloch, Mullen, \& Taylor, 1997; Valdini \& Cargill, 1997; Williams, Abbott, \& Taylor, 1997) . However, these studies have not examined the impact of these barriers on stage at diagnosis or breast cancer mortality. The studies listed above focused on uncovering reasons why women did or did not participate in breast health programs, and on identifying intervention strategies to increase involvement in breast cancer screening activities. Few studies have looked primarily at the relationship between participation in breast cancer screening activities and stage at diagnosis of breast cancer; and even fewer studies have examined the impact of race or economic access on this relationship. Several studies have examined differences in participation in breast cancer screening by race, secondary to exploring the relationship between race, SES, economic access, and stage at diagnosis of breast cancer (Franzini, Williams, Franklin, Singletary, \& Theriault, 1997; Eley et al., 1994; Hunter et al., 1993; Newman \& Alfonso, 1997; Wells \& Horm, 1992). The results of these studies were presented earlier in the literature review section. Therefore, the following section includes studies which concurrently examine breast cancer screening activities in African American and Caucasian women in relation to breast cancer diagnosis and survival outcomes. Studies looking primarily at BSE or CBE and their relationships to stage at diagnosis in African American and Caucasian women could not be located. Examination of $\mathrm{CBE}$ practices was consistently included in studies examining use of mammography. Therefore, the following section is comprised of studies which examine all three screening activities, and studies focusing primarily on utilization of CBE and mammography in samples composed of African American and Caucasian women. 
Vernon et al. (1992), used data from the 1987 American Cancer Society Texas Breast Screening project to examine attitudes and behaviors related to breast cancer screening in a multiethnic sample of women. The study included a population of 31,000 White, 1800 Hispanic, and 1100 Black women age 35 and older. There were more Black (30 percent) and Hispanic ( 28 percent) women in the younger 35 to 39 age group than White women (17 percent); and Black (16 percent)and Hispanic (16.4 percent) women had proportionately more women in the lowest $(<13,000$ annual household income) income category, when compared to White women (8.6 percent). A higher proportion of Hispanic women had less than a high school education (19.2 percent), when compared to Black ( 9 percent) and White ( 7 percent) women. Vernon et al. used the chi-square test to compare the frequency of breast cancer screening behaviors across these groups of women and found that White women (33 percent) were more likely than Black (27 percent) and Hispanic (24 percent) women to have had a mammogram prior to participating in the screening project. Income level was associated with prior mammography only in the White women, and educational level was not associated with prior mammography in any of the ethnic groups. In all three groups, women who had prior CBE were more likely than women who had not had prior CBE to report prior mammography. White and Hispanic women who practiced BSE were more likely than women who did not practice BSE to have had prior mammography. This pattern was also observed in the Black women in this study but was not statistically significant $(P=0.078)$. In all groups, women with a history of breast cancer in their family were more likely to have had a mammogram prior to the screening project than those without a history. The two most important 
reasons for not having had a prior mammogram given by the women in this study were cost and lack of physician referral. Lack of physician referral ranked first for Black and White women; and cost ranked first for Hispanic women. Cost, as a reason for not having had a prior mammogram, was inversely associated with educational level in all three ethnic groups.

In a descriptive study, Douglass, Bartolucci, Waterbor, and Sirles (1995), administered the Breast Cancer Control and Health Beliefs Questionnaire to a group of 117 African American and 157 White women educators employed by a public school system, to identify differences in health beliefs and practices regarding mammography, $\mathrm{CBE}$, and BSE. Women included in the study were recruited from a list of randomly selected schools, within a selected school system, within a selected state, based on current employment as a professional educator, age 35 years or older, and no personal history of breast cancer. The mean age of the sample was 47.62 for the African American women and 44.71 for the White women. The SES score was calculated using Green's two-factor procedure, and the difference in scores for the two groups of women was not statistically significant. The chi-square test was used to test for significant differences between the two groups on frequency of use of mammography, BSE, and CBE. The difference for the frequency of use of mammogram and $\mathrm{CBE}$ was not statistically significant $(\mathrm{P}=0.735 ; \mathrm{P}=$ 0.083 respectively). The difference for $\mathrm{BSE}$ frequency approached significance $(\mathrm{P}=$ 0.058), with a larger proportion of the African American (41 percent compared to 31 percent) women reporting performance of BSE during the previous 12 months. Analysis of variance was used in the calculation of differences in health belief scores between the 
two groups. When compared with African American women, White women had a significantly higher $(\mathrm{P}=0.002)$ mean score for perceived barriers to mammography and $\mathrm{CBE}(\mathrm{P}=.05)$. The researchers looked at race as a predictor of the use of breast cancer screening modalities using logistic regression, and found no significant differences $(\mathrm{P}=$ $0.50-0.99)$ in the ability of the health belief model to predict use of mammography, CBE, or BSE, according to race.

Michels, Carter, Taplin, and Kugler (1995) examined barriers to mammography use in women eligible for military health care at Madigan Army Medical Center in Washington state. Data were collected using a questionnaire developed by the researchers. A random sample of zip codes in the Madigan Medical Center service area was obtained through the Defense Eligibility Enrollment Reporting System (DEERS) and 500 questionnaires were mailed to women 40 years or older, who had been randomly selected based on two of the digits in their social security numbers. A total of 440 women responded to the survey, and 309 of those responding were eligible for inclusion in the study. Chi-square, analysis of variance, and Pearson's correlation were used to conduct statistical analysis. The age of the respondents ranged from 41 to $89 ; 72.4$ percent of the women were white, 10.7 percent African American, 15.9 percent Asian American, 3.7 percent Hispanic, and 1 percent were of other ethnic origin. The proportion of women with less than a high school education was 22.6 percent, while the proportion of women with greater than a high school education was 13 percent. The proportion of women with annual income levels less than $\$ 10,000$ was 19.6 percent, and the proportion with income levels greater than $\$ 50,000$ annually was 2.1 percent. A total of 21.5 percent of the 
women in the study had never had a mammogram; 39.2 percent had a mammogram within the past year, 56.4 percent within the past two years, and more than five years had passed since 10.8 percent of the women in this study had had mammograms. Women with higher income levels, with a higher risk factor for breast cancer, and with a higher perceived risk of breast cancer were more likely to have participated regularly in mammography. Women with a higher rate of mammography participation were more likely to state that their provider or the media recommended mammography. The researchers did not detect any difference between women with higher participation and all other women in the study based on age, race, education, insurance coverage for mammography, or military versus civilian source of care. Overall, 12.8 percent of the women included in this study participated in regular mammography, compared to 31 percent of the women in the general population.

Benedict, Williams, and Hoomani (1996) conducted a retrospective study of women diagnosed with breast cancer to determine the means of discovery according to adherence to American Cancer Society (ACS) guidelines for BSE, CBE, and mammography; and according to age, race, family history and educational level. A questionnaire with demographic questions, questions about the women's breast cancer detection practices, and method of discovery of breast tumor was developed and mailed to women from the mailing list of a breast cancer support group in Charleston, South Carolina. Of the 190 questionnaires mailed, 188 were returned and 51 were selected for inclusion in the study based on the time of diagnosis fitting within a 1990 to 1992 time frame. Nine of the women included in the study were Black and 41 were White. The 
mean age at the time of diagnosis for the Black women was 49.75 years as compared to 50.08 years for the White women. The mode of highest educational level for the Black women was "some college" (44\%) as compared to "junior high school" (26.8\%) for the White women; and 22.2 percent of the Black women had a family history of breast cancer as compared to 19.5 percent of the White women. Method of discovery according to stage at diagnosis was extracted from the questionnaires of 20 women. Of the stage 1 cancers diagnosed in the women in this study $(n=13), 61.5(n=8)$ percent were discovered by mammogram, as compared to $23.1(n=3)$ percent by BSE, $7.7(n=1)$ percent by accident, and $7.7(n=1)$ percent by another person. One-hundred percent of the stage 3 $(n=2)$ cancers were discovered by BSE, and stage $4(n=2)$ discoveries were equally divided (50\%) between BSE and CBE. Within the stage $2(n=12)$ grouping, $58.3(n=7)$ percent of the discoveries occurred by BSE, $16.7(\mathrm{n}=2)$ percent by accident, and $16.7(\mathrm{n}=2)$ percent by mammogram. Of the Black women included in the study, 11.1 percent of the cancers were discovered by BSE, 33.3 percent by accident, and 55.6 percent by mammogram. Of the White women in the study, 34.1 percent of the cancers were discovered by BSE, 7.35 percent by accident, 31.7 by mammogram, 7.3 percent by $C B E$, and 7.3 percent by another person. Twenty-two of the Black women in the study had mammograms every 1 to 2 years before diagnosis, as compared to 56 percent of the White women, and 55.6 percent of the Black women performed BSE in the 12 months prior to diagnosis as compared to 51.2 percent of the White women. Because of the small sample size of Black women, and the unequal group size (Black and White women), inferential statistics were not conducted. 
O'Malley, Earp, and Harris (1997) used data from the New Hanover Breast Cancer Screening program, a National Cancer Institute Consortium project conducted between 1987 and 1991 in two eastern North Carolina counties, to examine racial differences in mammography use in Black and White women. The researchers obtained data from a 1988 random digit dialing telephone survey of 1214 women age 30 to 74 years. A total of 948 Black and White women age 50 to 74 years were included in this study. Chi-square tests, $t$ tests, Wilcoxon rank sums, Kruskal-Wallis chi-square approximations, and logistic regression analyses were used to compare attitudes and characteristics by race and by mammography use. Approximately 90 percent of the women included in this study were aware of mammography; 46 percent reported a prior mammogram; and 32 percent reported having a mammogram in the past year. Black women reported ever having had a mammogram ( 27 percent) approximately half as often as White women ( 52 percent); and White women reported having had a physicians' recommendation for mammography twice ( 52 percent) as often as the Black women (25 percent) in the study. When factors related to race and mammography use were controlled for, the initial racial differences in mammography use were eliminated. Physician recommendation alone accounted for 60 to 75 percent of the mammography use differences by race.

In a cross sectional descriptive study, Dibble, Vanoni, and Miaskowski (1997) examined women's attitudes toward breast cancer screening procedures from ten sites in a major metropolitan area in California. The study was conducted with a convenience sample of 233 White, 127 African American, 118 Pacific Islanders, 61 Hispanic, and 58 
Asian American women age 18 years or older, recruited from public health centers, mobile mammography vans, and hospital based radiology departments. Data were collected using a demographic questionnaire and the Attitude Toward Breast Cancer Screening Procedures Scale. Differences in women's attitudes by ethnicity were calculated using descriptive statistics, analysis of covariance, and the Tukey post hoc analysis procedure. Mean scores of the demographic characteristics of the sample were also calculated and included in the data analysis. The mean age of the participants was 53 years; 48 percent were married or partnered; 81 percent had children; 62 percent were currently employed; and 44 percent had an annual family income of less than $\$ 20,000$. The average years of education was 13.9. Seventy-nine percent of the women had had a previous mammogram; and 4 percent had been previously diagnosed with breast cancer, while 23 percent had experienced a lump in their breast. After controlling for age, education, number of pregnancies, marital status, income, employment status, previous mammogram, lump and family history, significant differences by ethnicity $(F=6.65, P<.0001)$, in the scores on the Attitude Toward Breast Cancer Screening Procedures Scale were detected. The African American women in this study had the most positive attitude towards breast cancer screening procedures.

Roberson (1997), compared breast cancer screening outcomes among 282 African American and 1079 White women participating in a multistrategy cancer control intervention in western New York. Data were collected from women who participated in the intervention between mid 1994 and mid 1996 using a screening intake form. Data collected included demographic information, as well as information regarding health 
practices, risk factors, screening status, and clinical findings. The mean age for African American women included in the study was 50 years as compared to 56 years for White women. Twelve or more years of high school had been completed by 79 percent of the African American women, as compared to 82 percent of the White women. Across both groups, annual family incomes of less than $\$ 15,000$ were reported in 99 percent of the women studied. A significant difference in the health insurance status of African American and White women was noted $(\mathrm{P}<.001)$. Twenty-four percent of the African American women reported Medicare and Medicaid as their primary source of health care insurance, while 75 percent reported having no health insurance coverage. Twenty-five percent of the White women reported having Medicare and Medicaid as their source of health care insurance, and 53 percent reported having no insurance at all. Sixty-one percent of the White women in the study reported that they had a previous mammogram as compared to 53 percent of the African American women $(\mathrm{P}<.002)$. Twenty four percent of the White women reported that they practiced BSE monthly as compared to 33 percent of the African American women included in this study $(P<.009)$. Twelve percent of the African American women presented with breast abnormalities that were probably benign and required short term follow-up, as compared to 11 percent of the White women; and 6 percent of the African American women were found to have breast abnormalities by mammogram, as compared to 5 percent of the White women in this study. This study was limited in that it studied a sample of relatively low-income women. However, the study found that women in this low income group, who participated in cancer control interventions, were similar in educational and income levels. This study supported 
findings of other studies presented above, in that the African American women, in this study, were more likely to participate in BSE than were the White women. However, the White women in this study were slightly more likely to have had a previous mammogram than the African American women.

In the studies presented above, race was not a significant predictor of participation in breast cancer screening programs. In two of the studies, African American women were found to be more likely to participate in breast cancer screening than White women; and African American women were found more likely to practice self exam than White women. In another study, even though the percentage of mammogram discovered breast cancer was higher for the African American women than for the White women, African American women were found to have had a lower percentage of mammography screening participation prior to breast cancer diagnosis, when compared to White women. Barriers to accessing breast cancer screening were attributed to the individual as well as the health care delivery system. In the study conducted on mammography use by women eligible for care within the MHS, mammography rates were found to be lower than those in the United States overall. Income level and cost of mammography were primary factors associated with prior mammography use by the women in the studies presented above. Physician referral was also demonstrated to be a major factor associated with mammography use; and physician referral is associated with economic access to care. Even though most of the studies presented above did not present results relating to the association between the particular means of discovery of breast cancer and stage at 
diagnosis of breast cancer, there is support for inclusion of this variable (the means of discovery of breast cancer) in this study, as a measure of potential access.

Realized Access and Stage at Diagnosis of Breast Cancer

Evaluation of the utilization of health services by the consumer is a key factor in examining health care access; and the willingness of the consumer to seek care is crucial, if potential access is to become realized access (Aday \& Andersen, 1974; Aday, Andersen, \& Fleming, 1980; Aday, Fleming, \& Andersen, 1984). The disposition of the consumer to utilize services is influenced by economic access concerns and social and cultural definitions of health and illness; as well as, attitudes towards and knowledge of health care (Adams, 1995; Bayne-Smith, 1996; Donnelly, 1995; Frank-Stromborg \& Olsen, 1993; Jennings, 1996; Kagawa-Singer, 1995. 1997; Olsen \& Frank-Stromborg, 1993; Palos, 1994). As a result, economic access and realized access are not always synonymous, and timeliness in the utilization of services, by the consumer, can become an important factor in determining health care outcomes.

Women vary in their response to the discovery of breast cancer symptoms. Some women seek provider attention and treatment immediately upon discovery, and other women delay diagnosis and treatment for greater than two years (Facione, 1993; Facione, Dodd, Holzemer, \& Meleis, 1997; Katz, Hislop, Thomas, \& Larson, 1993; Lauver, 1992a, 1992b; Lauver \& Angerame, 1993; Lauver \& Ho, 1993; Lierman, 1988; Vernon, Tilley, Neale, \& Steinfeldt, 1985). The relationship between delayed diagnosis and treatment of breast cancer, and increased mortality, has been well established (Bedell, Wood, Lezotte, Sedlacek, \& Orleans, 1995; Hunter et al., 1993; Long, 1993; Mandelblatt, Andrews, Kao, 
Wallace \& Kerner, 1995; Simon \& Severson, 1996); and has been presented in earlier sections of this literature review. Therefore, the following section focuses on studies examining factors associated with diagnosis and treatment delay of breast cancer, in samples including both African American and Caucasian women.

\section{Diagnosis Delays}

Coates et al. (1992) explored differences in the length of time between symptom recognition and initial medical consultation between 410 Black and 325 White women, newly diagnosed with breast cancer in 1985 or 1986, from the Atlanta, New Orleans, and San Francisco/Oakland areas. The researchers conducted in person interviews to obtain information specific to race and recognition of breast cancer symptoms; and collected retrospective data on symptoms, dates of recognition, and initial medical consultation. Tests used to conduct the statistical analyses included the Mantel-Cox test, Cox proportional hazards regression analysis, and logistic regression analysis. Women who had their breast cancer diagnosed prior to symptom recognition were more likely to have been White and older; to have smaller, earlier stage, lower grade tumors; and to have lived in the San Francisco/Oakland area. White women were more likely than Black women to have obtained medical consultation on each day after the first three days following symptom recognition; and 32 percent of the Black women, compared to 40.3 percent of the White women had obtained medical consultation by the seventh day following symptom recognition. The difference in the median symptom duration was of borderline significance $(\mathrm{P}=.06)$ at 16 days for Black women compared to 14 days for White women. Even though Black women were two times more likely than White women (6.6 percent 
compared to 3.1 percent) to have stage IV breast cancer when diagnosed, and one and onhalf times more likely to have stage III breast cancer when diagnosed than White women (21.2 percent compared to 13.5 percent), the small racial difference in time between recognition of symptoms and initial medical consultation could not explain the racial differences in stage or tumor size at diagnosis.

Lauver, Coyle, and Panchmatia (1995) assessed women's reasons for and barriers to seeking care for breast cancer symptoms in a multiethnic sample of 138 women recruited from a breast clinic in an urban teaching hospital providing care to the medically indigent. The women included in the study varied in age from 19 to 76 years. Sixty-three of the participants were Caucasian, 71 were African American, and 4 were Asian and Latina. Thirty-two percent of the women had not finished high school as compared to 30.4 percent with a high school education. Of the women included in the study 22.5 percent had completed some college and 15.2 percent had finished college. The median annual family income of the women was $\$ 12,000$, with a range of $\$ 0$ to $\$ 300,000$. In regard to health care insurance, 58.7 percent of the women were covered by public assistance; 26.8 percent by private insurance; 3.5 percent by private and public monies; 3.6 percent by Medicare; and 7.4 percent did not report a source of health insurance coverage. The researchers determined delay in seeking care by calculating the number of days between the woman finding a breast change and the woman's contact with the health care delivery system, concerning the change, by telephone or in person. Twenty-three percent of the women were discovered to have delayed three months or longer. Thirtyeight percent of the women sought care within one week of symptom discovery; 49 
percent within two weeks; 65 percent within six weeks; and 77 percent within three months. Fifty percent of the women reported no barriers to seeking care for breast changes, and sought care sooner (mean $=53$ days, median $=11$ days) than those women citing particular barriers to care (mean $=143$ days, median $=32.5$ days). The barrier most commonly cited as interfering with care seeking was conflicting commitments (15.8 percent of responses); followed by limited finances/insurance (11.8 percent); fear or avoidance ( 9.9 percent); transportation problems ( 6.6 percent); lack of knowledge of how to access the system ( 3.3 percent); and other reasons ( 2.6 percent).

Caplan, Helzlsouer, Shapiro, Wesley, and Edwards (1996), examined reasons for system delay in breast cancer diagnosis, from the initial medical consultation to establishment of a diagnosis, in women from the National Cancer Institute's Black/White Cancer Survival Study. There were 1,222 women eligible for inclusion in the Black/White Study. Data collection methods for the study included medical record review, centralized pathology review, and personal interview. The personal interview collected data on previous health practices, symptom recognition, care seeking behaviors, dietary patterns, personal health practices, attitudes toward the health care system, SES, and reasons for delay. Women were included in this study based on a system delay of four or more weeks between the initial medical consultation for a breast cancer symptom, and diagnosis of breast cancer. These inclusion criteria yielded a sample of 367 women. The Chi-square test was used to compare the Black and White women regarding basic demographics, symptom status, stage at diagnosis, and delay. Fifty-five percent of the study sample was Black, and most of the study sample used a private physician as their primary source of 
care. The White women were significantly more likely than the Black women to be asymptomatic at the time of initial presentation; and Black women were significantly more likely than White women to have advanced disease. The median system delay for Black women was 11 weeks as compared to 13 weeks for White women. Most women in both groups (45 percent) attributed delay to the health care system, due to physician inaction or difficulty scheduling appointments. When compared to White women, more Black women attributed system delays to scheduling delays, rather than to physician inaction. Women attributing delay in diagnosis to themselves ( 25 percent) tended to have more advanced disease. The reason most often given for delay by both groups of women was that the problem was not important or urgent (37.6 percent); followed by the event of a birthday, holiday, or out of town commitment (17.8 percent); competing health problems (14 percent); being too busy (12.7 percent); and fear (12.7 percent). Seventeen percent of the women in the study attributed delay to both themselves and the health care delivery system.

Even though the studies presented above primarily examined the length of time between discovery and initial contact with the health care delivery system, there is support for the inclusion of the length of time between discovery of a breast abnormality and the diagnosis of breast cancer as a measure of utilization of health care services, in this study. Mean score diagnosis delays were comparable for African American and White women with no significant differences being reported above. Reasons for delay were attributed to characteristics of both the individual and health care delivery system. Even though the perception of barriers was found to delay care seeking in one of the studies presented 
above, the barrier most frequently cited in two of the studies in this section related to characteristics of the individual, rather than characteristic of the health care delivery system. Advanced disease was found to be higher in African American women than in White women in one of the studies; however, there was no significant difference in mean diagnosis delay scores between these two groups.

\section{Treatment Delays}

Bedell, Wood, Lezotte, Sedlacek and Orleans (1995) examined delay in diagnosis and treatment of breast cancer in 225 women diagnosed with breast cancer between January 1, 1992 and December 31, 1993. The Colorado Central Cancer registry was used to identify women and data were collected from the medical records at three sites: a public hospital, a university hospital cancer center, and a private practice. Data were abstracted from the records of 52 women from the public hospital, 83 women from the university hospital, and 90 women from private practice, and included information on age at diagnosis, race, insurance status, stage at diagnosis of breast cancer, the initial breast cancer symptom, the date of the first medical consultation, the date of the biopsy for definitive diagnosis, and the date of the initiation of treatment. The analysis of the data focused on the length of time in days between the first medical consultation and biopsy for definitive breast cancer diagnosis, and the length of time in days between definitive diagnosis and the initiation of treatment. Sources for delay were divided into three categories. System delay referred to delay resulting from routine practices of the specific health care delivery system, and included such sub-categories as scheduling delay or time spent waiting for appointments; cancellation or postponement of appointments; delay in 
reporting examination or test results to the provider; and delay attributed to lost or unavailable records. Provider delay referred to delay relating to physician or other health care provider behaviors, such as a work-up exceeding 14 days; a justifiable delay occurring when evaluation had not revealed cancer but the case was being closely followed; and misdiagnosis of cancer symptoms. Patient delay referred to delay relating to patient behaviors. Patient delay included sub-categories such as a prolonged period of decision making; refusal of further medical evaluation or treatment; concerns related to finances; scheduling rearranged for the patient's convenience; and the patient's seeking a second opinion. The Wilcoxon rank sum test and chi-square were used to conduct the statistical analysis. The majority of the women from the public hospital were Hispanic. African American women were seen more often at the public hospital than at the two other sites combined; and nearly half of the women at the public hospital and the university hospital were uninsured. Five percent of the women from private practice were uninsured; and all three sites had similar proportions of women with Medicaid or Medicare as their source of health care insurance. The ages of the women in this study ranged from 27 to 90 years with a mean age of 53.3 . The diagnostic interval median was significantly different among the three sites $(P=0.003)$. The diagnostic interval median at the public hospital was 25 days, as compared to 14 days in private practice, and 24 days at the university hospital. The treatment interval median was also significantly different among the groups $(P=0.029)$, with a median of 15 days in the public hospital, 10 days in private practice, and 13 days at the university hospital. During the diagnostic interval, differences in delay among the three groups was significant only for the system category $(\mathrm{P}=0.038)$. 
There were longer intervals of delay for the public hospital than for private practice $(\mathbf{P}=$ $0.036)$ or the university hospital $(P=0.018)$. However, during the treatment interval, significant differences in delay among the three sights were found by system $(P=0.04)$ and by patient $(\mathrm{P}=0.019)$. There were no significant differences across the three sites in the treatment interval, by provider. Patient caused delay, during the treatment interval, occurred more often in private practice than at any other site. A major finding of this study was the significant difference observed in diagnosis delay between the public hospital and private setting. Over 50 percent of the diagnostic delay occurring in the public hospital was attributed to system factors. Even though this finding is enlightening, the researchers did not include stratification of the sample by race in their report of findings, nor did they evaluate the relationship between the diagnosis delay found in the public hospital, and the stage of diagnosis of those women receiving care in that particular setting. Nevertheless, the study results support variance in breast cancer diagnosis and treatment intervals, by type of health care delivery setting. Even though it is not clear if the difference in patient delay occurring in the public hospital is influenced by system factors, source of health insurance coverage, or individual characteristics of the patient, this study supports the inclusion of both diagnostic and treatment delays as measures of realized access.

\section{Conclusion}

The review of literature presented above supports the research design for this study, and the inclusion of each of the variables in this study's conceptual framework. In addition, the review supports the relationship between economic access to breast health 
care and stage at diagnosis of breast cancer. Variation in breast cancer outcomes was found to be associated with the type of health care insurance coverage; but even when health care insurance was comparable within a study population, stage at diagnosis varied by age, SES, the means of discovery of breast cancer, and diagnosis and treatment delays. Multiple factors are associated with stage at diagnosis of breast cancer. These factors are components of both potential and realized access. Age, race, and SES were found to be related to stage at diagnosis and participation in breast cancer screening; and participation in breast cancer screening was found to be related to utilization of health care services. Even though no studies could be located which specifically addressed the relationship between participation in breast cancer screening and stage of breast cancer at diagnosis, the literature presented above supports that women who participate in breast cancer screening activities are more likely to be diagnosed with early stage breast cancer than those who do not. Diagnosis and treatment delays were found to be associated with characteristics of the health care delivery system, as well as characteristics of the individual. Therefore, the studies presented above support the relationship between potential and realized access.

Many of the studies presented above used existing data to conduct retrospective analyses. Several of the studies conducted retrospective analysis using the same data base. Data from the National Cancer Institute's Black/White Study was analyzed by several different groups of investigators. However, each group addressed a set of different research questions; and the variety of inclusion criteria used created a unique sample for each study. In addition, the sample sizes and statistical tests were appropriate for the 
research design and the questions being addressed. Therefore the results from the studies using the Black/White Study database are felt to be valuable in supporting the existence of a relationship between the variables included in this study's conceptual model.

Other studies collected data from a single health care institution, or from a specific socioeconomic strata or educational level. Even though these data are limited in their generalizability, the appropriateness of the sample size and statistical tests, to the research design, make the results of these studies valuable to the construction of the research design of this study.

Although several of the studies included in this review examined the majority of the variables included in the conceptual model for this study, in aggregate, no one study examined all of the variables proposed for examination in this study, within an equal economic access health care setting. Economic access barriers such as health care insurance and availability of health care resources were demonstrated to be related to delayed diagnosis of breast cancer. However, demographic characteristics, as well as personal barriers to participation in breast cancer diagnosis and treatment were also found to be related to stage at diagnosis. Even though the review of literature offers support for relationships between all of the variables in this study's conceptual model, the impact of equal economic access on variables of potential and realized access, and stage at diagnosis of breast cancer, is unclear.

In summary, the review of literature presented above indicates that research is needed to assess the relationship between access (economic, potential, and realized) and stage at diagnosis of breast cancer in women being treated for breast cancer inside the MHS. Descriptive comparative studies conducted in settings in which health care 
insurance coverage is equal, and distribution of health care resources is adequate, are needed to generate a better understanding of the multiple factors influencing disparity in stage at diagnosis between African American and Caucasian women. This knowledge can lead to hypotheses development and testing for the purpose of identifying appropriate interventions to increase access to breast cancer screening for all American women. 


\section{CHAPTER III}

\section{METHODOLOGY}

The research design, the setting, the population, and the sample are described in this chapter. The methods and procedures for data collection, as well as the data analysis techniques are also described.

\section{Design}

The purpose of this study was to describe the relationship between access and stage at diagnosis in African American and Caucasian women being diagnosed with breast cancer within the DoD MHS. There was no experimental manipulation of variables or random assignment of subjects, and the data were collected through secondary analysis. The research design for this study was non-experimental, descriptive-comparative (Burns \& Grove, 1993).

\section{Setting}

The setting for this study was the Naval Medical Center, San Diego California (NMCSD), a health care facility within the DoD MHS. Data were collected in the NMCSD Tumor Board Registry, and the source of the data were tumor registry records compiled and maintained at NMCSD. The following section provides an overview of: (a) the structure of the NMCSD, (b) the beneficiary population served by NMCSD, and (c) the structure and functioning of the NMCSD Tumor Board Registry.

Overview of NMCSD. The medical center is a 535 bed acute care military 
treatment facility with an occupancy rate of 66.3 percent and an average daily census of 254 (Region Nine TRICARE, 1998). Constructed in 1987, NMCSD has the ability to expand to 696 beds in contingency situations. In 1995, NMCSD had a total of 1,014, 942 outpatient visits and filled $1,280,002$ outpatient prescriptions. The medical center is staffed by civilian and military personnel. In 1995, staffing included 707 clinician full time equivalents (FTEs), 521 registered nurse FTEs, 226 allied health professional FTEs, 2103 para-professional FTEs, and 1095 administrative FTEs. The medical center is accredited by the Joint Commission for Accreditation of Hospital Organizations (JCAHO).

Beneficiary Demographics. There are a total of 257,658 beneficiaries eligible to receive health care at NMCSD: 118,065 females and 139,593 males (Defense Eligibility Enrollment Report System [DEERS], 1997). Thirty-one percent of the eligible beneficiaries are family members of active duty, 24 percent are active duty, 22 percent are family members of retired, 17 percent are retired, 4 percent are survivors of retired, 1 percent are guard reserve, and 1 percent are family members of guard reserve. Thirty-nine percent of the females are ages 18 to 44,35 percent are ages 45 and older, and 26 percent are ages zero to 17 . Forty-eight percent of the males are ages 18 to 44,29 percent are ages 45 and older, and 23 percent are ages zero to 17. According to the DEERS (1997) database, 26 percent of those beneficiaries eligible for care at NMCSD are of unknown race. Of those beneficiaries with known race, 43 percent are White, 12 percent are Asian/Pacific Islander, 11 percent are Black, 6 percent are Hispanic, 1 percent are Native American, and 1 percent are of other racial origin. 
NMCSD Tumor Registry. The information included in this section was obtained through observations at the NMCSD Tumor Registry, by review of tumor registry certification documents, and by interviewing the NMCSD Tumor Registrar and registrar assistants.

The Tumor Registry for NMCSD is located on the medical center compound and is staffed by a certified Tumor Registrar (NTRA) and five specially trained tumor registrar assistants. The registry staff are responsible for performing a variety of technical duties necessary for the development and analysis of diagnostic and therapeutic cancer data. These duties include, but are not limited to, case finding, case accessioning, medical record abstracting, patient indexing, quality control, and patient follow-up. A brief overview of the processes supporting these duties is presented below.

Newly identified cancer cases are placed in the computerized numeric accession register, and in the alphabetic master patient index. An abstract is completed for each case, and the required follow-up data items are collected. The maximum delay for abstracting cancer cases at NMCSD is six to eight months, calculated from the date of initial diagnosis to the time the data are available for analysis. All NMCSD tumor registry abstracts are computerized. Abstract summaries and supporting medical record and follow-up data items are stored in registry files by year and accession number. Data collected by the tumor registry are used for special in-house and national studies, and are reported to the medical and administrative staff of NMCSD. A quality control plan is in place with documentation of procedures to monitor case finding, data collection, accuracy, and timeliness; and follow-up information is systematically obtained for patients in the data 
base to ensure that required follow-up rates are met. NMCSD registry data are submitted to the National Cancer Data Base (NCDB) for the purpose of continuous quality improvement of individual cancer programs, and evaluation of cancer therapies and outcomes. In addition, NMCSD cancer cases are recorded in the DoD Automated Tumor Registry (ACTUR) and state of California Tumor Registry Reporting Program (CNET). The Tumor Registry at NMCSD is accredited by the American College of Surgeons Commission on Cancer.

\section{Population and Sample}

The target population for this study was all Caucasian and African American women being diagnosed with breast cancer within the DoD MHS; and the accessible population was all Caucasian and African American women being diagnosed with breast cancer at Naval Medical Center San Diego between January 1, 1988 and December 31, 1997. The sampling design for this study was consecutive non-probability sampling (Hulley \& Cummings, 1988). The sample was composed of two comparison groups, 573 Caucasian women and 62 African American women. Even though every subject who met the selection criteria was included in this study, the minimum number of subjects (64) required in each group to conduct the $t$ test at a power of .80 , medium effect size of .50 , and significance level of .05 (Cohen, 1988) were not obtained. In addition, the assumptions for conducting the $t$ test were not met. Therefore, the Mann Whitney $U$ test was used to detect a significant difference between the two groups, in the length of time in days between discovery of breast abnormality and diagnosis of breast cancer, and in the 
length of time in days between diagnosis of breast cancer and initiation of treatment (Sheskin, 1997).

\section{Instrumentation}

The subject's tumor registry record was the primary source of data in this study. The Breast Cancer Medical Record Data Collection sheet (Appendix A) was the measurement instrument used to collect the data. In order to minimize variance and increase the precision of measurement, all data were collected by the researcher (Hulley \& Cummings, 1988), and inter-rater reliability was used to test for equivalence in collection of the data (Brink \& Wood, 1994; Polit \& Hungler, 1995). Data collection procedures and details relating to the conduction of inter-rater reliability are presented in the data collection and recording section of this chapter.

\section{Data Collection and Recording}

A list of African American and Caucasian women diagnosed with breast cancer between January 1, 1988 and December 31, 1997 was drawn from the NMCSD Tumor Board Registry computer data base. The list contained a total of 714 records and was used to select records for preliminary screening. All records on the list were initially examined by the researcher to determine eligibility for inclusion in the study. Records were selected for inclusion in the study based on the subject's race being identified as nonHispanic African American, Black, Caucasian, or White; the subject's patient status being designated as active duty, retired, or family member; diagnosis of breast cancer occurring at NMCSD or at another MHS with referral to NMCSD for treatment, and the date of diagnosis of breast cancer being documented in the tumor registry record as occurring 
between January 1, 1988 and December 31, 1997. In addition, tumor registry records were excluded from the study if the subject's record contained an Hispanic surname (maiden name), and if the diagnosis of breast cancer occurred in a civilian health care institution with referral to NMCSD after initiation of treatment. Subjects with more than one diagnosis of breast cancer appearing on the list had only the first diagnosis included in the analysis.

Finally, a total of 635 records were selected for inclusion in the study, 573 records of Caucasian women and 62 records of African American women. Identification numbers were assigned to the data collection sheets to correspond to the subject's tumor registry number, and a log of identification numbers and corresponding tumor registry record numbers was compiled and kept in a confidential file by the researcher. Names and tumor registry numbers were not included on any of the data collection tools.

Approximately three months were spent collecting the data, coding the data collection sheets, and preparing the data for statistical analysis. Data collection sheets were grouped by year and categorized within each year grouping by identification number. Each data collection sheet was reviewed for accuracy and double checked with the corresponding tumor registry record by the researcher. Double-checked data collection sheets were numerically coded by the researcher, and the numerical codes were entered into a computerized spreadsheet in preparation for statistical analysis.

Inter-rater reliability was performed by the five tumor registrar assistants on nine percent of the 635 records included in the study. Each assistant was assigned two years between 1988 and 1997, and asked to select six records for review, for each year, from 
the pre-screened list of African American and Caucasian women. Three of the 60 data collection sheets submitted by the tumor registrar assistants were excluded because the sheets reported data obtained from the tumor registry records of women with a second diagnosis of breast cancer. There were a total of 17 categories of data on each data collection sheet, resulting in a total of 969 categories for the 57 data collection sheets used for inter-rater reliability. Of the 969 categories, there were 957 matches in each category between the data recorded by the researcher and the data recorded by the tumor registrar assistants. Inter-rater reliability results indicated that the total percentage of agreement between the data recorded by the researcher and the data recorded by the tumor registrar assistants was 98.76 .

\section{Data Analysis}

The data analysis plan for this study involved descriptive and inferential statistics. Prior to statistical analyses, the data collection sheets with missing data were identified and coded (Norusis, 1997; Stevens, 1996). Missing data in the categories of marital status, service branch, and stage at diagnosis were coded as unknown and excluded from inferential statistical analysis. Missing data in the categories of tumor size, number of positive lymph nodes, and length of time between diagnosis and treatment were coded with negative numbers and excluded from inferential statistical analysis. Because of the amount of missing data within the category of rank of sponsor, missing data were coded as a separate category labeled unknown, and included in the data analysis as a separate category (Cohen \& Cohen, 1983). The categorical variables of race, rank of sponsor, and means of discovery were dummy coded for the purposes of performing multiple and 
logistic regression (Cohen \& Cohen, 1983; Daniel, 1995; Stevens, 1996; Tabachnick \& Fidell, 1996). Data analyses were performed using the Statistical Package for the Social Sciences (SPSS) for Windows. A .05 level of probability was considered to indicate statistical significance for all inferential statistical procedures.

Descriptive statistics Descriptive statistics (mean, standard deviation, minimum, maximum, frequency distributions) were utilized to summarize and describe the demographic characteristics of the sample, and the variables of the study (Hinkle, Wiersma, \& Jurs, 1994; Norusis, 1997).

Inferential statistics. The independent samples chi-square test was used to assess the relationship between each stage and age, race, socioeconomic status, and the means of discovery of breast cancer; to detect any differences in the distribution of stage at diagnosis by race; and to examine the relationship between potential (age, race, socioeconomic status, means of discovery) and realized (length of time between discovery and diagnosis, length of time between diagnosis and treatment) access (Fisher \& Van Belle, 1993; Hinkle, Wiersma \& Jurs, 1994; Huck \& Cormier, 1996; Sheskin, 1997). The Mann Whitney $U$ test was used to test for significant differences between the two groups in the length of time between discovery of breast abnormality and diagnosis, and the length of time between diagnosis and initiation of treatment (Daniel, 1995; Huck \& Cormier, 1996; Sheskin, 1997). Analysis of variance (ANOVA) was used to test for differences between the mean scores for age and the five stages of breast cancer diagnosis, within the two groups (Tabachnick \& Fidell, 1996; Hinkle, Wiersma \& Jurs, 1994). Multiple regression was used to examine the relationships between age and the length of time 
between discovery of breast abnormality and diagnosis, and the length of time between diagnosis and initiation of treatment, between the two groups; and to examine the extent to which the predictor variables of age, race, socioeconomic status, and the means of discovery of breast cancer predict utilization of breast health services (Cohen \& Cohen, 1983; Fisher \& Van Belle, 1993; Grimm \& Yarnold, 1995, Tabachnick \& Fidell, 1996). Logistic regression was used to examine the extent to which the predictor variables of age, race, socioeconomic status, and the means of discovery of breast cancer predict early and late stage at diagnosis of breast cancer; and to examine the extent to which utilization of health services predicts early and late stage at diagnosis of breast cancer (Daniel, 1995; Fisher \& Van Belle, 1993; Grimm \& Yarnold, 1995; Tabachnick \& Fidell, 1996).

\section{Limitations}

This study was limited by its methodological design in that it did not include randomized selection of subjects, control over the environment, or manipulation of the variables. Other threats to external validity, such as exclusive focus on women diagnosed at Naval Medical Center San Diego, and restriction of the race examined to African American and Caucasian, limit the generalizability of the study results. Threats to internal validity related to the measurement approach include use of the medical record, compiled and completed by various other individuals, as the source of data. In spite of these limitations, it is believed that some insight into the relationship between access and stage at diagnosis of breast cancer in African American and Caucasian women receiving care inside the MHS has been gained by conducting this study. 


\section{Human Subjects}

In order to ensure protection of each subject's freedom from intrinsic risk or injury, and to ascertain rights to privacy and dignity, a variety of human subject protective mechanisms were utilized in this study. To begin with, this proposal was submitted to both the University of San Diego and Naval Medical Center San Diego Institutional Review Boards for approval. Appendices B and C are the approval letters received from these review boards.

The Naval Medical Center San Diego Institutional Review board grants human use exemption for research involving the collection or study of existing data, documents, or records, if these sources are publicly available or if the information is recorded by the investigator in such a manner that the subjects cannot be identified directly or through identifiers linked to the subjects. In addition, the Privacy Act Statement, located in DoD healthcare records, specifically allows information to be used for research purposes. Therefore, written consent was not obtained from the subjects; however, confidentiality of the subject's data was maintained throughout the study. Identification numbers instead of names or tumor board registry numbers were attached to the individual data collection tools, and only the researcher had access to the data. Anonymity was maintained in that individual names and individual characteristics are not presented, and data analysis expresses group information, not individual information.

There were no perceived potential physical, psychological, or social risks to the subjects in this study. The findings will be used to enhance current knowledge relating to 
the relationship between access, race, and stage at diagnosis of breast cancer in women being diagnosed and treated within the DoD MHS.

\section{Summary}

The purpose of this study was to describe the relationship between access and stage at diagnosis in African American and Caucasian women being diagnosed with breast cancer within the DoD MHS. The data analysis plan described above is felt to have been appropriate for the study's purpose and methodology. It is hoped that this project has provided insight into the role of access and its influence on participation in breast health care programs and early diagnosis of breast cancer. 


\section{CHAPTER IV}

\section{RESULTS}

The results of the data analysis are presented in four sections in this chapter. Section one presents a description of the sample. Section two presents comparative descriptive analysis of the study variables and selected demographic characteristics; and section three presents the statistical analysis related to each research question. Section four presents the results from supplemental data analysis.

\section{Description of the Sample}

Table 5 provides a demographic profile of the subjects included in the study. Sixty two of the women were African American (9.8\%) and 573 (90.2\%) were Caucasian. The majority of the women were family members of active duty or retired service members (93.4\%). Active duty women constituted 4.7 percent of the sample, and retired women constituted 1.9 percent. Of the 635 women included in the analysis 78.9 percent were married, and 70 percent were affiliated with the United States Navy. The largest rank of sponsor grouping was E-7 (17.8\%), followed by the unknown grouping in which the sponsor's rank could not be identified from the tumor registry record (16.1\%). The mean age of the sample was $58.03(\mathrm{SD}=13.57)$.

The majority of the women included in the study were diagnosed at stage 1 ( $n=$ 274 or $43.1 \%)$. Stage 4 formed the smallest stage at diagnosis grouping $(n=29$ or $4.6 \%)$, and 2.8 percent $(n=18)$ of the tumor registry records analyzed did not have a 
Table 5

Summary of Demographic and Study Variable Characteristics

\begin{tabular}{lcr} 
Characteristic & No. of cases & $\%$ of total \\
\hline Women with breast cancer & 573 & 90.2 \\
Caucasian & 62 & 9.8
\end{tabular}

Patient service status

Family member

Active duty

Retired

12

Marital status

Married

Widowed

101

15.9

Single

Divorced

11

1.7

Separated

5

0.8

Unknown

2

0.3

Branch of service affiliation

Navy

Marine Corps

79

12.4

Air Force

55

8.7

(table continues) 


\begin{tabular}{|c|c|c|}
\hline Characteristic & No. of cases & $\%$ of total \\
\hline Army & 36 & 5.7 \\
\hline Unknown & 15 & 2.4 \\
\hline Other & 3 & 0.5 \\
\hline Coast Guard & 2 & 0.3 \\
\hline \multicolumn{3}{|l|}{ Rank of sponsor } \\
\hline $\mathrm{E}-7$ & 113 & 17.8 \\
\hline Unknown & 102 & 16.1 \\
\hline E-6 & 91 & 14.3 \\
\hline $0-5$ & 53 & 8.3 \\
\hline $0-6$ & 47 & 7.4 \\
\hline E-8 & 46 & 7.2 \\
\hline $\mathrm{O}-4$ & 38 & 6.0 \\
\hline E-9 & 34 & 5.4 \\
\hline $0-3$ & 34 & 5.4 \\
\hline E-5 & 31 & 4.9 \\
\hline CWO & 23 & 3.6 \\
\hline E-4 & 9 & 1.4 \\
\hline $0-7$ & 4 & 0.6 \\
\hline $0-8$ & 4 & 0.6 \\
\hline
\end{tabular}

(table continues) 


\begin{tabular}{ccr}
\hline Characteristic & No. of cases & $\%$ of total \\
\hline O-2 & 3 & 0.5 \\
E-1 & 1 & 0.2 \\
O-9 & 1 & 0.2 \\
O-10 & 1 & 0.2
\end{tabular}

Breast cancer diagnosis by year

$\begin{array}{lll}1988 & 39 & 6.1 \\ 1989 & 55 & 8.7 \\ 1990 & 62 & 9.8 \\ 1991 & 65 & 10.2 \\ 1992 & 75 & 11.8 \\ 1993 & 71 & 11.2 \\ 1994 & 53 & 8.3 \\ 1995 & 68 & 10.7 \\ 1996 & 71 & 11.2 \\ 1997 & 76 & 12.0\end{array}$

Age at diagnosis $(\underline{N}=635)$

M: $\quad 58.03$

SD: $\quad 13.57$

minimum: 25

maximum: 97

(table continues) 


\begin{tabular}{ccr}
\hline Characteristic & No. of cases & \% of total \\
\hline Stage at diagnosis & 274 & 43.1 \\
Stage 1 & 183 & 28.8 \\
Stage 2 & 76 & 12.0 \\
In Situ & 55 & 8.7 \\
Stage 3 & 29 & 4.6 \\
Stage 4 & 18 & 2.8 \\
Not staged & 18
\end{tabular}

Tumor size in centimeters $(\underline{N}=567)$

M: 2.2

SD: 1.86

minimum: 0

maximum: 13

Number of positive lymph nodes $(\underline{N}=508)$

M: 2.07

$\underline{\text { SD: }} 4.69$

minimum: 0

maximum: 37

Means of discovery

$\begin{array}{lll}\text { Routine mammogram } & 288 & 45.4\end{array}$

$\begin{array}{lll}\text { Incidental BSE } & 195 & 30.7\end{array}$

$\begin{array}{lll}\text { Intentional BSE } & 62 & 9.8\end{array}$

$\begin{array}{lll}\text { Routine CBE } & 37 & 5.8\end{array}$

(table continues) 


\begin{tabular}{lcr}
\hline Characteristic & No. of cases & $\%$ of total \\
\hline Secondary CBE & 25 & 3.9 \\
Secondary Mammogram & 17 & 2.7 \\
Unknown & 11 & 1.7
\end{tabular}

Length of time (in days) between discovery and diagnosis

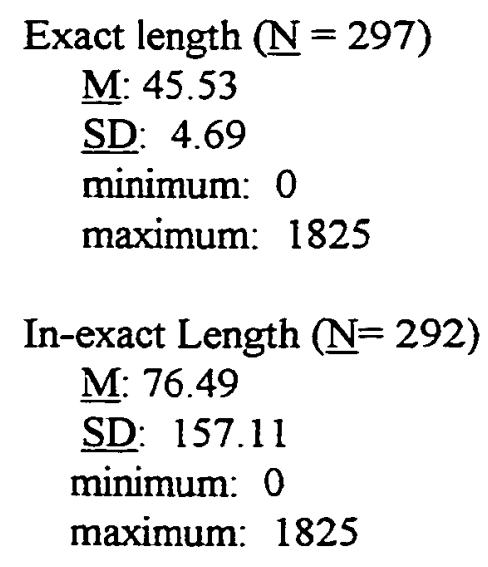

Length of time (in categories) between discovery and diagnosis

$\begin{array}{lcr}15-30 \text { days } & 138 & 21.7 \\ 31-60 \text { days } & 116 & 18.3 \\ <7 \text { days } & 104 & 16.4 \\ 7-14 \text { days } & 98 & 15.4 \\ \text { Unknown } & 47 & 7.4 \\ 61-90 \text { days } & 38 & 6.0 \\ & & \text { (table continues) }\end{array}$




\section{Characteristic}

$$
91 \text { - } 120 \text { days }
$$

$121-240$ days

$>240$ days

Length of time (in days) between diagnosis

and treatment $(\underline{\mathrm{N}}=629)$

M: 14.43

SD: 18.41

minimum: 0

maximum: 180

Means of initial treatment

Surgery after diagnosis

Surgery, concurrent with diagnosis

Chemotherapy

Radiation therapy

9

1.4

No treatment

Hormone therapy

Survival status at the time of data collection

Alive

536

84.4

Deceased, breast cancer

8.0

Deceased, unknown

Deceased, other cause 
stage at diagnosis recorded. The mean tumor size was 2.2 centimeters $(\mathrm{SD}=1.86)$, and the mean number of positive lymph nodes examined was $2.07(\mathrm{SD}=4.69)$.

Forty-five percent of the breast cancer cases were discovered by mammogram $(n=$ 288 ), followed by incidental BSE ( $n=195$ or $30.7 \%$ ). The means of discovery could not be determined from the tumor registry record in 1.7 percent of the cases. The length of time between discovery of a breast abnormality and diagnosis of breast cancer could not be calculated by examining the exact date of discovery in 292 of the cases. In these cases, the in-exact length of time in days was calculated by counting the number of days between the 15 th of the month in which the breast abnormality was discovered and the exact date of diagnosis. The mean in-exact length of time between discovery and diagnosis $(\mathrm{N}=$ 292) was 76.49 days $(S D=157.11)$. The mean exact length of time in days between discovery and diagnosis $(\mathrm{N}=297)$ was $45.53(\mathrm{SD}=4.69)$. The length of time between discovery and diagnosis could not be identified from the tumor registry record in 46 of the cases. Twenty-two percent of the cases included in the analysis had a length of time between discovery and diagnosis which fell into the 15 to 30 day range, 18.3 percent fell into the 31 to 60 day range, 14.4 percent had less than 7 days between discovery and diagnosis, and 20.9 percent of the cases fell into the greater than 60 day range. The mean length of time in days between diagnosis and treatment was $14.43(\mathrm{SD}=18.41)$.

Surgery after diagnosis was the most frequently used means of initial treatment (n $=343$ or $54 \%)$, and $40.3 \%(n=256)$ of the women included in the study were treated initially with surgery concurrent with diagnosis. Chemotherapy was the means of initial treatment in 3.1 percent $(n=20)$ of the cases, and 1.4 percent $(n=9)$ of the women were 
treated initially with radiation therapy. At the time of data collection, the tumor registry records indicated that 84.8 percent $(n=536)$ of the women included in the study were alive, 8 percent $(n=51)$ were deceased due to breast cancer, and 7.5 percent were deceased due to other $(n=11)$ or unknown causes $(n=37)$.

Descriptive Analysis of the Study Variables

Comparative descriptive statistics of the study variables and selected demographic characteristics of the African American and Caucasian women, included in the study are presented in Table 6. T tests, Mann Whitney $\mathrm{U}$ tests, and independent samples chi-square tests were used to examine differences in means and frequency distributions.

There was a significant difference in the mean ages for African American ( $M=$ 47.94) and Caucasian women $(M=59.13)(p=.000)$. Twenty-nine percent of the African American women diagnosed with breast cancer were less than 40 years old, compared to 8.7 percent of the Caucasian women; and 55.5 percent of the Caucasian women diagnosed with breast cancer were 60 or older as compared to 19.3 percent of the African American women.

There was no significant difference between the two groups on marital status $(p=$ $.495)$, but the groups differed significantly on patient service status $(p=.000)$. Sixteen percent of the African American women were active duty, compared to 3.5 percent of the Caucasian women, and 94.8 percent of the Caucasian women were family members, compared to 80.6 percent of the African American women. The two groups also differed significantly on the rank of the sponsor $(p=.000)$. Twenty-eight percent of the African American women fell into the E1 to E5 category as compared to 5.8 percent of the 
Table 6

Between Group Differences in Distribution of Study Variables

\begin{tabular}{|c|c|c|c|}
\hline Variable & $\begin{array}{l}\text { African } \\
\text { American }\end{array}$ & Caucasian & $p$ value \\
\hline \multicolumn{4}{|c|}{ Age (years), $\%$ (no. of cases) } \\
\hline$<40$ & $29 \quad(18)$ & $8.7 \quad(50)$ & \\
\hline $40-49$ & 24.2 (15) & $17.3 \quad(99)$ & \\
\hline $50-59$ & 27.4 (17) & $18.5(106)$ & \\
\hline $60-69$ & $14.5 \quad(9)$ & (193) & \\
\hline$>=70$ & $4.8 \quad(3)$ & $(125)$ & $.000^{*}$ \\
\hline \multicolumn{4}{|c|}{ Marital status, \% (no. of cases) } \\
\hline Married & $75.8(47)$ & $79.5(454)$ & \\
\hline Unmarried & $24.2(15)$ & $20.5(117)$ & .495 \\
\hline \multicolumn{4}{|c|}{ Patient service status, $\%$ (no. of cases) } \\
\hline Active duty & $16.1(10)$ & $3.5(20)$ & \\
\hline Retired & $3.2 \quad(2)$ & $1.7(10)$ & \\
\hline Family member & $80.6(50)$ & $94.8(543)$ & $.000^{*}$ \\
\hline \multicolumn{4}{|c|}{ Rank of sponsor, \% (no. of cases) } \\
\hline $\mathrm{E} 1-\mathrm{E} 5$ & $27.7(13)$ & $5.8(28)$ & \\
\hline E6 - E9 & $63.8(30)$ & $52.3(254)$ & \\
\hline CWO - O4 & $4.3(2)$ & $19.8(96)$ & \\
\hline $05-010$ & $4.3(2)$ & $22.2(108)$ & $000^{*}$ \\
\hline
\end{tabular}




\begin{tabular}{clll}
\hline Variable & $\begin{array}{l}\text { African } \\
\text { American }\end{array}$ & Caucasian & p value \\
\hline Stage at diagnosis, \% (no. of cases) & $14.8(9)$ & $12.1(67)$ \\
In Situ & $26.2(16)$ & $46.4(258)$ \\
Stage 1 & $42.6(26)$ & $28.2(157)$ \\
Stage 2 & $13.1(8)$ & $8.5(47)$ \\
Stage 3 & $3.3(2)$ & $4.9(27)$ \\
Stage 4 & $18.5(10)$ & $27.9(143)$ \\
Tumor size, \% (no. of cases) & $27.8(15)$ & $38.4(197)$ \\
$0-1 \mathrm{~cm}$ & $27.8(15)$ & $17.2(88)$ \\
$1.1-2.0 \mathrm{~cm}$ & $18.5(10)$ & $9.2(47)$ \\
$2.1-3.0 \mathrm{~cm}$ & $7.4(4)$ & $7.4(38)$ \\
$3.1-5.0 \mathrm{~cm}$ & & $029 *$ \\
$5.0 \mathrm{~cm}$ & &
\end{tabular}

No. of lymph nodes, \% (no. of cases)

0

1-3

$4-10$

$>10$

Means of discovery, \% (no. of cases)

Intentional BSE

Incidental BSE
$58.3(28) \quad 66.5(306)$

$22.9(11) \quad 14.3(66)$

$10.4(5) \quad 13.5(62)$

$8.3(4) \quad 5.7(26)$

.336 


\begin{tabular}{|c|c|c|c|}
\hline Variable & $\begin{array}{l}\text { African } \\
\text { American }\end{array}$ & Caucasian & $\mathrm{p}$ value \\
\hline $\mathrm{CBE}$ & $15.8(9)$ & $9.3(53)$ & \\
\hline Mammogram & $21.1(12)$ & $51.7(293)$ & $.000^{*}$ \\
\hline \multicolumn{4}{|l|}{$\begin{array}{l}\text { Means of initial treatment } \\
\% \text { (no. of cases) }\end{array}$} \\
\hline $\begin{array}{l}\text { Surgery concurrent with } \\
\text { diagnosis }\end{array}$ & $37.1(23)$ & $41.0(233)$ & \\
\hline Surgery after diagnosis & $61.3(38)$ & $53.7(305)$ & \\
\hline Other treatment & $1.6(1)$ & $5.3(30)$ & .311 \\
\hline \multicolumn{4}{|l|}{$\begin{array}{l}\text { Survival status at the time of data } \\
\text { collection, } \% \text { (no. of cases) }\end{array}$} \\
\hline Alive & $77.4(48)$ & $85.2(488)$ & \\
\hline Deceased, Breast Cancer & $16.1(10)$ & $7.2(41)$ & \\
\hline Deceased (Unknown, Other) & $6.5(4)$ & 7.7 (44) & $.047 * *$ \\
\hline
\end{tabular}

Note. All $\mathrm{p}$ values are 2 sided. P values were derived from chi-square tests.

Other treatment includes chemotherapy, radiation therapy, and hormone therapy.

* significant at $\mathrm{p}<.001 \quad * *$ significant at $\mathrm{p}<.05$ 
Caucasian women; 63.8 percent of the African American women fell into the E6 to E 9 category, compared to 52.3 percent of the Caucasian women; 19.8 percent of the Caucasian women fell into the CWO - O4 category compared to 4.3 percent of the African women; and 22.2 percent of the Caucasian women fell into the $\mathrm{O} 5$ to $\mathrm{O} 10$ category, compared to 4.3 percent of the African American women.

The two groups differed significantly on stage at diagnosis of breast cancer $(p=$ $.029)$, tumor size $(p=.035)$, and means of discovery $(p=.000)$. But there were no significant differences between the two groups on number of positive lymph nodes examined $(p=.336)$ or means of initial treatment $(p=.311)$. At the time of data collection, tumor registry records reflected that 16.1 percent of the African American women $(n=10)$ were deceased due to breast cancer as compared to 7.2 percent of the Caucasian women $(n=41)$.

The mean exact length of time in days between discovery and diagnosis of breast cancer was 45.15 for African American women $(n=20)$ and 45.55 for Caucasian women $(n=277)$. The mean in-exact length of time between discovery and diagnosis was 65.08 for African American women $(n=36)$ and 78.09 for Caucasian women $(n=256)$; and the combined exact and in-exact length of time between discovery and diagnosis was 57.96 for African American women and 61.29 for Caucasian women. The mean length of time in days between diagnosis and initiation of treatment was 13.26 for African American women $(n=62)$ and 14.56 for Caucasian women $(n=567)$. Because of sample size and missing data on the length of time between discovery and diagnosis, and the length of time between diagnosis and treatment variables, the minimum number of subjects (64) required 
in each group to conduct the $t$ test to detect differences between the two groups at a power of .80 , medium effect size of, 50 and .05 significance level using Cohen's method, was not obtained. Therefore, the Mann Whitney test was used to detect differences between the African American and Caucasian women included in the study, on length of time between discovery and diagnosis, and length of time between diagnosis and treatment. There were no significant differences between the two groups on length of time in days between discovery and diagnosis $(U=14296, p=.620$, two tailed $)$ or length of time in days between diagnosis and treatment $(U=17576.500, p=1.000$ two tailed).

Data Analysis Related to the Research Questions

Research Question 1: What are the relationships between age, race, socioeconomic status, the means of discovery of breast cancer, and stage at diagnosis of breast cancer in women identifying their race as Caucasian or African American, and being diagnosed with breast cancer at Naval Medical Center San Diego from January 1, 1988 to December 31, 1997 ? Independent samples chi-square tests, to assess the relationship between each stage and age, race, socioeconomic status, and the means of discovery of breast cancer, and to detect any differences in distribution by race; and one-way analysis of variance to test for differences between the mean scores for the five stages of breast cancer diagnosis between the two groups, were obtained using the Statistical Package for the Social Sciences (SPSS) for Windows.

Table 7 shows that a significant relationship was found between age at diagnosis and each stage, $X^{2}(16, N=617)=44.202, p=.000$. Any standardized residual with an 
Table 7

Relationship Between Stage at Diagnosis and Age at Diagnosis

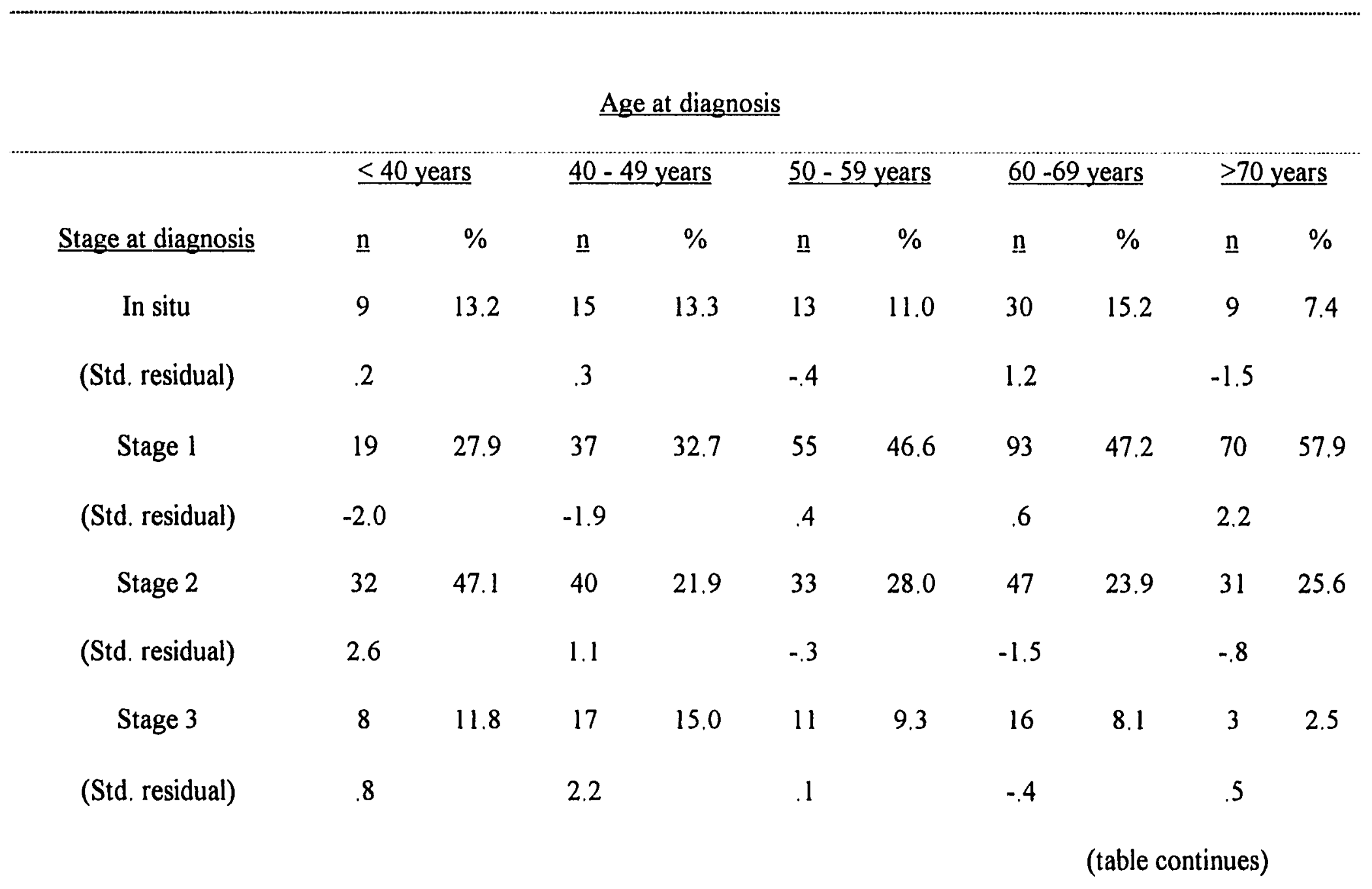




\begin{tabular}{|c|c|c|c|c|c|c|c|c|c|c|}
\hline \multirow[b]{3}{*}{ Stage at diagnosis } & \multicolumn{6}{|c|}{ Age at diagnosis } & & & & \\
\hline & \multicolumn{2}{|c|}{$\leq 40$ years } & \multicolumn{2}{|c|}{$\underline{40-49 \text { years }}$} & \multicolumn{2}{|c|}{$\underline{50-59 \text { years }}$} & \multicolumn{2}{|c|}{$60-69$ years } & \multicolumn{2}{|c|}{$\geq 70$ years } \\
\hline & $\underline{\mathrm{n}}$ & $\%$ & $\underline{\mathrm{n}}$ & $\%$ & $\underline{\mathrm{n}}$ & $\%$ & $\underline{\mathrm{n}}$ & $\%$ & $\underline{\mathrm{n}}$ & $\%$ \\
\hline Stage 4 & 0 & 0 & 4 & 3.5 & 6 & 5.1 & 11 & 5.6 & 8 & 6.6 \\
\hline (Std. residual) & -1.8 & & -.6 & & .2 & & .6 & & 1.0 & \\
\hline Total & 68 & 100 & 113 & 100 & 118 & 100 & 197 & 100 & 121 & 100 \\
\hline
\end{tabular}

Note. $X^{2}(16, N=617)=44.202, p=.000$ 
absolute value greater than or equal to the tabled critical two-tailed .05 value $z .05=1.96$ is significant at the .05 level and contributes significantly to the obtained chi-square value (Sheskin, 1997). Based on the computation of standardized residuals, the observed frequency of women less than 40 years old, diagnosed at stage $2(47.1 \%$, std. residual $=$ 2.6), women $40-49$ years old diagnosed at stage $3(15 \%$, std. residual $=2.2)$, and women less than 40 years old diagnosed at stage $1(27.9 \%$, std. residual $=-2.0)$ differed significantly from the expected frequency, and contributed significantly to the obtained chi-square value.

Table 8 shows that there were no significant differences between the mean scores for age and the five stages of breast cancer for African American women, $F=1.058, p=$ .386 ; however, there were significant differences detected in the group of Caucasian women, $F=5.589, p=.000$. Table 9 displays further analysis of the relationship between stage at diagnosis and age, and shows the distribution of age by early (in situ, stage 1) and late (stage 2,3,4) stage at diagnosis, for African American and Caucasian women. In support of the data analysis reported in Table 8, Table 9 shows that the distribution of age by early-late stage did not differ in the African American women included in the study, $\mathrm{X}^{2}$ $(2, N=61)=4.331, p=.115$. The significant difference in distribution of age by stage in the Caucasian women, $X^{2}(2, N=556)=14.902, p=.001$, was contributed to by the observed frequency of Caucasian women less than 40 and diagnosed in late stage breast cancer $(14.7 \%$, std. residual $=2.1)$ differing significantly from the expected frequency. As shown in Table 10, there was a significant relationship between race and stage at diagnosis, $X^{2}(4, N=617)=10.83, p=.029$. The observed frequency of African American women diagnosed at stage $1(26.2 \%$, std. residual $=-2.1)$ differed significantly 
Table 8

One-Way Analysis of Variance: Mean Age at Diagnosis and Stage at Diagnosis, by Race

\begin{tabular}{|c|c|c|c|c|c|}
\hline Source & $\underline{\mathrm{SS}}$ & df & $\underline{\mathrm{MS}}$ & $\underline{F}$ & $\mathrm{p}$ value \\
\hline \multicolumn{6}{|c|}{ African American $(n=61)$} \\
\hline Between Groups & 624.259 & 4 & 156.065 & 1.058 & .386 \\
\hline Within Groups & 8261.184 & 56 & 147.521 & & \\
\hline Total & 8885.443 & 60 & & & \\
\hline \multicolumn{6}{|c|}{ Caucasian $(n=556)$} \\
\hline Between Groups & 3775.543 & 4 & 943.886 & 5.589 & $.000^{*}$ \\
\hline Within Groups & 93051.090 & 551 & 168.877 & & \\
\hline Total & 96826.633 & 555 & & & \\
\hline
\end{tabular}

Note. * significant at_p $<.05$ 
Table 9

Relationship Between Stage at Diagnosis and Age at Diagnosis, by Race

\begin{tabular}{|c|c|c|c|c|}
\hline \multirow[b]{3}{*}{ Age at diagnosis } & \multicolumn{4}{|c|}{ Stage } \\
\hline & \multicolumn{2}{|c|}{ Early (In Situ, Stage 1) } & \multicolumn{2}{|c|}{ Late (Stage 2, 3, 4) } \\
\hline & $\underline{\mathbf{n}}$ & $\%$ & $\underline{\mathbf{n}}$ & $\%$ \\
\hline \multicolumn{5}{|c|}{ African American } \\
\hline$<=40$ & 9 & 36.0 & 10 & 27.8 \\
\hline (Std. residual) & .4 & & -.4 & \\
\hline $41-60$ & 10 & 40.0 & 23 & 63.9 \\
\hline (Std. residual) & -1.0 & & .8 & \\
\hline$>=61$ & 6 & 24 & 3 & 8.3 \\
\hline (Std. residual) & 1.2 & & -1.0 & \\
\hline Total & 25 & 100 & 36 & 100 \\
\hline \multicolumn{5}{|c|}{ Caucasian $^{\mathrm{b}}$} \\
\hline$<=40$ & 23 & 7.1 & 34 & 14.7 \\
\hline (Std. residual) & -1.8 & & 2.1 & \\
\hline $4 l-60$ & 117 & 36 & 99 & 42.9 \\
\hline (Std. residual) & -.8 & & 1.0 & \\
\hline$>=61$ & 185 & 56.9 & 98 & 42.4 \\
\hline (Std. residual) & 1.5 & & -1.8 & \\
\hline Total & 325 & 100 & 231 & 100 \\
\hline
\end{tabular}

Note. ${ }^{a} X^{2}(2, N=61)=4.331, \underline{p}=.115$

${ }^{b} X^{2}(2, N=556)=14.902, \underline{p}=.001$ 
Table 10

Relationship Between Stage at Diagnosis and Race

\begin{tabular}{|c|c|c|c|c|}
\hline \multirow[b]{3}{*}{ Stage at diagnosis } & \multicolumn{4}{|c|}{ Race } \\
\hline & \multicolumn{2}{|c|}{ African American } & \multicolumn{2}{|c|}{ Caucasian } \\
\hline & $\underline{\mathbf{n}}$ & $\%$ & $\underline{\mathbf{n}}$ & $\%$ \\
\hline In situ & 9 & 14.8 & 67 & 12.1 \\
\hline (Std. residual) & .5 & & -.2 & \\
\hline Stage 1 & 16 & 26.2 & 258 & 46.4 \\
\hline (Std. residual) & -2.1 & & .7 & \\
\hline Stage 2 & 26 & 42.6 & 157 & 28.2 \\
\hline (Std. residual) & 1.9 & & -.6 & \\
\hline Stage 3 & 8 & 13.1 & 47 & 8.5 \\
\hline (Std. residual) & 1.1 & & -.4 & \\
\hline Stage 4 & 2 & 3.3 & 27 & 4.9 \\
\hline (Std. residual) & -.5 & & .2 & \\
\hline Total & 61 & 100 & 556 & 100 \\
\hline
\end{tabular}

Note. $X^{2}(4, N=617)=10.83, \underline{p}=.029$ 
from the expected frequency, and contributed significantly to the obtained chi-square value. Table 11 shows a significant relationship between stage at diagnosis and socioeconomic status (rank of sponsor), $\mathrm{X}^{2}(12, \mathrm{~N}=519)=26.72, \mathrm{p}=.008$. The obtained chi-square value was contributed to significantly by the observed frequency of women with a sponsor's rank of El - E5 and diagnosed at stage $2(53.8 \%$, std. residual $=$ 2.7) differing significantly from the expected frequency.

Table 12 shows that a significant relationship was found between stage at diagnosis of breast cancer and the means of discovery, $X^{2}(12, N=606)=134.46, p=$ .000 . The observed frequency of women with breast cancer discovered by mammogram and diagnosed at stage $2(15.8 \%$, std. residual $=-4.4)$, by mammogram and diagnosed at in situ $(20.9 \%$, std. residual $=4.2)$, by incidental BSE and diagnosed at stage $2(44.5 \%$, std. residual $=3.8)$, by CBE and diagnosed at stage $4(15.5 \%$, std. residual $=3.7)$, by incidental BSE and diagnosed at in situ $(3.7 \%$, std. residual $=-3.4)$, by mammogram and diagnosed at stage $1(57.2 \%$, std. residual $=3.4)$, and by incidental BSE and diagnosed at stage $1(21.3 \%$, std. residual $=-3.0)$, differed significantly from the expected frequency and contributed significantly to the obtained chi-square value.

The distribution of rank of sponsor (see Table 13) for African American women was significantly different from the distribution for Caucasian women, $X^{2}(3, N=533)=$ $40.09, \mathrm{p}=.000$ ). The observed frequency of African American women with a sponsor's rank of El $-\mathrm{E} 5(27.7 \%$, std. residual $=4.9), \mathrm{CWO}-\mathrm{O} 4(4.3 \%$, std. residual $=-2.3)$, and $05-O 10(4.3 \%$, std. residual $=-2.5)$ differed significantly from the expected frequency and contributed significantly to the obtained chi-square value. Table 14 shows that the distribution of the means of discovery for African American women was significantly 
Table 11

$\underline{\text { Relationship Between Stage at Diagnosis and Rank of Sponsor }}$

\begin{tabular}{|c|c|c|c|c|c|c|c|c|}
\hline \multicolumn{9}{|c|}{ Rank of Sponsor } \\
\hline & \multicolumn{2}{|c|}{$E 1-E 5$} & \multicolumn{2}{|c|}{$\underline{E 6-E 9}$} & \multicolumn{2}{|c|}{ CWO - 04} & \multicolumn{2}{|c|}{$\underline{05-010}$} \\
\hline Stage at diagnosis & $\underline{\mathrm{n}}$ & $\%$ & $\underline{n}$ & $\%$ & $\underline{\mathrm{n}}$ & $\%$ & $\underline{\mathbf{n}}$ & $\%$ \\
\hline In situ & 2 & 5.1 & 32 & 11.5 & 12 & 12.6 & 16 & 15.1 \\
\hline (Std. residual) & -1.2 & & -.2 & & .2 & & .9 & \\
\hline Stage 1 & 10 & 25.6 & 122 & 43.7 & 43 & 45.3 & 58 & 54.7 \\
\hline (Std. residual) & -1.8 & & -.3 & & .1 & & 1.5 & \\
\hline Stage 2 & 21 & 53.8 & 82 & 29.4 & 31 & 32.6 & 23 & 21.7 \\
\hline (Std. residual) & 2.7 & & -.3 & & .4 & & -1.6 & \\
\hline Stage 3 & 6 & 15.4 & 27 & 9.7 & 5 & 5.3 & 7 & 6.6 \\
\hline (Std , residual) & 1.4 & & 6 & & -1.1 & & -.7 & \\
\hline
\end{tabular}




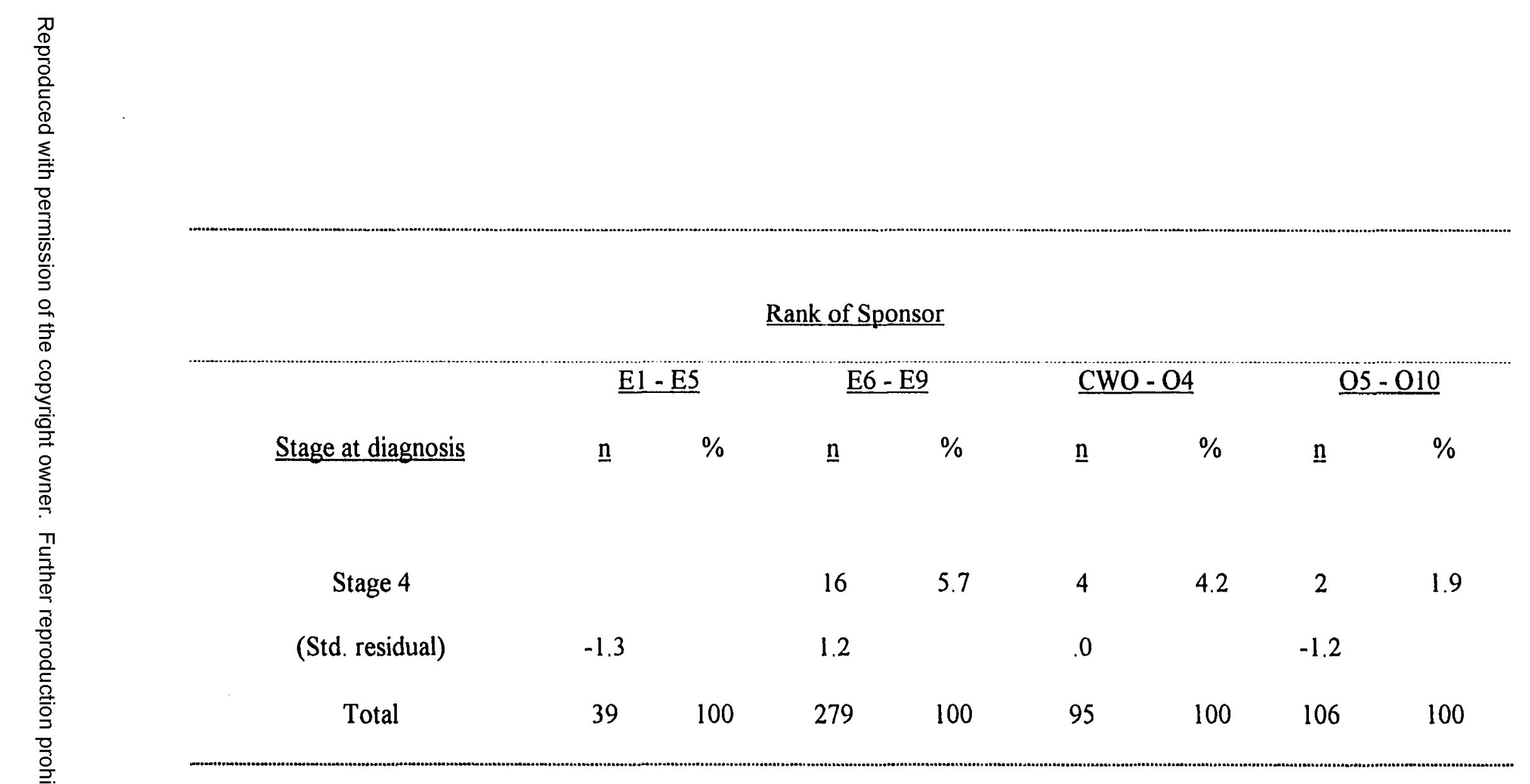

Note. $X^{2}(12, N=519)=26.72, p=.008$

Five cells $(25 \%)$ have an expected count of less than 5 . The minimum expected frequency is 1.65 . 
Table 12

Relationship Between Stage at Diagnosis and Means of Discovery

\begin{tabular}{|c|c|c|c|c|c|c|c|c|}
\hline \multicolumn{9}{|c|}{ Means of discovery } \\
\hline \multirow[b]{2}{*}{ Stage at diagnosis } & \multicolumn{2}{|c|}{ Intentional BSE } & \multicolumn{2}{|c|}{ Incidental BSE } & \multicolumn{2}{|c|}{$\mathrm{CBE}$} & \multicolumn{2}{|c|}{ Mammogram } \\
\hline & $\underline{\mathrm{n}}$ & $\%$ & $\underline{\mathrm{n}}$ & $\%$ & $\underline{\mathrm{n}}$ & $\%$ & $\underline{\mathrm{n}}$ & $\%$ \\
\hline In situ & 4 & 5 & 7 & 3.7 & 3 & 5.2 & 62 & 20.9 \\
\hline (Std. residual) & -1.6 & & -3.4 & & -1.6 & & 4.2 & \\
\hline Stage 1 & 20 & 33.3 & 57 & 21.3 & 21 & 36.2 & 170 & 57.2 \\
\hline (Std. residual) & -1.3 & & -3.0 & & -.9 & & 3.4 & \\
\hline Stage 2 & 27 & 45 & 85 & 44.5 & 21 & 36.2 & 47 & 15.8 \\
\hline (Std. residual) & 2.2 & & 3.8 & & .9 & & -4.4 & \\
\hline Stage 3 & 8 & 13.3 & 29 & 15.2 & 4 & 6.9 & 13 & 4.4 \\
\hline (Std. residual) & 1.1 & & 2.9 & & -.5 & & -2.6 & \\
\hline
\end{tabular}




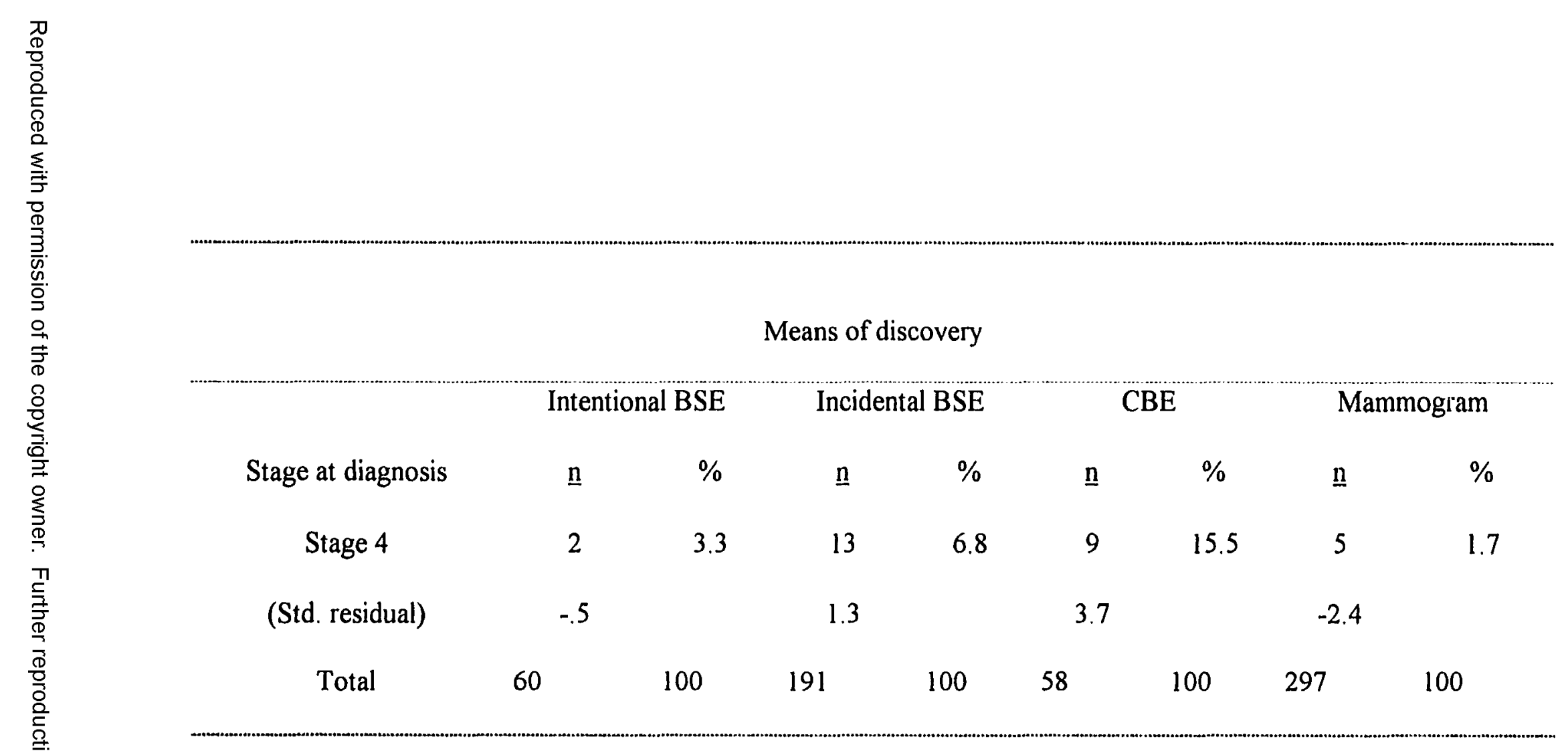

Note. $X^{2}(12, N=606)=134.46, p=.000$ 
Table 13

Distribution of Rank of Sponsor By Race

\begin{tabular}{|c|c|c|c|c|}
\hline \multirow[b]{3}{*}{ Rank of sponsor } & \multicolumn{2}{|c|}{ Race } & \multirow{2}{*}{\multicolumn{2}{|c|}{ Caucasian }} \\
\hline & \multicolumn{2}{|c|}{ African American } & & \\
\hline & $\underline{\mathbf{n}}$ & $\%$ & $\underline{\mathbf{n}}$ & $\%$ \\
\hline $\mathrm{E} 1-\mathrm{E} 5$ & 13 & 27.7 & 28 & 5.8 \\
\hline (Std. residual) & 4.9 & & -1.5 & \\
\hline E6 - E9 & 30 & 63.8 & 254 & 52.3 \\
\hline (Std. residual) & 1.0 & & -.3 & \\
\hline CWO - O4 & 2 & 4.3 & 96 & 19.8 \\
\hline (Std. residual) & -2.3 & & .7 & \\
\hline $05-010$ & 2 & 4.3 & 108 & 22.2 \\
\hline (Std. residual) & -2.5 & & .8 & \\
\hline Total & 47 & 100 & 486 & 100 \\
\hline
\end{tabular}

Note. $X^{2}(3, \mathrm{~N}=533)=40.09, \underline{p}=.000$ 
Table 14

Distribution of Means of Discovery, By Race

\begin{tabular}{|c|c|c|c|c|}
\hline \multirow[b]{3}{*}{ Means of discovery } & \multicolumn{3}{|c|}{ Race } & \\
\hline & Africa & ican & \multicolumn{2}{|c|}{ Caucasian } \\
\hline & $\underline{\mathrm{n}}$ & $\%$ & $\underline{\mathbf{n}}$ & $\%$ \\
\hline Intentional BSE & 10 & 17.5 & 52 & 9.2 \\
\hline (Std. residual) & 1.8 & & -.6 & \\
\hline Incidental BSE & 26 & 45.6 & 169 & 29.8 \\
\hline (Std. residual) & 1.9 & & -.6 & \\
\hline CBE & 9 & 15.8 & 53 & 9.3 \\
\hline (Std. residual) & 1.4 & & -.4 & \\
\hline Mammogram & 12 & 21.1 & 293 & 51.7 \\
\hline (Std. residual) & -3.0 & & 1.0 & \\
\hline Total & 57 & 100 & 567 & 100 \\
\hline
\end{tabular}

Note. $\left.\quad X^{2}(3, N=624)=19.90, p=.000\right)$ 
different from the distribution for Caucasian women $\left(X^{2}(3, N=624)=19.90, p=.000\right)$. The observed frequency of African American women with breast cancer discovered by mammogram $(21.1 \%$, std. residual $=-3.0)$ differed significantly from the expected frequency and contributed significantly to the obtained chi-square value.

Research Question 2: What are the relationships between the length of time between discovery and diagnosis of breast cancer, and the length of time between diagnosis and initiation of treatment of breast cancer, and stage at diagnosis of breast cancer in women identifying their race as Caucasian or African American, and being diagnosed with breast cancer at Naval Medical Center San Diego from January 1, 1988 to December 31, 1997 ? Independent samples chi-square tests to assess the relationship between each stage and the length of time between discovery and diagnosis, and the length of time between diagnosis and treatment were obtained using SPSS.

Table 15 shows that a significant relationship was found between the length of time between discovery and diagnosis and stage at diagnosis of breast cancer, $X^{2}(16, N=$ $572)=35.834, p=.003$. The observed frequency of breast cancer cases diagnosed at stage 3 and having greater than 91 days between discovery and diagnosis $(18.9 \%$, std. residual $=3.2$ ), and at stage 4 and having 61 to 90 days between discovery and diagnosis, differed significantly from the expected frequency and contributed significantly to the obtained chi-square value.

As shown in Table 16, there was also a significant relationship between stage at diagnosis and the length of time between diagnosis and treatment, $X^{2}(12, N=617)=$ $53.330, p=.000$. Length of time in days between diagnosis and treatment categories were compressed to ensure that no more than 25 percent of the cells had expected frequency 
Table 15

Relationship Between Stage at Diagnosis and Length of Time Between Discovery and Diagnosis

\begin{tabular}{|c|c|c|c|c|c|c|c|c|c|c|}
\hline \multicolumn{11}{|c|}{ Length of time between discovery and diagnosis } \\
\hline & \multicolumn{2}{|c|}{$\underline{0-14 \text { days }}$} & \multicolumn{2}{|c|}{$15-30$ days } & \multicolumn{2}{|c|}{$\underline{31-60 \text { days }}$} & \multicolumn{2}{|c|}{$61-90$ days } & \multicolumn{2}{|c|}{$\geq 91$ days } \\
\hline Stage at diagnosis & $\underline{\mathrm{n}}$ & $\%$ & $\underline{\mathrm{n}}$ & $\%$ & $\underline{\mathrm{n}}$ & $\%$ & $\underline{\mathrm{n}}$ & $\%$ & $\underline{\mathrm{n}}$ & $\%$ \\
\hline In situ & 25 & 12.5 & 17 & 12.6 & 18 & 16.2 & 3 & 8.3 & 7 & 7.8 \\
\hline (Std. residual) & .1 & & .1 & & 1.2 & & .5 & & -1.2 & \\
\hline Stage 1 & 97 & 48.5 & 62 & 45.9 & 52 & 46.8 & 15 & 41.7 & 28 & 31.1 \\
\hline (Std. residual) & .9 & & .3 & & .4 & & -.2 & & -1.9 & \\
\hline Stage 2 & 52 & 26.0 & 47 & 34.8 & 30 & 27.0 & 11 & 30.6 & 29 & 32.2 \\
\hline (Std. residual) & -.9 & & 1.1 & & -.5 & & .1 & & .5 & \\
\hline
\end{tabular}




\begin{tabular}{|c|c|c|c|c|c|c|c|c|c|c|c|}
\hline$\frac{0}{\rho}$ & & & Length & time be & en disc & ery anc & agnosis & & & & \\
\hline & \multicolumn{3}{|c|}{$\underline{0-14 \text { days }}$} & \multicolumn{2}{|c|}{$15-30$ days } & \multicolumn{2}{|c|}{$31-60$ days } & \multicolumn{2}{|c|}{$61-90$ davs } & \multicolumn{2}{|c|}{$\geq 91$ days } \\
\hline & Stage at diagnosis & $\underline{\mathrm{n}}$ & $\%$ & $\underline{n}$ & $\%$ & $\underline{\mathrm{n}}$ & $\%$ & $\underline{\mathrm{n}}$ & $\%$ & $\underline{\mathrm{n}}$ & $\%$ \\
\hline & Stage 3 & 15 & 7.5 & 5 & 3.7 & 10 & 9.0 & 4 & 11.1 & 17 & 18.9 \\
\hline & (Std. residual) & -.7 & & -2.0 & & .0 & & .4 & & 3.2 & \\
\hline $\bar{~}$ & Stage 4 & 11 & 5.5 & 4 & 3.0 & 1 & .9 & 3 & 8.3 & 9 & 10.0 \\
\hline & (Std. residual) & .4 & & -1.0 & & -1.9 & & .9 & 2.2 & & \\
\hline & Total & 200 & 100 & 135 & 100 & 111 & 100 & 36 & 100 & 90 & 100 \\
\hline
\end{tabular}

Note. $X^{2}(16, N=572)=35.834, \underline{p}=.003$ 
Table 16

Relationship Between Stage at Diagnosis and Length of Time Between Diagnosis and Treatment

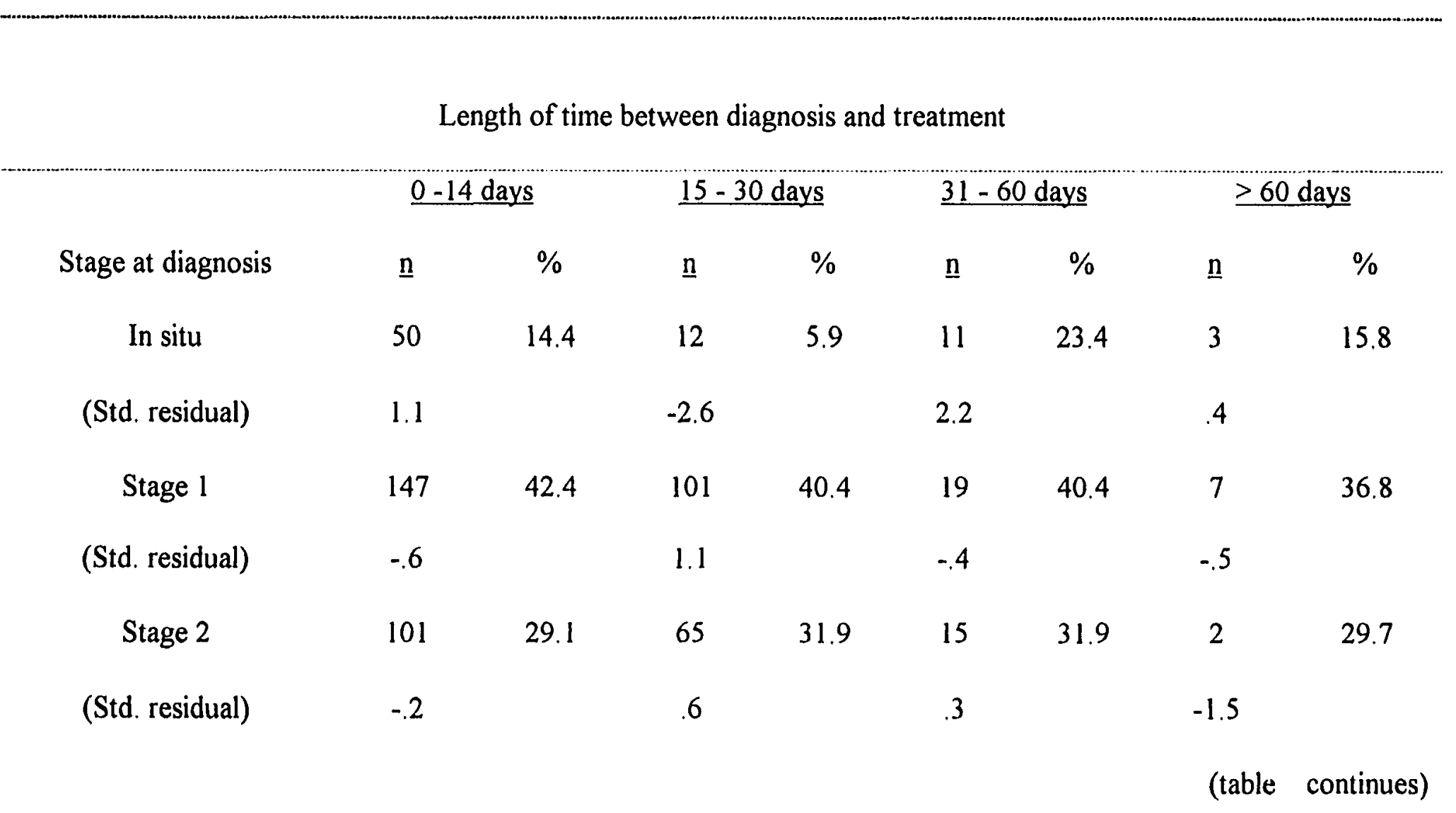


Length of time between diagnosis and treatment

\begin{tabular}{|c|c|c|c|c|c|c|c|c|}
\hline \multirow[b]{2}{*}{ Stage at diagnosis } & \multicolumn{2}{|c|}{$\underline{0-14 \text { days }}$} & \multicolumn{2}{|c|}{$15-30$ days } & \multicolumn{2}{|c|}{$31-60$ days } & \multicolumn{2}{|c|}{$\geq 60$ days } \\
\hline & $\underline{\mathrm{n}}$ & $\%$ & $\underline{\mathrm{n}}$ & $\%$ & $\underline{\mathrm{n}}$ & $\%$ & $\underline{\mathrm{n}}$ & $\%$ \\
\hline Stage 3 & 34 & 9.8 & 20 & 9.8 & 0 & 0 & 1 & 5.3 \\
\hline (Std. residual) & .6 & & .4 & & -2.0 & & -.5 & \\
\hline Stage 4 & 15 & 4.3 & 6 & 2.9 & 2 & 4.3 & 6 & 3.1 \\
\hline (Std. residual) & -.3 & & -1.2 & & -.1 & & 5.4 & \\
\hline Total & 347 & 100 & 204 & 100 & 47 & 100 & 19 & 100 \\
\hline
\end{tabular}

Note. $X^{2}(12, N=617)=53.330, p=.000$

Five cells $(25 \%)$ have an expected count of less than 5 . The minimum expected frequency is 89 . 
counts of less than five (Daniel, 1995; Norusis, 1997). The observed frequency of breast cancer cases diagnosed at stage 4 and having greater than 60 days between diagnosis and treatment $(3.1 \%$, std. residual $=5.4)$, at in situ and having 15 to 30 days between diagnosis and treatment $(5.9 \%$, std. residual $=-2.6)$, at in situ and having 31 to 60 days between diagnosis and treatment $(23.4 \%$, std. residual $=2.2)$, and at stage 3 and having 31 to 60 days between diagnosis and treatment, differed significantly from the expected frequency, and contributed significantly to the observed chi-square value.

Research Question 3: To what extent do the variables age, race, socioeconomic status, and the means of discovery of breast cancer predict the length of time between discovery and diagnosis of breast cancer, and the length of time between diagnosis and initiation of treatment of breast cancer, in women identifying their race as Caucasian or African American, and being diagnosed with breast cancer at Naval Medical Center San Diego from January 1, 1988 to December 31, 1997.

Standard multiple regression analysis to examine the relationships between age and the length of time between discovery and diagnosis, and the length of time between diagnosis and treatment; and to examine the extent to which the predictor variables of age, race, socioeconomic status, and means of discovery of breast cancer predict the length of time between discovery and diagnosis, and the length of time between diagnosis and treatment was obtained using SPSS.

Table 17 shows that there were no significant relationships between age at diagnosis of breast cancer and the length of time between discovery and diagnosis, and the length of time between diagnosis and treatment, $F(2,579)=2.129, p=.120$. 


\section{Table 17}

Relationship Between Age at Diagnosis and Variables of Realized Access $\mathrm{N}=582$

\begin{tabular}{lcccccc}
\hline Multiple R $=$ & 0.085 & & df & F & p \\
R Square $=$ & 0.007 & Regression & 2 & 2.129 & 0.120 \\
& & Residual & 579 & & \\
\hline Variable & & B & SE & Beta & p value \\
DISDIAG & $-5.139 \mathrm{E}-03$ & 0.004 & -0.055 & 0.189 \\
DIAGTRT & $4.799 \mathrm{E}-02$ & 0.030 & 0.066 & 0.111 \\
Constant & 57.628 & .744 & & \\
\hline
\end{tabular}

Key: DISDIAG $=$ Length of time between discovery and diagnosis DIAGTRT $=$ Length of time between diagnosis and treatment 
Table 18 shows multiple $\mathrm{R}, \mathrm{R}$ square, and the unstandardized regression coefficients (B) for the regression model, and the standardized regression coefficients $(\beta)$, the standard error (SE) for the unstandardized coefficients, and the $p$ value, for each of the predictor variables. The multiple $\mathrm{R}(.251)$ between the predictor variables (age, race, rank of sponsor, and means of discovery) and the outcome variable (length of time between discovery and diagnosis), was statistically significant, $F(9,573)=4.279, p=$ .000 . Six percent of the variance in the length of time between discovery and diagnosis was explained by the predictor variables. When taken separately, only incidental BSE $(\beta=$ $.255, p=.000)$ and $\operatorname{CBE}(\beta=.091, p=.033)$ were found to be significant predictors of the length of time in days between discovery and diagnosis of breast cancer.

Multicollinearity and singularity occur when variables are too highly correlated and can cause problems with interpretation of partial coefficients, sampling stability, and computational accuracy (Cohen \& Cohen, 1983). In order to eliminate high correlation and redundancy among variables, bivariate correlations greater than or equal to .70 should not be included in the analysis (Tabachnick \& Fidell, 1996).

Tolerance statistics can also be computed to assess multicollinearity among predictor (independent) variables. Tolerance is the proportion of variability of a predictor variable that is not explained by its relationships with the other predictor variables. Multicollinearity is a problem if tolerances are less than 0.1 (Norusis, 1997).

To assess the extent to which the predictor variables were independent of each other, tolerance statistics were calculated and an intercorrelation matrix was obtained (see Table 19). Tolerances ranged from .46 to .90 and were well above the 0.1 level, and none 
Table 18

General Regression of Age at Diagnosis, Race, Socioeconomic Status, and Means of Discovery on Length of Time Between Discovery and Diagnosis $\mathrm{N}=583$

\begin{tabular}{|c|c|c|c|c|c|}
\hline Multiple R & $=0$ & & & $\mathrm{~F}$ & $\underline{p}$ \\
\hline \multirow[t]{2}{*}{ R Square } & $=$ & \multicolumn{2}{|c|}{ Regression } & 4.279 & 0.000 \\
\hline & & \multicolumn{2}{|c|}{ Residual } & & \\
\hline \multicolumn{2}{|l|}{ Variable } & B & SE & Beta & $p$ value \\
\hline \multicolumn{2}{|c|}{ Age at diagnosis } & -0.119 & 0.472 & -0.011 & 0.801 \\
\hline \multicolumn{2}{|l|}{$\operatorname{Race}^{\mathrm{a}}$} & -21.442 & 21.504 & -0.043 & 0.319 \\
\hline \multicolumn{2}{|c|}{$\operatorname{Rank}(\mathrm{E} 1-\mathrm{E} 5)^{\mathrm{b}}$} & -40.699 & 28.183 & -0.069 & 0.149 \\
\hline \multicolumn{2}{|c|}{$\operatorname{Rank}(E 6-E 9)^{b}$} & -18.177 & 17.455 & -0.062 & 0.298 \\
\hline \multicolumn{2}{|c|}{$\operatorname{Rank}(C W O-04)^{b}$} & -7.612 & 21.341 & -0.019 & 0.721 \\
\hline \multicolumn{2}{|c|}{$\operatorname{Rank}(\mathrm{O} 5-\mathrm{O} 10)^{b}$} & -22.691 & 20.501 & -0.060 & 0.269 \\
\hline \multicolumn{2}{|c|}{ Intentional $\mathrm{BSE}^{\mathrm{c}}$} & 20.250 & 21.369 & 0.041 & 0.344 \\
\hline \multicolumn{2}{|c|}{${\text { Incidental } \mathrm{BSE}^{\mathrm{c}}}$} & 79.852 & 13.866 & 0.255 & $0.000^{*}$ \\
\hline \multicolumn{2}{|l|}{$\mathrm{CBE}^{\mathrm{c}}$} & 44.061 & 20.625 & 0.091 & $0.033 * *$ \\
\hline \multicolumn{2}{|l|}{ Constant } & 54.748 & \multicolumn{2}{|l|}{34.021} & \\
\hline
\end{tabular}

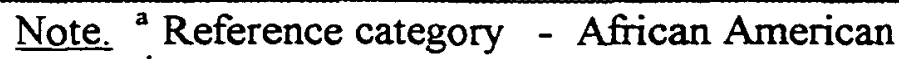

${ }^{b}$ Reference Category - Unknown

${ }^{c}$ Reference category - Mammogram

$*$ significant at $\mathrm{p} \leq .001 \quad * *$ significant at $\mathrm{p} \leq .05$ 
Table 19

Intercorrelation Matrix of the Variables of Potential Access (Length of Time Between Discovery and Diagnosis) $\mathrm{N}=583$

\begin{tabular}{|c|c|c|c|c|c|c|c|c|c|}
\hline Variable & Age & Race & Rank l & Rank 2 & Rank 3 & \multirow[t]{2}{*}{ Rank 4} & INTBSE & INCBSE & MAMM \\
\hline Age & 1.000 & & & & & & & & \\
\hline Race & $-0.231^{*}$ & 1.000 & & & & & & & \\
\hline Rank 1 & $-0.223^{*}$ & $0.189^{*}$ & 1.000 & & & & & & \\
\hline Rank 2 & -0.016 & 0.036 & $-0.239^{*}$ & 1.000 & & & & & \\
\hline Rank 3 & 0.024 & -0.104 & $-0.111 * *$ & $-0.385^{*}$ & 1.000 & & & & \\
\hline Rank 4 & $0.071 * *$ & $-0.119 * *$ & $-0.122^{*}$ & $-0.424^{*}$ & $-0.197^{*}$ & 1.000 & & & \\
\hline INTBSE & $-0.171^{*}$ & $0.080^{*}$ & 0.043 & -0.012 & -0.006 & -0.043 & 1.000 & & \\
\hline INCBSE & -0.155 & $0.099^{*}$ & $0.078 * *$ & 0.025 & 0.035 & $-0.076 * *$ & $-0.225^{*}$ & 1.000 & \\
\hline CBE & 0.059 & 0.065 & 0.025 & -0.046 & -0.017 & -0.024 & $-0.110^{* *}$ & $-0.221^{*}$ & 1.000 \\
\hline Note. & Key: & $\begin{array}{l}\text { Rank } 1 \\
\text { Rank } 3 \\
\text { INTBSE }\end{array}$ & $\begin{array}{l}\text { El - Es } \\
\text { CWO - } \\
\text { Intentio }\end{array}$ & $\begin{array}{l}\text { O4 } \\
\text { al BSE }\end{array}$ & $\begin{array}{c}\text { Rank } 2 \\
\text { Rank } 4 \\
\text { INCBSE }\end{array}$ & $\begin{array}{l}=\mathrm{E} 6 \\
=\mathrm{OS} \\
=\mathrm{In}\end{array}$ & $\begin{array}{l}\text { - E9 } \\
\text { - O10 } \\
\text { idental BSE }\end{array}$ & & \\
\hline
\end{tabular}


of the correlations in Table 19 were greater than .70 . Therefore multicollinearity was not considered a problem for these analyses.

Table 20 shows the multiple $\mathrm{R}, \mathrm{R}$ square, and the unstandardized regression coefficients (B) for the regression model, and the standardized regression coefficients $(\beta)$, the standard error (SE) for the unstandardized regression coefficients, and the $p$ value, for each of the predictor variables. The multiple $\mathrm{R}(.161)$ between the predictor variables (age, race, rank of sponsor, and means of discovery) and the outcome variable (length of time between diagnosis and treatment), approached the accepted .05 level of significance, $F(9,608)=1.793, p=.067$. Taken separately, age at diagnosis was found to be a significant predictor of the length of time between diagnosis and treatment $(\beta=.110, p=$ $.011)$. Rank of E1 - E5 $(\beta=.090, p=.058)$, intentional BSE $(\beta=.079, p=.067)$, and $\mathrm{CBE}(\beta=.078, \mathrm{p}=.065)$ approached the .05 significance level as predictors of the length of time between diagnosis and treatment. Table 21 is an intercorrelation matrix of the predictor variables. All correlations were less than .70 and tolerances ranged from .45 to .90 , therefore multicollinearity was not considered a problem for these analyses.

Research Question 4: To what extent do the variables age, race, socioeconomic status, and the means of discovery of breast cancer predict stage at diagnosis of breast cancer in women identifying their race as Caucasian or African American, and being diagnosed with breast cancer at Naval Medical Center San Diego from January 1, 1988 to December 31, 1997.

A direct logistic regression analysis was performed on stage at diagnosis (early vs. late) as an outcome, and age, race, SES, and means of discovery as predictor variables 
Table 20

General Regression of Age at Diagnosis, Race, Socioeconomic Status, and Means of Discovery on Length of Time Between Diagnosis and Treatment $\mathrm{N}=618$

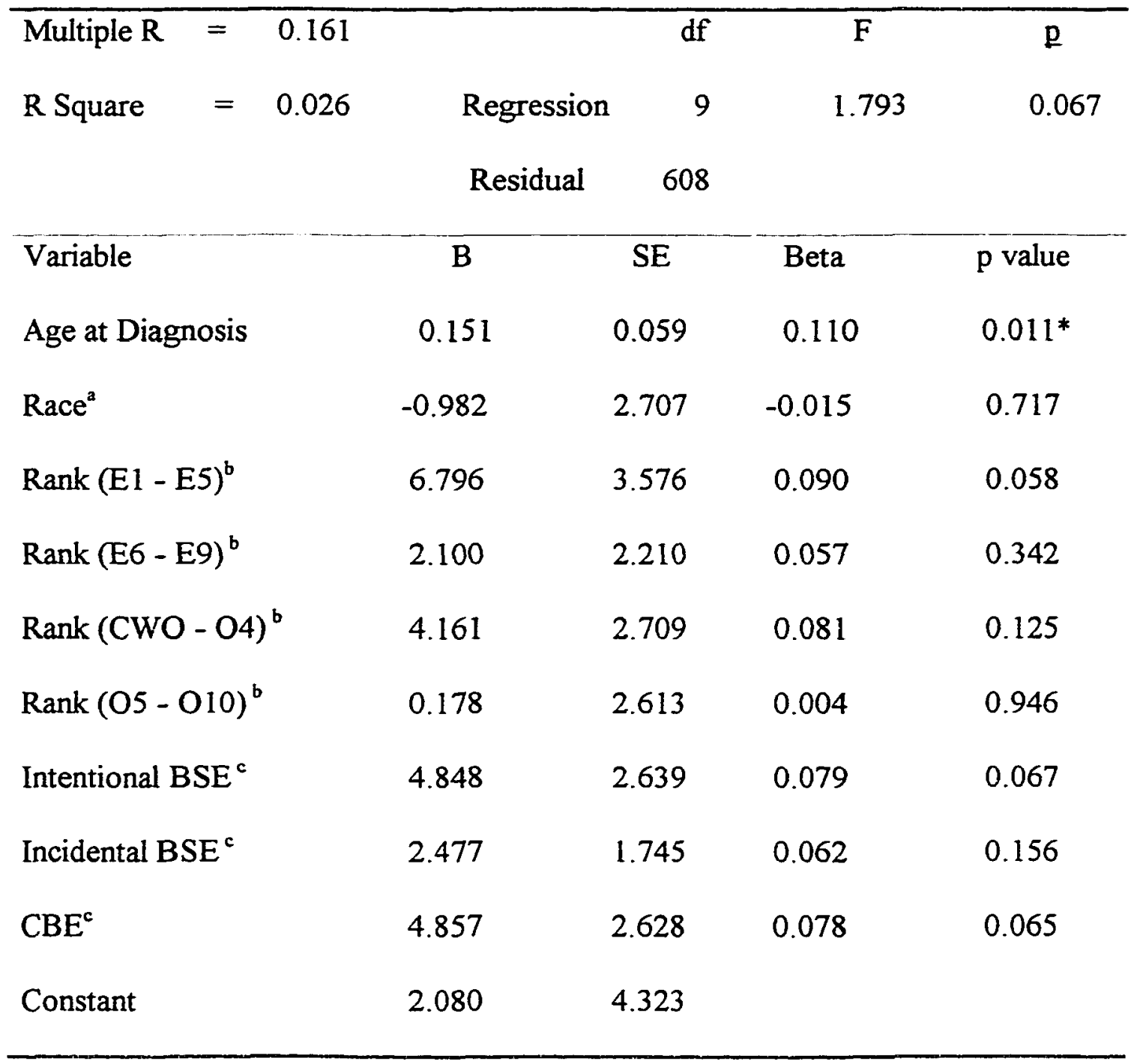

Note. a Reference category - African American

${ }^{b}$ Reference Category - Unknown

${ }^{c}$ Reference category - Mammogram

* significant at $\mathrm{p} \leq .05$ 
Table 21

Intercorrelation Matrix of the Variables of Potential Access (Length of Time Between Diagnosis and Treatment) $N=618$

\begin{tabular}{|c|c|c|c|c|c|c|c|c|c|}
\hline Variable & Age & Race & Rank 1 & Rank 2 & Rank 3 & Rank 4 & INTBSE & INCBSE & MAMM \\
\hline Age & 1.000 & & & & & & & & \\
\hline Race & $-0.231^{*}$ & 1.000 & & & & & & & \\
\hline Rank 1 & $-0.223^{*}$ & $0.189^{*}$ & 1.000 & & & & & & \\
\hline Rank 2 & -0.016 & 0.036 & $-0.239 *$ & 1.000 & & & & & \\
\hline Rank 3 & 0.024 & $-0.104^{* *}$ & $-0.111 * *$ & $-0.385^{*}$ & 1.000 & & & & \\
\hline Rank 4 & $0.071 * *$ & $-0.119 * *$ & $-0.122^{*}$ & $-0.424^{*}$ & $-0.197^{*}$ & 1.000 & & & \\
\hline INTBSE & $-0.171^{*}$ & $0.080^{* *}$ & 0.043 & -0.012 & -0.006 & -0.043 & 1.000 & & \\
\hline INCBSE & $-0.155^{*}$ & $0.099 * *$ & $0.078^{* *}$ & 0.025 & 0.035 & $-0.076 * *$ & $-0.225^{*}$ & 1.000 & \\
\hline CBE & 0.059 & 0.065 & 0.025 & -0.046 & -0.017 & 0.024 & $-0.110 * *$ & $-0.221 *$ & 1.000 \\
\hline Note. Key: & $\begin{array}{l}\text { Rank } 1 \\
\text { Rank } 3 \\
\text { INTBSE }\end{array}$ & $\begin{array}{l}=\mathrm{El} \\
=\mathrm{CV} \\
=\mathrm{Int}\end{array}$ & $\begin{array}{l}\text { - E5 } \\
\text { NO - O4 } \\
\text { entional BSE }\end{array}$ & & $\begin{array}{l}\text { nk 2 } \\
\text { nk } 4 \\
\text { CBSE }=\end{array}$ & $\begin{array}{ll} & \text { E6 - E9 } \\
\text { O5 - O10 } \\
=\quad \text { Incident }\end{array}$ & BSE & & \\
\hline
\end{tabular}

${ }^{*} \mathrm{p}<.001$ one tailed $\quad{ }^{* *} \mathrm{p}<.05$ one tailed 
using SPSS. After deletion of 18 cases with missing values, data from 617 women were available for analysis: 61 African American and 556 Caucasian.

A test of the full model with all of the predictors against a constant only model was statistically reliable, $\mathrm{X}^{2}(10, \mathrm{~N}=617)=126.358, \mathrm{p}=.000$, indicating that the predictors as a set, reliably distinguished between early (in situ, stage 1) and late (stages $2,3,4$ ) stage at diagnosis. In regard to prediction success, 70.57 percent of the early stage and 73.41 percent of the late stage breast cancer was correctly predicted, for an overall success rate of 71.80 percent.

Table 22 shows regression coefficients, Wald statistics, odds ratios and 95 percent confidence intervals for odds ratios, for each of the variables. Means of discovery was the only grouping which reliably predicted late stage at diagnosis, $z=88.0578, p=.0000$.

The odds for African American women $(\mathrm{OR}=1.06,95 \% \mathrm{CI} .57-2.00)$ being diagnosed with late stage breast cancer were slightly higher than the odds for Caucasian women $(\mathrm{OR}=1.00)$. Women with a sponsor's rank of $\mathrm{E} 1$ to $\mathrm{E} 5(\mathrm{OR}=2.11,95 \% \mathrm{CI} .87$ - 5.08) were three times more likely to be diagnosed at late stage breast cancer than women whose sponsor's rank was O5 - O10 (OR = .68, 95\% CI .36-1,29), and twice as likely to be diagnosed in late stage breast cancer than any of the other rank of sponsor groupings. Women who had their breast cancer discovered by incidental BSE ( OR = $3.79,95 \%$ CI 1.02 - 14.13) were almost six times more likely to be diagnosed at late stage breast cancer than women who had their breast cancer discovered by mammogram (OR = $.68,95 \% \mathrm{CI}=.36-1.29)$. 
Table 22

Logistic Regression Analysis of Late Stage at Diagnosis as a Function of Variables of Potential Access $\mathrm{N}=617$

\begin{tabular}{|c|c|c|c|c|c|}
\hline \multirow[b]{2}{*}{ Variables } & \multirow[b]{2}{*}{ B } & \multirow[b]{2}{*}{$\begin{array}{l}\text { Wald Test } \\
(z-\text { ratio })\end{array}$} & \multirow[b]{2}{*}{$\begin{array}{l}\text { Odds } \\
\text { Ratio }\end{array}$} & \multicolumn{2}{|c|}{$\begin{array}{c}95 \% \text { Confidence } \\
\text { Interval for Odds Ratio }\end{array}$} \\
\hline & & & & Lower & Upper \\
\hline Age & -0.0092 & 1.6803 & 0.99 & 0.98 & 1.00 \\
\hline \multicolumn{6}{|l|}{ Race } \\
\hline African American & 0.0626 & 0.0381 & 1.06 & 0.57 & 2.00 \\
\hline Caucasian $^{a}$ & & & 1.00 & & \\
\hline \multicolumn{6}{|l|}{ Rank of sponsor } \\
\hline $\mathrm{E} 1-\mathrm{E} 5$ & 0.7447 & 2.7457 & 2.11 & 0.87 & 5.08 \\
\hline E6 - E9 & 0.0906 & 0.1182 & 1.09 & 0.65 & 1.83 \\
\hline CWO - O4 & -0.0608 & 0.0353 & 0.94 & 0.50 & 1.77 \\
\hline $05-010$ & -0.3844 & 1.3869 & 0.68 & 0.36 & 1.29 \\
\hline Unknown Rank $^{a}$ & & & 1.00 & & \\
\hline \multicolumn{6}{|l|}{ Means of discovery } \\
\hline Intentional BSE & 1.0740 & 2.3313 & 2.93 & 0.74 & 11.62 \\
\hline Incidental BSE & $1.3319^{*}$ & 3.9338 & 3.79 & 1.02 & 14.13 \\
\hline $\mathrm{CBE}$ & -0.0608 & 0.0353 & 2.93 & 0.74 & 11.65 \\
\hline Mammogram & -0.3844 & 1.3869 & 0.68 & 0.36 & 1.29 \\
\hline Unknown means $^{\mathbf{a}}$ & & & 1.00 & & \\
\hline
\end{tabular}

Note. ${ }^{\mathrm{a}}$ Reference Category ${ }^{*} \mathrm{p} \leq .05$ 
Research Question 5: To what extent do the variables length of time between discovery and diagnosis of breast cancer, and length of time between diagnosis and initiation of treatment of breast cancer, predict stage at diagnosis of breast cancer in women identifying their race as Caucasian or African American, and being diagnosed with breast cancer at Naval Medical Center San Diego from January 1, 1988 to December 31, 1997.

A direct logistic regression analysis was performed on stage at diagnosis (early vs. late) as an outcome and the length of time between discovery and diagnosis, and the length of time between diagnosis and treatment as predictors using SPSS. After deletion of 67 cases with missing values, data from 568 women were available for analysis.

A test of the full model with all of the predictors against a constant only model was statistically reliable, $\mathrm{X}^{2}(2, \mathrm{~N}=568)=28.928, \mathrm{p}=.000$, indicating that the predictors as a set, reliably distinguished between early (in situ, stage 1) and late (stages $2,3,4$ ) stage at diagnosis. In regard to prediction success, 90.74 percent of the early stage and 20.49 percent of the late stage breast cancer was correctly predicted for an overall success rate of 60.56 percent.

Table 23 shows regression coefficients, Wald statistics, odds ratios and 95 percent confidence intervals for odds ratios for each of the variables. Only length of time between discovery and diagnosis reliably predicted late stage diagnosis, $z=17.3248, p=.0000$.

Presentation of Results from Supplemental Data Analysis

In order to examine: (a) the relationship between means of discovery and variables of potential access (race, age, SES); (b) the distribution of variables of potential access by age, (c) differences in age, tumor size, length of time between discovery and diagnosis, and length of time between diagnosis and treatment, by means of discovery (self 
Table 23

Logistic Regression Analysis of Late Stage at Diagnosis as a Function of Variables of Realized Access $\mathrm{N}=568$

$95 \%$ Confidence Interval for Odds Ratio

\begin{tabular}{lccccc} 
Variables & B & $\begin{array}{l}\text { Wald Test } \\
(z-\text { ratio })\end{array}$ & $\begin{array}{l}\text { Odds } \\
\text { Ratio }\end{array}$ & Lower & Upper \\
\hline DISDIAG & $0.0058^{*}$ & 17.3248 & 1.006 & 1.003 & 1.009 \\
DIAGTRT & -0.0029 & 0.3705 & 0.997 & 0.988 & 1.007 \\
\hline
\end{tabular}

Note. Key: DISDIAG $=$ Length of time between discovery and diagnosis DIAGTRT $=$ Length of time between diagnosis and treatment

$* \mathrm{p} \leq .001$ 
discovered, provider discovered); (d) the extent to which age, race, marital status, patient service status, and SES (rank of sponsor), predict self discovered (intentional and incidental BSE) and provider discovered (CBE, mammogram) breast cancer; and (e) the distribution of race, age, SES, means of discovery, and stage at diagnosis by year of diagnosis, additional data analyses were performed.

Relationship between means of discovery and variables of potential access. Comparative analyses of means of discovery and race, age, and SES are presented in Table 24. Independent samples chi-square tests were used to examine differences in frequency distributions, and standardized residuals were calculated to determine which cells contributed to any obtained significant $\mathrm{p}$ values.

As shown earlier in table in Table 14, and repeated in Table 24 for illustrative purposes, there was a significant difference in the distribution of means of discovery by race $(p=.000)$. Fifty-two percent of the Caucasian women included in the study had breast abnormalities discovered by mammogram, as compared to 21 percent of the African American women; and 46 percent of the breast abnormalities in the African American women were discovered by incidental BSE, as compared to 30 percent of the Caucasian women.

There was a significant difference in the means of discovery by age $(p=.000)$. Fifty-six percent of the women less than 40 years old had their cancers discovered by incidental BSE, as compared to 20 percent who had cancers discovered by intentional BSE, 17 percent by mammogram, and 6 percent by CBE. Women $40-49$ years old had almost equal percentages of their cancers discovered by incidental BSE (37.2\%) and 
Table 24

Relationship Between Variables of Potential Access (Race, Age, SES) and Means of Discovery

\begin{tabular}{ccccccc}
\hline Characteristic/ Variable & Intentional BSE & Incidental BSE & CBE & Mammogram & p value \\
\hline $\begin{array}{l}\text { Race, \% (no. of cases) } \\
\text { African American }\end{array}$ & $17.5(10)$ & $45.6(26)$ & $15.8(9)$ & $21.1(12)^{* * *}$ \\
Caucasian & $9.2(52)$ & $29.8(169)$ & $9.3(53)$ & $51.7(293)$ & $.000^{*}$ \\
Age, \% (no. of cases) & $20.3(13)^{* * *}$ & $56.3(36)^{* * *}$ & $6.3(4)$ & $17.2(11)^{* * *}$ \\
$<40$ & $17.7(20)^{* * *}$ & $37.2(42)$ & $8.8(10)$ & $36.3(41)$ \\
$40-49$ & $6.6(8)$ & $28.9(35)$ & $10.7(13)$ & $53.7(65)$ \\
$50-59$ & $7.0(14)$ & $22.0(44)^{* * *}$ & $9.0(18)$ & $62.0(124)^{* * *}$ \\
$60-69$ & $5.6(7)$ & $30.2(38)$ & $13.5(17)$ & $50.8(64)$ \\
70 and above & & &
\end{tabular}




\begin{tabular}{llllll}
\hline Characteristic/ Variable & Intentional BSE & Incidental BSE & CBE & Mammogram & p value \\
Rank of sponsor, \% (no. of cases) & & & & \\
E1 - E5 & $15.0(6)$ & $45.0(18)$ & $12.5(4)$ & $27.5(10)$ \\
E6 - E9 & $9.6(27)$ & $32.6(92)$ & $8.2(23)$ & $49.6(140)$ \\
CWO - O4 & $9.4(9)$ & $34.4(33)$ & $9.4(9)$ & $46.9(45)$ \\
O5 - O10 & $7.3(8)$ & $23.6(26)$ & $8.2(9)$ & $60.9(67)$ \\
Unknown & $12.5(12)$ & $27.1(26)$ & $16.7(16)$ & $43.8(42)$
\end{tabular}

Note. All $p$ values are 2 sided and were derived from chi-square tests.

$*$ significant at $\mathrm{p} \leq .001 \quad * *$ significant at $\mathrm{p} \leq .05$ 
mammogram (36.3); and women 50 years and older had greater than 50 percent of their cancers discovered by mammogram.

The four means of discovery groupings differed significantly on the distribution by rank of sponsor $(p=.043)$. Forty-five percent of the discoveries in the E1 - E5 sponsor rank group occurred by incidental BSE, as compared to 34 percent in the CWO - O4 group, 33 percent in the E6 - E9 group, and 24 percent in the $05-010$ group. Sixty-one percent of the discoveries in the 05 - O10 sponsor's rank group occurred by mammogram, as compared to 50 percent in the E6 - E9 group, 47 percent in the CWO O4 group, and 28 percent in the El - E5 group.

Distribution of variables of potential access by age. Comparative frequencies of the distribution of race, SES and means of discovery, by age categories, are presented in Table 25. Thirty-one percent of the African American women included in the study were less than 40 years old as compared to 10 percent of the Caucasian women. Within the rank of sponsor grouping, 39.1 percent of the women in the E1 -E5 category were less than 40 years old, as compared to 9.8 percent of the E6 through E9, 10.2 percent of the CWO - 04, and less than one percent of the O5 through O10. Eighty-two percent of the incidental BSE cancer discoveries occurred in women 40 years or older. Within the intentional BSE means of discovery, 79.9 percent of the women were 40 years and older, and 96.4 percent of the mammogram discovered cancers occurred in women 40 years and older.

Differences in mean scores of selected study variables by means of discovery. Means of discovery was recoded into self discovered means (intentional and incidental BSE) and provider discovered means (CBE and mammogram) and the t-test for 
Table 25

Distribution of Variables of Potential Access (Race, SES, Means of Discovery), By Age $(<40$ and $>=40)$

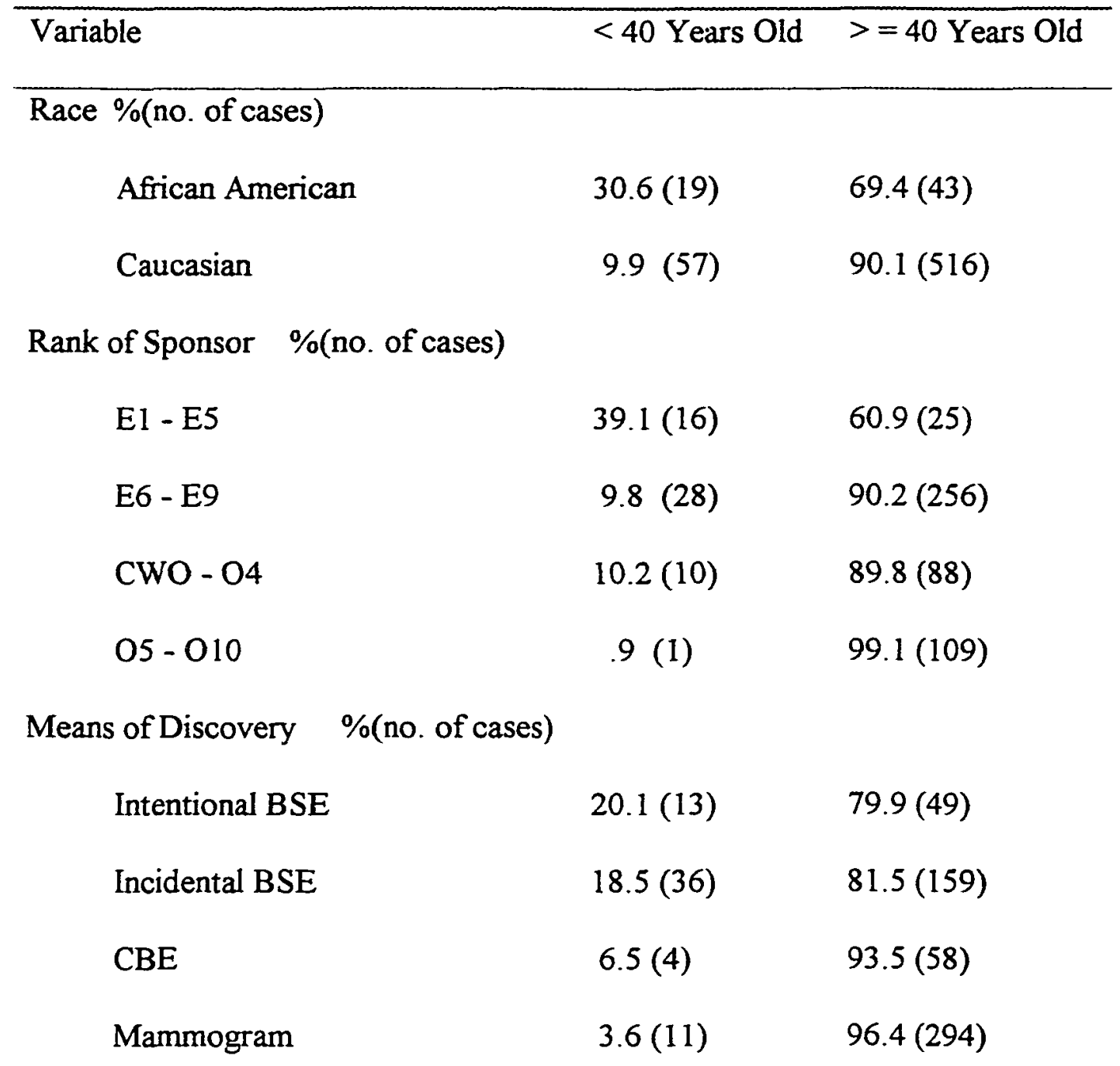


independent samples was used to test for significant differences between the mean scores of the two groups on age, tumor size, length of time between discovery and diagnosis, and length of time between diagnosis and treatment. A minimum sample size of a least 200 was achieved for all groups, therefore a power of greater than .995 was obtained using a medium effect size of .50 and a significance level of .05 (Cohen, 1988). Unequal variances, as determined by calculating the $F$ test, were treated by using the separate variance formula (Hinkle, Wiersma, \& Jurs, 1994).

Table 26 displays a summary of differences in mean scores of selected study variables, by self and provider discovered means. There were significant differences in means between the self discovered and provider discovered breast abnormalities on age at diagnosis $(t=-6.096 ; p=.000)$, size of tumor in centimeters $(t=6.820 ; p=.000)$, and length of time in days between discovery and diagnosis $(t=4.321 ; p=.000)$. There were no significant differences in the means between self discovered and provider discovered abnormalities on length of time in days between diagnosis and treatment $(t=1.047 ; p=$ $.295)$.

Extent to which age, race, patient service status, marital status, and SES predict seif or provider discovered means. Means of discovery was recoded into self discovered means (intentional and incidental BSE) and provider discovered means (CBE and mammogram), and age at diagnosis was categorized into five nominal groups. Marital status and patient service status were included as additional demographic descriptors of the women in this study. A direct logistic regression analysis was performed on means of discovery (self vs. provider) as an outcome, and age, race, marital status, patient service 
Table 26

T-Test Summary Table of Selected Study Variables by Means of Discovery

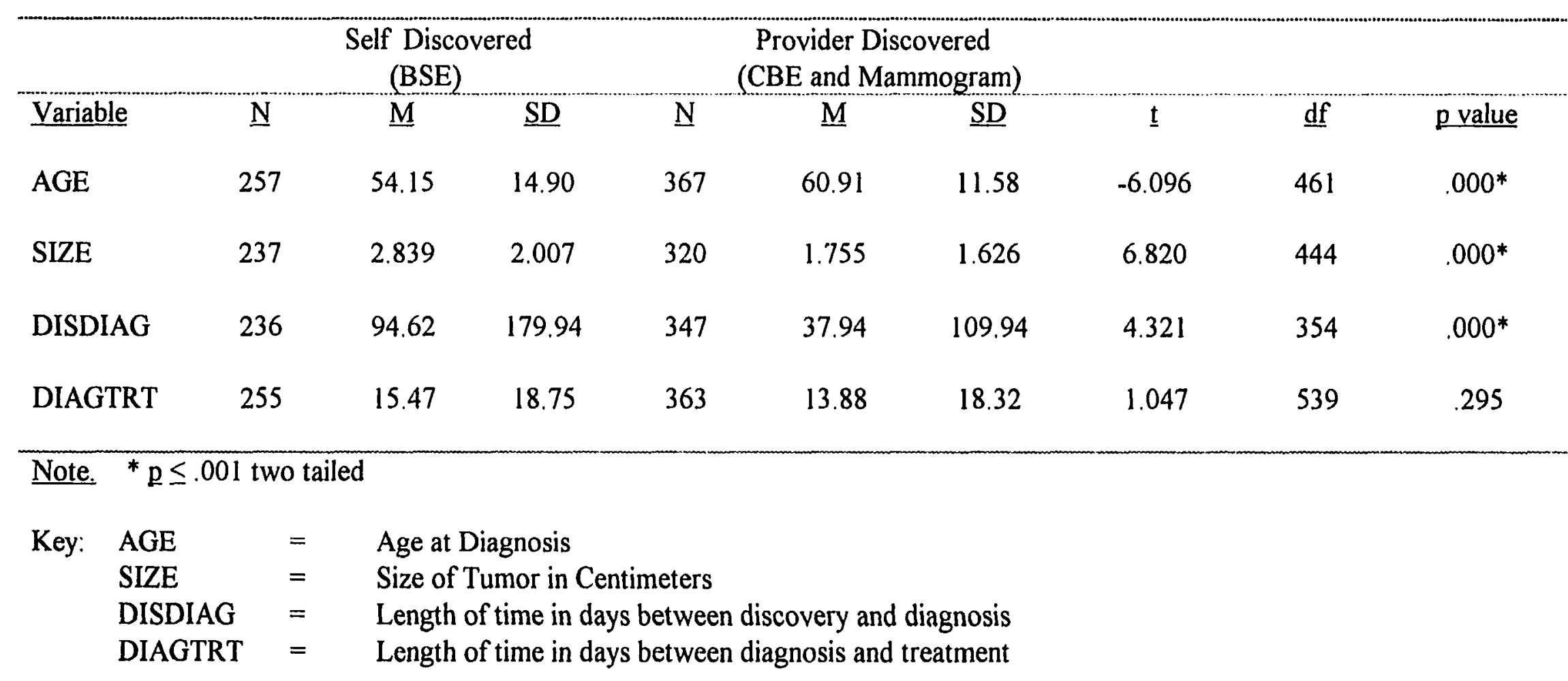


status, and SES as predictor variables, using SPSS. After deletion of 13 cases with missing values, data from 622 women were available for analysis.

A test of the full model with all of the predictors against a constant only model was statistically reliable, $\mathrm{X}^{2}(12, \mathrm{~N}=622)=64.992, \mathrm{p}=.000$, indicating that the predictors as a set, reliably distinguished between self (intentional and incidental BSE) and provider (CBE, mammogram) discovered means. In regard to prediction success, 41.80 percent of the self discovered abnormalities, and 83.06 percent of the provider discovered abnormalities were correctly predicted, for an overall success rate of 66.08 percent.

Table 27 shows regression coefficients, Wald statistics, odds ratios and 95 percent confidence intervals for odds ratios, for each of the variables. Age $(z=36.4114, p=$ $.0000)$ and race $(z=3.8065, p=.0511)$ reliably predicted provider discovered breast abnormalities.

Women 60 to 69 years old $(\mathrm{OR}=1.36,95 \% \mathrm{CI} .84-2.21)$ were seven times more likely to have provider discovered breast abnormalities, than were women less than 40 years old $(\mathrm{OR}=.20,95 \% \mathrm{CI} .091-.42)$; and three times more likely than women 40 to 49 years old $(\mathrm{OR}=.46,95 \% \mathrm{CI} .27-.80)$. The odds for women 60 to 69 years old having provider discovered breast abnormalities were also higher than for women 50 to 59 years old $(\mathrm{OR}=1.02,95 \% \mathrm{CI} .59-1.77)$, and for women 70 years and older $(\mathrm{OR}=$ 1.00).

Caucasian women $(\mathrm{OR}=1.00)$ were more likely to have provider discovered breast abnormalities than were African American women (OR = .54, 95\% CI .29 - 1.00). The odds for married women $(\mathrm{OR}=1.14,95 \% \mathrm{CI}=.73-1.77)$ having provider 
Table 27

Logistic Regression Analysis of Provider Discovered Breast Abnormality as a Function of Variables of Selected Demographic Variables $N=622$

\begin{tabular}{|c|c|c|c|c|c|}
\hline \multirow{2}{*}{ Variables } & \multirow{2}{*}{ B } & \multirow{2}{*}{$\begin{array}{l}\text { Wald Test } \\
\text { (z - ratio) }\end{array}$} & \multirow{2}{*}{$\begin{array}{l}\text { Odds } \\
\text { Ratio }\end{array}$} & \multicolumn{2}{|c|}{$\begin{array}{l}95 \% \text { Confidence } \\
\text { Interval for Odds } \\
\text { Ratio }\end{array}$} \\
\hline & & & & Lower & Upper \\
\hline \multicolumn{6}{|l|}{ Age } \\
\hline$<40$ & $-1.6283^{*}$ & 17.3136 & .20 & .091 & .42 \\
\hline $40-49$ & $-.7723 * *$ & 7.4602 & .46 & .27 & .80 \\
\hline $50-59$ & .0230 & .0068 & 1.02 & .59 & 1.77 \\
\hline $60-69$ & .3077 & 1.5375 & 1.36 & .84 & 2.21 \\
\hline$\geq 70^{\mathrm{a}}$ & & & 1.00 & & \\
\hline \multicolumn{6}{|l|}{ Race } \\
\hline African American & $-.6183^{* *}$ & 3.8065 & .54 & .29 & 1.00 \\
\hline Caucasian $^{2}$ & & & 1.00 & & \\
\hline \multicolumn{6}{|l|}{ Marital Status } \\
\hline Married & .1280 & .3226 & 1.14 & .73 & 1.77 \\
\hline Unmarried $^{\mathrm{a}}$ & & & 1.00 & & \\
\hline \multicolumn{6}{|l|}{ Patient Service Status } \\
\hline Active Duty & .0886 & .0319 & 1.09 & .41 & 2.89 \\
\hline Retired & -.1279 & .0388 & .88 & .25 & 3.14 \\
\hline Family Member ${ }^{2}$ & & & 1.00 & & \\
\hline & & & & (table & ntinues) \\
\hline
\end{tabular}




\begin{tabular}{lccccc}
\hline Variables & B & $\begin{array}{c}\text { Wald Test } \\
(\mathrm{z}-\text { ratio })\end{array}$ & $\begin{array}{c}\text { Odds } \\
\text { Ratio }\end{array}$ & $\begin{array}{c}\text { Lower } \\
\text { Interval for Odds } \\
\text { Ratio }\end{array}$ & Upper \\
\hline Rank of sponsor & -.2232 & .2716 & .80 & .35 & 1.85 \\
E1 - E5 & -.1175 & .2104 & .89 & .54 & 1.47 \\
E6 - E9 & -.1937 & .3860 & .82 & .45 & 1.52 \\
CWO - O4 & .2183 & .4881 & 1.24 & .67 & 2.30 \\
O5 - O10 & & & 1.00 & & \\
Unknown Rank & & & & & \\
\hline
\end{tabular}

Note. ${ }^{a}$ Reference

$* \mathrm{p} \leq .001 * * \mathrm{p} \leq .05$ 
discovered breast abnormalities were higher than the odds for unmarried women (OR $=$ $1.00)$; and active duty women ( $\mathrm{OR}=1.09,95 \% \mathrm{CI} .41-2.89)$ were more likely to have provider discovered breast abnormalities than were women who were family member beneficiaries $(\mathrm{OR}=1.00)$, or women who were retired from active duty $(\mathrm{OR}=.88,95 \%$ CI .25 - 3.14). The odds for women with a sponsor's rank of $\mathrm{O} 5$ to $\mathrm{O} 10(\mathrm{OR}=1.24$, $95 \%$ CI $.67-2.30$ ) having provider discovered breast abnormalities were higher than the odds for women with a sponsor's rank of E1 to E5 (OR $=.80,95 \% \mathrm{CI} .35-1.85)$, CWO to $\mathrm{O} 4(\mathrm{OR}=.82,95 \% \mathrm{CI} .45-1.52)$, and $\mathrm{E} 6$ to $\mathrm{E} 9(\mathrm{OR}=.89,95 \% \mathrm{CI} .54-1.47)$.

Distribution of race, age, SES, means of discovery, and stage at diagnosis by year of diagnosis. Independent samples chi-square tests were used to examine differences in distributions of race, age at diagnosis, rank of sponsor, stage at diagnosis (early versus late), and means of discovery, by year. There were no significant differences in distributions of race $X^{2}(9, \mathrm{~N}=635)=5.388, \mathrm{p}=.799$, age $X^{2}(36, \mathrm{~N}=635)=36.616, \mathrm{p}$ $=.440$, rank of sponsor $X^{2}(27, \mathrm{~N}=533)=32.096, \mathrm{p}=.229$, or stage at diagnosis $X^{2}(9$, $\mathrm{N}=617)=15.392, \mathrm{p}=.081$, by year. There was a significant difference in the distribution of means of discovery by year $X^{2}(27, \mathrm{~N}=624)=56.817, \mathrm{p}=.001$. Table 28 displays the trends in means of discovery from 1988 through 1997. In 1988 and 1989, the percentage of incidental BSE and mammogram discoveries were both 34.2 and 38.2 percent respectively. From 1992 through 1996, the percentage of mammogram discoveries consistently increased from 43.2 to 64.2 percent. Incidental BSE discoveries were at 39.2 percent in 1992, 20.6 percent in 1993, 35.8 percent in 1994, 25.4 percent in 
Table 28

\section{Distribution of Means of Discovery by Year}

\begin{tabular}{|c|c|c|c|c|c|c|c|c|}
\hline \multicolumn{9}{|c|}{ Means of Discovery } \\
\hline \multirow[b]{2}{*}{ Year of Diagnosis } & \multicolumn{2}{|c|}{ Intentional BSE } & \multicolumn{2}{|c|}{ Incidental BSE } & \multicolumn{2}{|c|}{ CBE } & \multicolumn{2}{|c|}{ Mammogram } \\
\hline & $\underline{\mathbf{n}}$ & $\%$ & $\underline{\mathbf{n}}$ & $\%$ & $\underline{\mathrm{n}}$ & $\%$ & $\underline{\mathbf{n}}$ & $\%$ \\
\hline 1988 & 6 & 15.8 & 13 & 34.2 & 6 & 15.8 & 13 & 34.2 \\
\hline 1989 & 7 & 12.7 & 21 & 38.2 & 6 & 10.9 & 21 & 38.2 \\
\hline 1990 & 7 & 11.5 & 17 & 27.9 & 6 & 9.8 & 31 & 50.8 \\
\hline 1991 & 5 & 7.7 & 15 & 23.1 & 10 & 15.4 & 35 & 53.8 \\
\hline 1992 & 6 & 8.1 & 29 & 39.2 & 7 & 9.5 & 32 & 43.2 \\
\hline 1993 & 16 & 23.5 & 14 & 20.6 & 4 & 5.9 & 34 & 50.0 \\
\hline 1994 & 4 & 7.5 & 19 & 35.8 & 1 & 1.9 & 29 & 54.7 \\
\hline 1995 & 3 & 4.5 & 17 & 25.4 & 9 & 13.4 & 38 & 56.7 \\
\hline 1996 & 1 & 1.5 & 15 & 22.4 & 8 & 11.9 & 43 & 64.2 \\
\hline
\end{tabular}




\begin{tabular}{|c|c|c|c|c|c|c|c|c|}
\hline \multicolumn{9}{|c|}{ Means of Discovery } \\
\hline \multirow[b]{2}{*}{ Year of Diagnosis } & \multicolumn{2}{|c|}{ Intentional BSE } & \multicolumn{2}{|c|}{ Incidental BSE } & \multicolumn{2}{|c|}{$\underline{\mathrm{CBE}}$} & \multicolumn{2}{|c|}{ Mammogram } \\
\hline & $\underline{\mathrm{n}}$ & $\%$ & $\underline{\mathrm{n}}$ & $\%$ & $\underline{\mathrm{n}}$ & $\%$ & $\underline{\mathrm{n}}$ & $\%$ \\
\hline 1997 & 7 & 9.2 & 35 & 46.1 & 5 & 6.6 & 29 & 38.2 \\
\hline Total & 62 & & 195 & & 62 & & 305 & \\
\hline
\end{tabular}

Note. $X^{2}(27, N=624)=56.817, p=.001$

Percentage displayed within the $\%$ category is the percent within the year of diagnosis. 
1995, and 22.4 percent in 1996. Mammogram discoveries were at 38.2 percent in 1997 , as compared to 46.1 percent for incidental BSE discoveries.

\section{Summary}

This chapter presented the results of the data analysis for this study in four sections. The first section presented a description of the sample, and was followed by presentation of comparative descriptive analysis of the study variables and selected demographic characteristics, in section two. The third section presented statistical analysis related to each of the research questions, and the results of supplemental statistical analysis were presented in section four. 


\section{CHAPTER V}

\section{SUMMARY, DISCUSSION, IMPLICATIONS, AND CONCLUSION}

This chapter includes: a summary of the research design and method, discussion of the research findings, implications for nursing practice, education, and research, and the conclusion of the research study.

\section{Summary of the Research Design and Method}

The purpose of this descriptive comparative study was to examine the relationship between access and stage at diagnosis of breast cancer in African American and Caucasian women diagnosed with breast cancer at NMCSD between January 1, 1988 and December 31, 1997. The conceptual framework for this study: (a) was based on the Aday/Andersen Framework for the Study of Access, (b) asserted that in addition to economic access, there are additional delineators of access to breast health care; and (c) theorized that there are influential relationships between potential access, realized access, and stage at diagnosis of breast cancer, within an equal economic access health care system. Indicators of potential access were defined as race, age, socioeconomic status, and means of discovery of breast cancer. Indications of realized access were defined as the length of time between discovery of breast abnormality and diagnosis, and the length of time between diagnosis and treatment. 
The following research questions were addressed:

1. What are the relationships between age, race, socioeconomic status, the means of discovery of breast cancer, and stage at diagnosis of breast cancer in women identifying their race as Caucasian or African American, and being diagnosed with breast cancer at Naval Medical Center San Diego from January 1, 1988 to December 31, 1997.

2. What are the relationships between the length of time between discovery and diagnosis of breast cancer, and the length of time between diagnosis and initiation of treatment of breast cancer, and stage at diagnosis of breast cancer in women identifying their race as Caucasian or African American, and being diagnosed with breast cancer at Naval Medical Center San Diego from January 1, 1988 to December 31, 1997.

3. To what extent do the variables age, race, socioeconomic status, and the means of discovery of breast cancer predict the length of time between discovery and diagnosis of breast cancer, and the length of time between diagnosis and initiation of treatment of breast cancer, in women identifying their race as Caucasian or African American, and being diagnosed with breast cancer at Naval Medical Center San Diego from January 1, 1988 to December 31, 1997.

4. To what extent do the variables age, race, socioeconomic status, and the means of discovery of breast cancer predict stage at diagnosis of breast cancer in women identifying their race as Caucasian or African American, and being diagnosed with breast cancer at Naval Medical Center San Diego from January 1, 1988 to December 31, 1997.

5. To what extent do the variables length of time between discovery and diagnosis of breast cancer, and length of time between diagnosis and initiation of treatment of breast 
cancer, predict stage at diagnosis of breast cancer in women identifying their race as Caucasian or African American, and being diagnosed with breast cancer at Naval Medical Center San Diego from January 1, 1988 to December 31, 1997.

Data were collected from the tumor registry records of 573 Caucasian and 62 African American women, and were analyzed using SPSS for Windows. Descriptive statistics were used to summarize and describe the demographic characteristics of the sample and the study variables; and inferential statistics were used to examine the relationships between the study variables. The results of the study confirm the existence of non-economic delineators of access to breast health care, support the influential relationships between potential access, realized access, and stage at diagnosis of breast cancer, and provide some new insights into the disparity in stage at diagnosis of breast cancer between African American and Caucasian women. Discussion of the results of the study are presented in the next section of this chapter.

\section{Discussion}

\section{Descriptive Characteristics of the Sample and Study Variables}

The racial distribution of African American (9.8\%) and Caucasian (90.2\%) women in this study is representative of the racial distribution of African Americans (12\%) and Caucasians (84\%) in the United States population. In addition, the racial distribution of women included in the DoD MHS wide studies of breast cancer conducted by Zaloznik (1995; African American women $10.41 \%$; Caucasian women 89.58\%), and Wojcik, Spinks, and Optenberg (1998; African American women 10.6\%; Caucasian women 
$89.4 \%$ ) is consistent with the distribution of African American and Caucasian women in this study.

The significant difference in the mean age at the time of diagnosis of breast cancer, between the African American and Caucasian women in this study, concurs with studies conducted both within and outside of the DoD MHS. In this study, the mean age for African American women was 47.94 years as compared to 59.13 for Caucasian women, and 29 percent of the African American women were diagnosed at an age less than 40 years as compared to 8.7 percent of the Caucasian women. In the DoD-wide study conducted by Zaloznik (1995), 24 percent of the African American women were diagnosed at an age less than 40 years old as compared to 9 percent of the Caucasian women; and in the DoD-wide study conducted by Wojcik, et al. (1998), the mean age for African American women was 50 years as compared to 57.1 years for Caucasian women, and 22.8 percent of the African American women were diagnosed at an age less than 40 years as compared to 9.2 percent of the Caucasian women. In a study conducted outside of the DoD by Newman and Alfonso (1997), the mean age for African American women was 56 years as compared to 63 years for Caucasian women, and approximately one third of the African American women were diagnosed at an age less than 50 years, as compared to one fifth of the Caucasian women.

In relation to patient service status, the results of this study correspond to the results of the study conducted by Wojcik, et al. (1998). In the Wojcik et al. study, 90 percent of the women were family members of active duty members or retirees, and 4.9 percent of the women were active duty. Within the group of active duty women, 10.3 
percent were African American, as compared to 4.3 percent Caucasian. In this study, 93.4 percent of the women were family members, 4.7 percent active duty, and 16.1 percent of the women within the active duty group were African American as compared to 3.5 Caucasian.

African American women in this study were diagnosed at significantly later stages than were Caucasian women. Forty-one percent of the African American women in this study were diagnosed with tumors confined to the breast (early stage breast cancer), as compared to 58.5 percent of the Caucasian women. This finding is consistent with the percentages reported by other studies (Coates et al., 1990; Eley et al., 1994; Hunter et al., 1993; Mandelblatt et al., 1995; Roach et al., 1997; Simon \& Severson, 1996; Woịcik et al., 1998). Specifically, Landis, Murray, Bolden and Wingo (1999) reported that from 1989 to 1994,50 percent of the African American women diagnosed with breast cancer presented with tumors confined to the breast, as compared to 62 percent of the Caucasian women. Wells and Horm (1992) reported that 45.7 percent of the African American women they studied were diagnosed in early stage breast cancer, as compared to 52.1 percent of the Caucasian women; and Newman and Alfonso (1997) found that 21.3 percent of the African American women in their study presented at early stage breast cancer as compared to 49 percent of the Caucasian women.

In this study, African American women tended to have larger tumors at the time of diagnosis. This significant difference in tumor size is in keeping with the findings of other studies. Roach et al. (1997), Wojcik et al. (1998), and Zaloznik (1995), all reported that African American women had significantly larger tumors at the time of diagnosis, when 
compared to Caucasian women. However, neither this study, nor any of the studies presented in chapter two reported significant differences in the number of positive lymph nodes between the two groups of women.

The primary means of discovery for the women in this study was mammogram (45.4\%). The significant difference in mammogram discovered breast cancers, for the African American (21.1\% mammogram) and Caucasian (51.7\% mammogram) women in this study, will be discussed in detail under research question one.

The mean exact length of time in days between discovery and diagnosis of breast cancer was 45.15 for African American women and 45.55 for Caucasian women. When the exact length of time was combined with the calculated in-exact length of time between discovery and diagnosis, the mean for African American women became 57.96, as compared to a mean of 61.29 for Caucasian women. In keeping with the findings of studies reported in chapter two and conducted outside of the DoD MHS, there were no significant differences between African American and Caucasian women on length of time between discovery and diagnosis of breast cancer. However, neither the study by Zaloznik (1995), nor the study by Wojcik et al. (1998), conducted within the DoD MHS, investigated the length of time between discovery of breast abnormality and diagnosis of breast cancer.

The mean length of time in days between diagnosis and treatment for African American women in this study was 13.26 as compared to 14.56 for Caucasian women, and there were no significant differences between the two groups. The mean length of time between diagnosis and treatment for the African American and Caucasian women in this 
study, was however, shorter than the mean time reported by Wojcik et al.(1998), in which African American women were found to have a significantly shorter length of time between diagnosis and treatment (21.15 days), than were Caucasian women (29.51 days).

Surgery was the means of initial treatment in 94.3 percent of the women in this study, and there were no significant differences between African American (98.4\%) and Caucasian (94.7\%) women. This finding is consistent with the DoD wide study finding of Wojcik et al. (1998) in which surgery was the means of initial treatment for 93.8 percent of the African American women and for 90.3 percent of the Caucasian women.

In keeping with the finding that African American women are more likely to die from breast cancer than Caucasian women (Chaulk, Kazandijan, \& Pipesh, 1995; Coates et al., 1990; Eley et al., 1994; Wagner \& Schatzkin, 1994; Wojcik et al., 1998), 16.1 percent of the African American women in this study were deceased due to breast cancer at the time of data collection, as compared to 7.2 percent of the Caucasian women.

Research Question 1: What are the relationships between age, race, socioeconomic status, the means of discovery of breast cancer, and stage at diagnosis of breast cancer in women identifying their race as Caucasian or African American, and being diagnosed with breast cancer at Naval Medical Center San Diego from January 1, 1988 to December 31, 1997.

The significant finding in this study, regarding the relationship between late age and early stage at diagnosis, is in keeping with the finding of Newman and Alfonso (1997). In both studies, women 50 years of age and older were diagnosed at in situ (stage 0 ) and stage 1 at a higher percentage than women less than 50 years old. On the other hand, the 
finding relating to the distribution of stage by age and race in this study, was different from the finding in Newman and Alfonso's study. Both studies reported detecting a significant difference in stage at diagnosis between African American and Caucasian women, and in the distribution of stage by age within the group of Caucasian women. However, while Newman and Alfonso reported a significant difference in the distribution of stage at diagnosis between African American women less than 50 years old, and African American women 50 years of age and older, this study found that there was no significant difference in the distribution of stage by age in the group African American women.

The finding of a significant difference in the stage at diagnosis of breast cancer between the African American and Caucasian women in this study, echo the results of other studies examining the relationship between race and stage at diagnosis of breast cancer. In studies conducted by Hunter et al. (1993) Mandelblatt et al. (1995), and Newman and Alfonso (1997), African American women were found to be diagnosed at significantly later stages than Caucasian women. Hunter et al. attributed this disparity to access to health care, lifestyle, and antecedent medical experiences in the African American women, and suggested that higher stage at diagnosis was related to poorer access to health care and lower socioeconomic status (SES). Mandelblatt et al. also attributed the disparity in stage at diagnosis to health care access and SES. Mandelblatt et al. found that the odds for being diagnosed with late stage breast cancer were higher for women living in areas with low SES rankings, and that mammography capacity was a significant independent predictor of stage at diagnosis. On the other hand, Newman and Alfonso reported that the disparity in stage at diagnosis of breast cancer in the African 
American and Caucasian women in their study, did not appear to be related to economic access to health care. The majority of the African American women in the Newman and Alfonso study had access to and used private medical care; and the distribution of stage at diagnosis in African American women receiving care through the public clinic was similar to the distribution of those receiving care through the private practice setting.

Zaloznik (1995) reported finding no significant difference in stage at diagnosis between African American and Caucasian women receiving care in the DoD MHS. In a study conducted using the tumor registry records of 6414 Caucasian and 746 African American women entered in the DoD Automated Tumor Registry from 1976 to 1992 , Zaloznik reported that African American women were significantly younger than Caucasian women, had significantly larger tumors than Caucasian women, but were not diagnosed at significantly later stages than Caucasian women. This finding is interesting in light of the results of the study by Wojcik et al. reported in 1998 . Wojcik et al. examined the breast cancer survival status of 698 African American and 5879 Caucasian women with records entered in the DoD central tumor registry from 1975 to 1994 and found that African American women were significantly younger than Caucasian women at the time of diagnosis, had significantly larger tumors than Caucasian women, and were diagnosed at significantly later stages than Caucasian women. Neither of the studies conducted inside the DoD MHS examined the effects of SES, or means of discovery on stage at diagnosis, but Wojcik et al. asserted that factors other than economic access may contribute to diagnosis of African American women at younger ages and later stages of breast cancer. The findings of this study, relating to the relationship between age, race, and stage at 
diagnosis support the assertion made by Wojcik et al., and indicate that in this sample, delineators of access, other than economic, appear to be related to the disparity in age, race, and stage at diagnosis.

Approximately 93 percent of the women included in this study were family members of active duty members or retirees. Therefore, the rank of the sponsor is not considered to be an effective measure of military rank or educational level for 93 percent of the women in this study. However, the rank of the sponsor is viewed as an appropriate measure of the SES of the household of the woman being diagnosed with breast cancer, and is supported as an effective means of comparing and contrasting the SES of the women included in this study.

The relationship between SES and stage at diagnosis was significant. Junior enlisted (lower pay level than senior enlisted, junior officer, senior officer) were diagnosed at stages 2 and 3 at a higher percentage than senior enlisted, junior officers, and senior officers. This finding of late stage at diagnosis of breast cancer at lower SES rankings is consistent with other studies reported in chapter two (Franzini et al., 1997; Wagner \& Schatzkin, 1994; Wells \& Horm, 1992). However, prior studies on the relationship between SES and stage at diagnosis of breast cancer, within an equal economic access system, were not available for comparison. In addition, this disparity in stage at diagnosis could be related to age at diagnosis, since junior enlisted and their spouses are frequently younger than senior enlisted, junior officers and senior officers. Furthermore, the significantly higher percentage of African American women with a sponsor's rank of 
junior enlisted, as compared to Caucasian women, could also be related to the disparity in stage at diagnosis by SES.

The percentage of women diagnosed in early stage breast cancer, by mammogram, is significantly higher than the percentage of women diagnosed in early stage breast cancer by any other means. Forty-five percent of the stage 2 cancers were diagnosed by incidental BSE, as compared to 16 percent diagnosed by mammogram, and 21 percent of the stage 0 breast cancers were diagnosed by mammogram, as compared to five percent diagnosed by intentional BSE, and four percent diagnosed by incidental BSE. These findings support the existing literature on breast cancer screening which reports that early stage diagnosis is more likely to be associated with mammogram than with any other means.

The finding relating to the distribution of means of discovery by race was significant. Fifty-two percent of the Caucasian women in this study had their breast cancers discovered by mammogram as compared to 21 percent of the African American women; and 30 percent of the Caucasian women discovered their breast cancers by incidental BSE as compared to 46 percent of the African American women. Nine percent of the Caucasian women discovered their breast cancers by intentional BSE as compared to 18 percent of the African American women; and 9 percent of the cancers in the group of Caucasian women were discovered by $\mathrm{CBE}$, as compared to 16 percent in the group of African American women. Only one study examining the relationship between stage at diagnosis and means of discovery in African American and Caucasian women could be located for comparison with the findings of this study. However, the available studies on 
the breast cancer screening behaviors and practices of African American and Caucasian women provide an additional avenue for discussion of this study's findings, in relation to the means of discovery. Vernon et al. (1992), O'Malley, Earp, and Harris (1997), and Roberson (1997) found, when examining breast cancer screening behaviors in African American and Caucasian women, that Caucasian women were more likely to participate in mammography than African American women. In these studies, the difference in screening practice was attributed to cost and lack of physician referral. On the other hand, Douglass, Bartolucci, Waterbor, and Sirles (1995), and Michels, Carter, Taplin, and Kugler (1995) reported finding no significant difference in mammography participation between African American and Caucasian women.

In the one study located examining the relationship between means of discovery and stage at diagnosis, Benedict, Williams, and Hoomani (1996) found that 55.6 percent of the African American women had cancers discovered by mammogram as compared to 31.7 percent of the Caucasian women; even though the Caucasian women had higher rates for annual CBE and mammogram, than the African American women. In addition, Benedict et al. reported that 33.3 percent of the cancers in African American women were discovered by accident, as compared to 7.35 in the Caucasian women; and that 11.1 percent of the cancers in African American women were discovered by BSE as compared to 34.1 in the Caucasian women. Benedict et al. did not include stage 0 in their analysis, and stage at diagnosis, in relation to means of discovery, was not stratified by race. In addition, inferential statistics were not conducted because of sample size. Therefore, even though the study conducted by these investigators offers some insight into the relationship 
between stage at diagnosis and means of discovery for women receiving care outside of an equal economic access system, the comparability of the results with this study's findings is difficult because of research design and methodology.

The results of these studies both support and contrast the findings of this study. The majority of the studies available on means of discovery relate to self reported breast cancer screening behaviors and activities of women who have not had breast cancer; and the one study located on means of discovery in women diagnosed with breast cancer did not provide optimal comparability. Nevertheless, the significant difference in stage at diagnosis in the African American and Caucasian women in this study appears to be related to the means of discovery.

Research Question 2. What are the relationships between the length of time between discovery and diagnosis of breast cancer, and the length of time between diagnosis and initiation of treatment of breast cancer, and stage at diagnosis of breast cancer in women identifying their race as Caucasian or African American, and being diagnosed with breast cancer at Naval Medical Center San Diego from January 1, 1988 to December 31, 1997.

The finding of a significant relationship between length of time in days between discovery and diagnosis, and stage at diagnosis supports that increased length of time between discovery and diagnosis is associated with late stage at diagnosis. Women with greater than 90 days between discovery and diagnosis seemed to have poorer outcomes in relation to stage at diagnosis. Sixty-one percent of the women with greater than 90 days between discovery and diagnosis were diagnosed in late stage breast cancer. Women with 
61 to 90 days between discovery and diagnosis were equally distributed (50\%) between early and late stage at diagnosis; and women with 0 to 60 days between discovery and diagnosis were diagnosed at late stage breast cancer at a range of 37 to 42 percent. Even though there are no prior studies which specifically examine the relationship between stage at diagnosis and length of time between discovery and diagnosis, available for comparison with the findings of this study, the existing literature on breast cancer maintains that late stage diagnosis of breast cancer is associated with prolonged time between discovery and diagnosis; occurs more frequently in African American women; and is a result of lack of economic access to care. However, the existence of economic access to health care within the group of women in this study renders economic access barriers irrelevant as the primary cause of diagnosis delay, and support the existence of other access barriers to care. Furthermore, the African American women in this study were diagnosed at significantly later stages than the Caucasian women, yet there was no significant difference between the two groups on length of time between discovery and diagnosis.

The relationship between length of time between diagnosis and treatment and stage at diagnosis was significant. This finding is interesting in light of the fact that diagnosis, and assignment of stage, occur primarily before the period of time between diagnosis and treatment commences. This finding will be discussed further under research question number three.

Research Question 3. To what extent do the variables age, race, socioeconomic status, and the means of discovery of breast cancer predict the length of time between discovery and diagnosis of breast cancer, and the length of time between diagnosis and 
initiation of treatment of breast cancer, in women identifying their race as Caucasian or African American, and being diagnosed with breast cancer at Naval Medical Center San Diego from January 1, 1988 to December 31, 1997.

The group of variables comprising potential access accounted for six percent of the variance in length of time between discovery and diagnosis. Incidental BSE emerged as the variable explaining the greatest amount of variance, followed by $\mathrm{CBE}$; and none of the other variables of potential access contributed significantly to the variance in this model. The amount of explained variance is low, and there are no prior studies available for comparison. Still, one interpretation of the low explained variance might be the existence of several other undefined variables of potential access, which influence both means of discovery of breast cancer, and the length of time between discovery and diagnosis. In addition, unidentified personal and health care delivery system factors related to length of time between discovery of breast abnormality and initial contact with the health care delivery system might also impact length of time between discovery and diagnosis.

The findings of this study did not support the variables of potential access (age, race, SES, means of discovery), as a group, as significant predictors of the length of time between diagnosis and treatment of breast cancer. The regression model did approach the level of significance, and age at diagnosis, when taken separately was found to be a significant predictor of the length of time between diagnosis and treatment. However, in the regression model examining the relationship between age and realized access, age at diagnosis was not found to be significantly related to the length of time between diagnosis and treatment. 
The results discussed thus far in this chapter support that stage at diagnosis is significantly related to the variables of potential access. While Aday and Anderson (1974) maintain that potential access can be conceptualized as characteristics of the individual and/or the health care delivery system, in this study, potential access has been supported as a characterization of the individual. Even though statistical analyses have also demonstrated the existence of significant relationships between the variables of realized access and stage at diagnosis, length of time between discovery and diagnosis appears to be a characteristic of the individual related to the means of discovery, while length of time between diagnosis and treatment appears to be a characteristic of the health care institution related to economic access to health care. If this conjecture is true, variables of potential access, which define characteristics of the individual, would not be expected to significantly predict length of time between diagnosis and treatment within an equal economic access system; and variables defining means of discovery would be expected to significantly predict the length of time between discovery and diagnosis. Given the findings of this study, it can be posited that the results relating to the extent to which variables of potential access predict the length of time between diagnosis and treatment, are consistent with the other results of this study.

Research Question 4. To what extent do the variables age, race, socioeconomic status, and the means of discovery of breast cancer predict stage at diagnosis of breast cancer in women identifying their race as Caucasian or African American, and being diagnosed with breast cancer at Naval Medical Center San Diego from January 1, 1988 to December 31, 1997. 
Means of discovery was the only potential access variable which reliably predicted late stage diagnosis of breast cancer. Within the means of discovery grouping, incidental BSE was the strongest predictor, and women with breast cancers discovered by incidental BSE were almost six times more likely to be diagnosed at late stage than were women with breast cancers discovered by mammogram. This finding supports study results discussed earlier relevant to the significant relationship between stage at diagnosis and means of discovery, in that mammogram appears to be related to early stage diagnosis, while incidental BSE seems to be related to late stage diagnosis. Both intentional BSE and $\mathrm{CBE}$ were four times more likely to result in late stage diagnosis, when compared to mammogram. Therefore, overall, the results of this study confirm that early stage diagnosis is more likely to be associated with mammogram than with any other means of discovery.

African American women, in this study, were slightly more likely to be diagnosed with late stage breast cancer, when compared to the Caucasian women. This odds ratio supports this study's finding of a significant difference between the two groups on stage at diagnosis of breast cancer. In addition, the increased likelihood of African American women being diagnosed at a later stage seems to be related to a higher percentage of African American women having breast cancers discovered by incidental BSE, the means of discovery most associated with late stage diagnosis; and a higher percentage of Caucasian women having cancers discovered by mammogram, the means of discovery most associated with early stage diagnosis. 
Women with a sponsor's rank of $\mathrm{El}$ to $\mathrm{E} 5$ were three times more likely to be diagnosed with late stage breast cancer than were women with a sponsor's rank of O5 to 010 , and were twice as likely to be diagnosed in late stage than any of the other rank of sponsor groupings. These findings support the discussion presented earlier in this chapter, and reiterate the assertion that the stage at diagnosis disparity in SES may be related to stage at diagnosis disparities within the age and race variables.

According to the results discussed above, late stage diagnosis is more likely to be associated with African American women from low SES (sponsor's rank of E1 to E5), with their breast cancers discovered by some means other than mammogram. Once again, this study's findings confirm the association between potential access and stage at diagnosis, and echo the findings of much of the breast cancer disparity literature, which reports that late stage breast cancer is more likely to be diagnosed in African American women from low SES and with low mammogram usage. But if economic access is the primary cause of late stage diagnosis in African American women, the profile for women diagnosed with late stage breast cancer within an equal economic access system should be different, and it appears to be the same. Even though the finding of significant relationships between age, race, SES and stage at diagnosis has been repeatedly reported in the literature (Coates, et al., 1990; Eley, et al., 1994; Hunter, et al., 1993; Mandelblatt, et al., 1995; Newman \& Alfonso, 1997; Wojcik, et al., 1998), studies specifically investigating and reporting the relationship between means of discovery and stage of diagnosis are not available in the literature. In this study, significant findings involving the relationship between means of discovery and stage at diagnosis appear to have an 
underlying association with significant findings relating to race, age, SES and length of time between discovery and diagnosis. Therefore, additional studies are needed to explore the relationships between age, race, SES, means of discovery, and stage at diagnosis, both inside and outside of the DoD. Discussion relating to additional statistical analyses conducted with these variables, in this study, is presented later in this chapter.

Research Question 5. To what extent do the variables length of time between discovery and diagnosis of breast cancer, and length of time between diagnosis and initiation of treatment of breast cancer, predict stage at diagnosis of breast cancer in women identifying their race as Caucasian or African American, and being diagnosed with breast cancer at Naval Medical Center San Diego from January 1, 1988 to December 31, 1997.

Within the group of realized access variables, only the length of time between discovery and diagnosis reliably predicted late stage at diagnosis. This finding supports the discussion presented earlier under research question number 3.

\section{Supplemental Data Analysis}

The findings of this study: (a) identify significant relationships between age, race, SES, means of discovery and stage at diagnosis; (b) identify significant differences in age, SES, and means of discovery by race; (c) identify significant relationships between length of time between discovery and diagnosis, and length of time between diagnosis and treatment, and stage at diagnosis; (d) identify means of discovery (incidental BSE and $\mathrm{CBE}$ ) as a significant predictor of length of time between discovery and diagnosis; (e) identify means of discovery as a reliable predictor of late stage diagnosis; and ( $f$ ) identify 
the length of time between discovery and diagnosis as a reliable predictor of late stage breast cancer. Furthermore, the disparity in stage at diagnosis, between the African American and Caucasian women in this study, and the significant relationship between length of time between discovery and diagnosis and stage at diagnosis, seem to both be related to means of discovery. In light of these findings, and the suggestion of underlying relationships to means of discovery, supplemental data analyses were conducted.

Discussion relevant to these supplemental findings are presented below.

Comparative analyses reflect a significant difference in the distribution of means of discovery by race, age, and SES. Previous discussions have identified the significant difference in distribution of means of discovery by race, and supplemental analyses reflect similar differences in the distribution of means of discovery by age and sponsor's rank.

The American Cancer Society (ACS) and the DoD MHS breast cancer screening guidelines recommend annual mammography for women age 40 and older (American Medical Women's Association [AMWA], 1997). Therefore, the percentage of women less than 40 years old, having their cancers discovered by mammogram, is expected to be significantly less than those 40 years and older. But the ACS and DoD MHS also recommend monthly BSE, and CBE at least every 3 years for women 20 to 39 years old. Thus, age can be supported as a possible influencer of low mammography percentages in the women less than 40 , but the high percentage of incidental discoveries in women less than 40, as compared to intentional BSE and CBE, can not be readily related to age.

Previous discussions have already identified the significant difference in mean age at diagnosis between African American and Caucasian women. Therefore, the disparity in 
stage at diagnosis between the two groups might be related to this significant difference in age, which might be related to the significant difference in mammogram discovered breast cancers in the two groups, which might be related to the DoD MHS age guidelines for annual screening mammography. However, the number of African American women 40 years and older is almost four times the number having breast cancers discovered by mammogram, so age may be one influencer of the number of mammogram discovered breast cancers diagnosed in the African American women in this study, but additional influencers exist and must be identified. This assertion is supported by the finding that while 516 of the Caucasian women in the study were 40 years and older, only 293 of the breast cancers discovered in this group of women occurred by mammogram. In addition, within the 40 to 49 year old age group 37.2 percent of the cancers were discovered by incidental BSE as compared to 36.3 by mammogram, and 8.8 percent by CBE. Mammography is the optimal secondary prevention strategy for breast cancer, but some cancers palpated on CBE and/or BSE, may not be detected on mammography (AMWA, 1997). As a result, a certain percentage of cancers may only be found by CBE or BSE. However, the large percentage of cancers discovered by incidental BSE raise questions about routine breast cancer screening practices in all age groups.

The disparity in stage at diagnosis of breast cancer by sponsor's rank, may also be related to age. Women with a sponsor's rank of E1 through E5 were diagnosed at significantly later stages than any of the other groupings, and 39.1 percent of the women in the E1 to E5 group were less than 40 years old, as compared to 10.2 percent for the CWO through $\mathrm{O} 4$ group, 9.8 percent for the E6 to E9 group, and less than one percent 
for the $\mathrm{O} 5$ to $\mathrm{O} 10$ group. But, there were 25 women with a sponsor's rank of E1 to E5 40 years of age and older, and only 10 had cancers discovered by mammogram. Of the women falling into the E1 to E5 category, 16 were less than 40 years old, however, 18 of the women in the E1 to E5 category had cancers discovered by incidental BSE, 6 by intentional BSE, and 4 by CBE. The disparity in stage at diagnosis within the sponsor's rank group may be related to race, but 63.8 percent of the African American women in the study had a sponsor's rank of E6 to E9, as compared to 27.7 percent for E1 to E5; and women in the E1 to E5 group were twice as likely to be diagnosed at late stage breast cancer, when compared to women in the E6 to E9 group.

In this study, breast cancers discovered by intentional BSE had the same odds for being diagnosed at late stage as those discovered by CBE. But the odds for cancers discovered by incidental BSE being diagnosed at late stage were almost six times the odds for mammogram discovered cancers. In addition, self discovered (BSE) breast cancers were diagnosed at a significantly earlier age, when compared to provider discovered (CBE and mammogram) cancers. While there was no significant difference in mean length of time between diagnosis and treatment between self discovered and provider discovered breast cancers, women who had self discovered cancers had significantly larger tumors, and a significantly higher mean length of time between discovery and diagnosis. Furthermore, supplemental logistic analysis supported that Caucasian women, 50 years of age and older, with a sponsor's rank of $\mathrm{O} 5$ through $\mathrm{O} 10$, were most likely to have provider discovered breast cancers. 
Improvements in the diagnosis and treatment of breast cancer have emerged over the past 20 years (Chu et al., 1996), and the findings of significant relationships between the variables of potential access and stage at diagnosis may be influenced by significant differences in the distribution of these variables across the years. However supplemental analysis revealed that there were no significant differences in the distribution of race, age, SES, or stage, by year; and even though chi-square analysis demonstrated a significant difference in the distribution of means of discovery by year, the trends in mammography and incidental BSE discoveries appear to be inconsistent.

The additional analyses conducted in this study have confirmed means of discovery as a major influencer of the significant differences in stage at diagnosis by race, age, and SES, and have demonstrated that the women with self discovered breast cancers had poorer stage at diagnosis outcomes. Nevertheless, additional study is needed to explore the relationship between means of discovery and stage at diagnosis in other races, and in other health care institutions inside and outside of the DoD.

The existence of significant differences in means of discovery by race, age, and SES, within an equal economic access health care system, provide support for the theory that a woman's participation in breast health care is influenced by the perception of the availability, necessity, and appropriateness of care. Knowledge of the "need" for breast health care and the existence of the "economic means" to obtain that care may have little or no influence on a woman's expediency in seeking care, if the care is not perceived as necessary, appropriate, or available. A perception of the availability of breast health care is influenced by economic factors as well as location of care, fit with time schedules, fit 
with family and personal responsibilities, and fit with cultural beliefs, values, and expectations. A perception of the necessity of breast health care is influenced by incorporation of health promotion and disease prevention into definitions of health, symptomatology and cultural definitions of severity, and personal and family priorities. A perception of appropriateness of care is influenced by the fit of breast health care with cultural values, beliefs, and practices, interactions and relationships with providers of care, and previous experience associated with breast cancer and breast health care. These perceptions are generated through experiences resulting from the interaction of cultural, structural, and social factors, and assessment of these perceptions can provide insight into differences in means of discovery by age, race and SES. Therefore, additional studies are also needed to evaluate the knowledge of the "need" for breast health care, and the perception of access appropriateness, necessity, and availability.

In addition, studies are needed: to evaluate adherence to breast cancer screening guidelines by women receiving care within the DoD MHS; to identify other elements of potential access which may influence participation in breast cancer screening programs and care seeking behaviors; to explore differences in the length of time between discovery of breast abnormality and contact with the health care delivery system, and length of time between discovery of abnormality and diagnosis of breast cancer; and to explore factors associated with the length of time between first medical consultation and diagnosis of breast cancer. 


\section{Implications for Nursing}

The implications of the findings of this study for nursing practice, education, and research, along with recommendations for further research, are presented in this section.

\section{Nursing Practice}

The results of this study confirm the existence of non-economic access barriers to participation in breast cancer screening programs for some women. Within the DoD MHS, "easy" access did not exist for all women receiving breast health care, and while the African American women in this study seemed to have poorer stage at diagnosis outcomes, the implications of this study impact women of all ethnicities and age groups, and provide opportunities for improvement of nursing practice in all clinical settings.

Early detection is the best defense against late stage breast cancer diagnosis. Therefore, every effort should be employed to ensure that women inside and outside of the DoD MHS are aware of, and participate in health promotion and disease prevention activities related to breast health care. Existence of economic access to care, did not translate into utilization of care in this study. Variables of potential access, such as age, race, SES, and means of discovery, were significantly related to stage at diagnosis, and explained a small amount of the variance in length of time between discovery and diagnosis. Therefore, breast health promotion and disease prevention programs should focus on being economically available, perceptually necessary, and culturally appropriate.

In this study, there was no significant difference in the length of time between diagnosis and treatment, by race, or by means of discovery. These findings support the existence of equal economic access to "superior medical and diagnostic technology" for all 
eligible DoD MHS beneficiaries. But diagnosis and treatment cannot be initiated if the health care system is not utilized. Therefore, breast health care programs must also emphasize utilization of health care resources, and the steps to be taken when breast abnormalities are discovered.

The results of this study confirmed findings of other studies investigating the disparity in stage at diagnosis between African American and Caucasian women. According to this study, late stage breast cancer was more likely to be diagnosed in African American women, from low SES, and with low mammogram usage. Even though the implications presented above apply to all age groups and ethnicities, the observation that the profile for women with delayed diagnosis within this equal economic access health care system reflects the profile for women outside of this system has specific implications for provision of breast health care and education to African American women. The percentage of incidental discoveries of breast cancer for African American women was significantly high, when compared to Caucasian women; and since African American women were diagnosed at younger ages and with larger tumors, emphasis on practicing monthly BSE and routine CBE and mammogram, in this population of women, is essential. If late stage diagnosis and mortality are to be decreased, BSE instruction must include "the how to" and "the what to do", and nurses must incorporate assessment of the woman's definition of health, and attitudes about seeking care into this breast health care instruction. In addition, African American women must be consistently encouraged to seek out breast health care, and to participate in CBE, BSE, and mammogram, even in the absence of symptoms. 
This study has provided new insights into the relationship between access to care and stage at diagnosis, and has tremendous implications for nursing practice. Even though additional study and exploration is needed to further identify, describe, and verify noneconomic access barriers to breast health care, incorporation of the implications discussed above, into current nursing practice, will enhance the success of breast health care programs targeting women of all ethnicities and ages.

\section{Nursing Education}

Nursing education programs must continue to make health promotion and disease prevention an integral part of their curriculums. Approaches to teaching health promotion and disease prevention should promote breast health care; and breast health care curriculums should emphasize the impact of non-economic access barriers on participation in breast cancer screening.

In addition, nursing education programs should expand their perceptions of culturally appropriate care, and begin to view each nurse-client encounter as an opportunity for increasing cultural awareness, and gaining insight into client's definitions of health, and care seeking behaviors. In this study, characteristics of the individual (such as age, race, and SES) had significant relationships to both means of discovery and stage at diagnosis. Therefore, non-economic access barriers to participation in breast health care might be more readily identified by assessing the client's cultural perception of health care necessity, appropriateness, and availability.

Finally, educators of nurses should emphasize the importance of breast cancer screening in students of nursing, at all levels. Educational programs should help students 
evaluate their own adherence to ACS recommendations for breast cancer screening, and identify personal barriers to accessing breast health care. Enhancing awareness of the relationship between access and stage at diagnosis, and increasing personal participation in breast health care, through nursing education, will contribute significantly to decreasing the percentage of incidental breast cancer discoveries, and subsequent late stage diagnoses.

\section{Nursing Research}

The findings of this study suggest a revised conceptual model (Figure 2). Within this equal economic access system, the identified variables of potential access influenced length of time between discovery and diagnosis, but did not influence length of time between diagnosis and treatment. Both the variables of potential access and length of time between discovery and diagnosis influenced stage at diagnosis, but length of time between diagnosis and treatment, although a measure of realized access, seemed to be influenced primarily by stage at diagnosis of breast cancer.

The revised conceptual model for the study of access to breast health care for women within the MHS should be tested with other races serviced by NMCSD, and with African American and Caucasian populations at other DoD MHS institutions. Although studies conducted using an automated data base, across a number of institutions simultaneously, provide generalizability, studies focusing on means of discovery and length of time between discovery and diagnosis will be best conducted by the review of actual tumor registry records. Since the findings from this study relating to means of discovery and length of time between discovery and diagnosis were most significant, it is 
Figure 2. REVISED Framework for the Study of Access to Breast Health Care for Women in the Military Health System

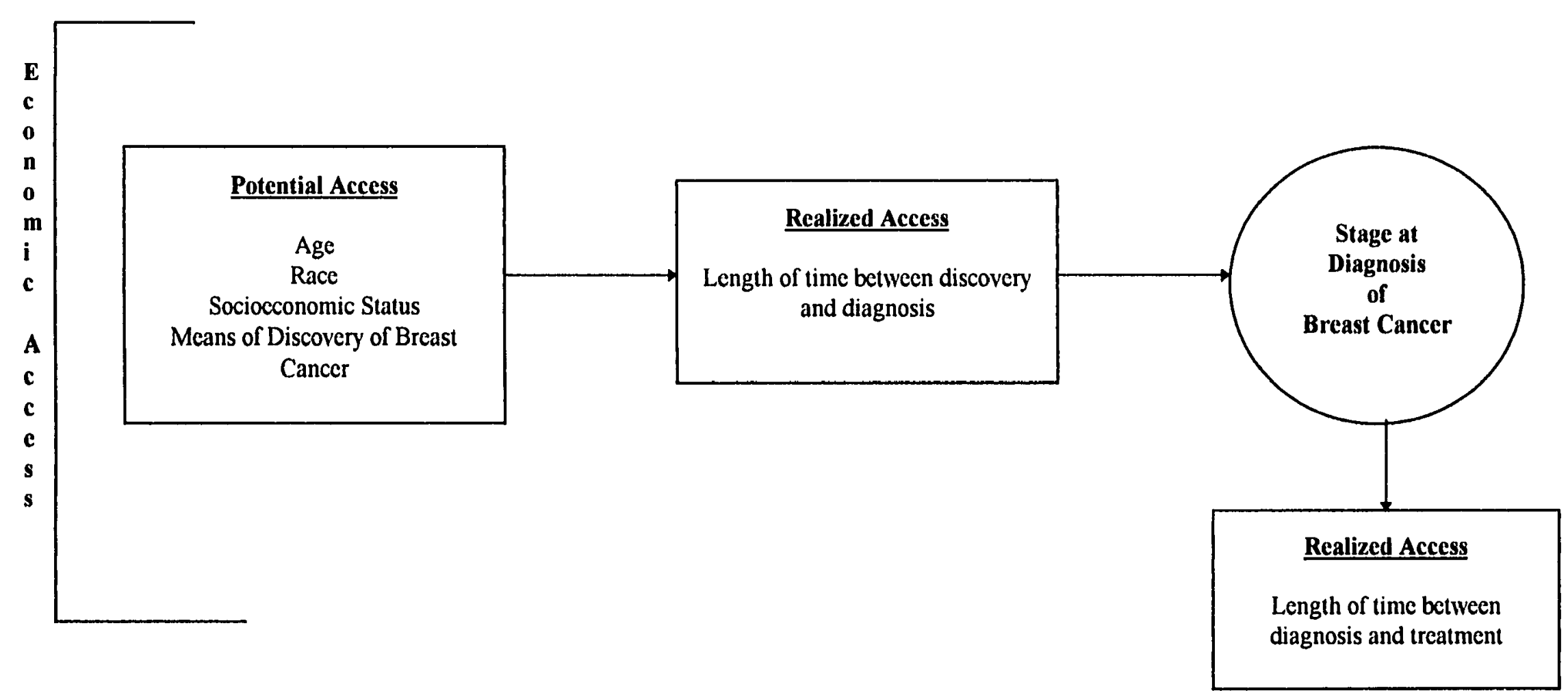

Adapted from Aday/Andersen Framework for the Study of Access (1974) 
recommended that these findings be confirmed by conducting studies within the MHS, using tumor registry records in conjunction with data bases.

Within the equal economic access health care system focused on in this study, barriers to access seemed to occur at the individual level (versus health care system level). The means of discovery influenced the timeliness of diagnosis, and questions surrounding the individual's ability and willingness to participate in health care arise from the poorer stage at diagnosis outcomes associated with self discovered breast cancer. In addition, the group of potential access variables identified in this study were only able to explain a small amount of the variance in length of time between discovery and diagnosis. Therefore, additional studies using the access framework and examining additional variables of potential and realized access are recommended. Within the potential access variable, additional predisposing factors such as family history of breast cancer and health promotion practices (alcohol use, tobacco use; body mass index) could be added, as well as other enabling factors such as geographic proximity to the health care institution, and transportation availability. Within the realized access variable, length of time between discovery and contact with the health care system should be evaluated in conjunction with length of time between discovery and diagnosis.

Stage at diagnosis influences both treatment and survival outcomes, and although this study did not examine treatment or survival outcomes in relation to stage at diagnosis, early stage diagnosis was associated primarily with mammogram, while late stage diagnosis was associated primarily with incidental BSE. Questions arise surrounding both of these means of discovery, but because mammogram discovered breast cancers were 
associated primarily with early stage diagnosis, it is recommended that additional studies be conducted within the DoD MHS describing the demographic characteristics and diagnostic outcomes of women receiving mammograms.

Even though this study was conducted with a sample of women at one institution, the demographic characteristics of this sample correspond to the demographic characteristics of studies conducted with populations of African American and Caucasian women both inside and outside of the DoD MHS. Therefore, it is recommended that the findings of this study be used to guide exploratory studies of a qualitative nature, inside and outside of the DoD, to identify other variables of potential access.

In addition, it is recommended that additional exploratory studies be conducted to further examine the significant relationship between age, race, SES, means of discovery, and stage at diagnosis. These studies should be conducted both inside and outside of the DoD MHS, and should explore these relationships in regard to potential access need factors such as health beliefs, health practices, and perceptions about the necessity, appropriateness, and availability of care.

Although this study added foundational knowledge on the relationship between access and stage at diagnosis of breast cancer, the additional studies discussed above are needed to substantiate the findings of this study, and develop hypotheses to be tested in future breast health care research. A summary of the recommendations for further study is presented below. 


\section{Summary of recommendations for further study.}

1. Conduct an expanded study, using the revised conceptual model, and comparing the findings of this study on African American and Caucasian women with data collected from the tumor registry records of Asian women, diagnosed with breast cancer at NMCSD.

2. Using the revised model, replicate this study at another large DoD MHS institution.

3. Conduct a study of the relationship between access and stage at diagnosis between African American and Caucasian women within the DoD MHS, using a revised framework which includes additional variables of potential and realized access.

4. Conduct a descriptive study of the demographic characteristics and diagnostic outcomes of women receiving mammograms within the DOD MHS.

5. Conduct qualitative exploratory studies inside and outside of the DoD MHS to identify other variables of potential access.

6. Conduct qualitative exploratory studies inside and outside of the DoD MHS to explore the relationships between potential access and stage at diagnosis, as they relate to health beliefs, health practices, and perceptions of access.

\section{Conclusion}

The findings of this study support the theorized relationships between the study variables. As in other studies examining the relationship between economic access and stage at diagnosis of breast cancer, the existence of economic access to care in this sample, did not always result in early diagnosis, and other factors such as age, race, SES, 
and means of discovery influenced diagnosis outcomes. Within this study, length of time between discovery and diagnosis was a function of means of discovery; and even though length of time between diagnosis and treatment was not predicted by potential access, this variable seemed to be a measure of equal access to treatment within this DoD MHS.

In this equal economic access system, there was a significant difference in stage at diagnosis between the African American and Caucasian women. Delayed diagnosis was the cause of this significant difference. African American women were diagnosed at later stages and with larger tumors, as compared to Caucasian women. However, this diagnosis delay did not appear to be related to economic access, in that there was no significant difference between the African American and Caucasian women in the length of time between diagnosis and treatment. The disparity in diagnosis delay between the African American and Caucasian women, and within the age and rank of sponsor categories, seemed to be related to the means of discovery. Self discovered breast cancers were associated primarily with late stage diagnosis, while provider discovered cancers were associated with early stage; and while intentional BSE and CBE had similar diagnostic outcomes, late diagnosis was most strongly associated with incidental BSE, while mammogram was most strongly associated with early diagnosis.

The results of this study clearly indicate that interventions aimed at increasing participation in breast health care programs among African American women, women of color, and all other categories of women, must focus on all aspects of access to heaith care. Economic access makes the difference from diagnosis to treatment, but treatment cannot be initiated until diagnosis occurs, and diagnosis cannot occur until access is 
realized. Therefore, breast health care interventions should be directed towards increasing awareness, increasing discovery, and decreasing the length of time between discovery and diagnosis, especially in women ages $20-40$. 


\section{References}

Adams, D. L. (Ed). (1995). Health issues for women of color: A cultural diversity perspective. Thousand Oaks: Sage.

Aday, L. A. (1985). Hospital sponsored medical groups: Their impact on access to primary care in rural communities. Journal of Community Health, 10 (3), 180-194.

Aday, L. A., \& Andersen, R. (1974). A framework for the study of access to medical care. Health Services Research, 9 (3), 208-220.

Aday, L. A., Andersen, R., \& Fleming, G. V. (1980). Health care in the US: Equitable for whom? Beverly Hills, CA: Sage.

Aday, L. A., Andersen, R., Loevy, S. S., \& Kremer, B. (1984). Hospital sponsored primary care: Impact on patient access. American Joumal of Public Health, 74 (8), 792-798

Aday, L. A., Fleming, G. V., \& Andersen, R. (1984). Access to medical care in the US: Who has it, who doesn't. Chicago, IL: Pluribus Press.

Aday, L. A., Lee, E. S., Spears, B., Chung, C. W., Youssef, A., \& Bloom, B. (1993). Health insurance and utilization of medical care for children with special health care needs. Medical Care, 31 (11), 1013-1026.

Adderley-Kelly, B., \& Green, P. M. (1997). Breast cancer education, self efficacy, and screening in older African American women. Journal of the National Black Nurses Association, 9 (1),45-57.

American Joint Committee on Cancer (AJCC). (1997). AJCC cancer staging manual (5th ed.). Philadelphia: Lippincott-Raven. 
American Medical Women's Association (AMWA). (1997). Breast cancer

Education for DoD primary care managers. Alexandria, VA: Author.

Andersen, R. M., Giachello, A. L., \& Aday, L. A. (1986). Access of Hispanics to health care and cuts in services: A state of the art overview. Public Health Reports, 101 (3), 238-252.

Ansell, D., Lacey, L., Whitman, S. Chen, E., \& Phillips, C. (1994). A Nurse delivered intervention to reduce barriers to breast and cervical cancer screening in Chicago inner city clinics.

Public Health Reports, 109 (1), 104-111.

Assistant Secretary of Defense for Health Affairs. (1996a, January). Policy guidelines for implementing managed care reforms in the military health services system. Washington, DC: Author.

Assistant Secretary of Defense for Health Affairs. (1996b, April). Breast cancer prevention, diagnosis, and education program. Washington, DC: Author.

Ayanian, J. Z., Kohler, B. A., Abe, T., \& Epstein, A. M. (1993). The relationship between health insurance coverage and clinical outcomes among women with breast cancer. The New England Journal of Medicine, 329 (5), 326-331.

Bakker, D. A., Lightfoot, N. E., Steggles, S., \& Jackson, C. (1998). The experience and satisfaction of women attending breast cancer screening. Oncology Nursing Forum, 25 (1), 115-121.

Bashshur, R. L., Homan, R. K., \& Smith, D. G. (1994). Beyond the uninsured: Problems in access to care. Medical Care, 32(5), 409-419. 
Bayne-Smith, M. (Ed.) (1996). Race, gender, and health. Thousand Oaks: Sage.

Bedell, M. B., Wood, M. E., Lezotte, D. C., Sedlacek, S. C., \& Orleans, M. M. (1995). Delay in diagnosis and treatment of breast cancer: Implications for education. Journal of Cancer Education, 10 (4), 223-228.

Benedict, S., Williams, R. D., \& Hoomani, J. (1996). Method of discovery of breast cancer. Cancer Practice, 4 (3), 147-155.

Berk, M. L., \& Schur, C. L. (1998). Measuring access to care: Improving information for policymakers. Health Affairs, 17 (1), 180-186.

Bibb, S. C. (1997). Breast self exam as a health promoting practice in African American women. Unpublished qualifying examination paper, University of San Diego.

Bowen, D., Hickman, K. M., \& Powers, D. (1997). Importance of psychological variables in understanding risk perceptions and breast cancer screening of African American women. Womens Health, 3 (3-4), 227-242.

Brink, P. J., \& Wood, M. J. (1994). Basic steps in planning nursing research: From question to proposal (4th ed.). Boston: Jones and Bartlett.

Brown, L. W., \& Williams, R. D. (1994, March). Culturally sensitive breast cancer screening programs for older black women. Nurse Practitioner, 21, 25-31.

Bunting, S. M., \& Campbell, J. C. (1994). Through a feminist lens: A model to guide nursing research. In P. L. Chinn (Ed.) Advances in methods of inquiry for nursing (pp. 75-87). Gaithersburg, Maryland: Aspen. 
Burnett, C. B., Steakley, C. S., \& Teff, M. C. (1995). Barriers to breast and cervical cancer screening in underserved women of the district of Columbia. Oncology Nursing Forum, 22 (10), 1551-1557.

Burns, N., \& Grove, S. K. (1993). The practice of nursing research, conduct, critique, and utilization (2nd ed.). Philadelphia: W. B. Saunders.

Caplan, L. S., Helzlsouer, K. J., Shapiro, S., Wesley, M. N., \& Edwards, B. K. (1996). Reasons for delay in breast cancer diagnosis. Preventive Medicine, 25 , 218-224. Champion, V. (1992). The Role of breast self exam in breast cancer screening. Cancer, 69 (7), 1985-1991.

Champion, V., \& Miller, A. M. (1996). Recent mammography in women aged 35 and older: Predisposing variables. Health Care for Women International, 17, 233-245.

Champion, V., \& Menon, U. (1997). Predicting mammography and breast self examination in African American women. Cancer Nursing, 20 (5), 315-322.

Champion, V. L., \& Scott, C. R. (1997). Reliability and validity of breast cancer screening belief scales in African American women. Nursing Research, 46 (6), 331-337.

Chaulk, C. P., Kazandjian, V. A., \& Pipesh, F. (1995). Racial differences in illness and mortality among Maryland women hospitalized with breast cancer under an all payer hospital system. Journal of Women's Health, 4 (1), 27-33.

Chinn, P. L. \& Kramer, M. K. (1995). Theory and nursing a systematic approach(4th ed.). St. Louis: Mossy. 
Chu, K. C., Tarone, R. E., Kessler, L. G., Ries, L. A., Hankey, B. F., Miller, B. A., \& Edwards, B. K. (1996). Recent trends in U. S. breast cancer incidence, survival, and mortality rates. Journal of the National Cancer Institute, 88 (21), 1571-1579.

Clark, J. Y., \& Thompson, I. M. (1994). Military rank as a measure of socioeconomic status and survival from prostate cancer. Southem Medical Journal, 87 (11), 1141-1144.

Coates, R. J., Bransfield, D. D., Wesley, M., Hankey, B., Eley, J. W., Greenberg, R. S., Flanders, D., Hunter, C. P., Edwards, B. K., Forman, M., Chen, V. W., Reynolds, P., Boyd, P., Austin, D., Muss, H., Blacklow, R. S., \& Black/White Cancer Survival Study Group. (1992). Differences between black and white women with breast cancer in time from symptom recognition to medical consultation. Joumal of the National Cancer Institute, 84 (12), 938-950.

Coates, R. J., Clark, W. S., Eley, J. W., Greenberg, R. S., Huguley, C. M., \& Brown, R. L. (1990). Race, nutritional status, and survival from breast cancer. Journal of the National Cancer Institute, 82 (21), 1684-1692.

Cohen, J. (1988). Statistical power analysis for the behavioral sciences (2nd ed.). Hillsdale, New Jersey: Lawrence Erlbaum.

Cohen , J., \& Cohen, P. (1983). Applied multiple regression/correlation analysis of the behavioral sciences (2nd ed.). Hillsdale, New Jersey: Lawrence Erlbaum.

Crane, J. (1995). The future of research utilization. Nursing Clinics of North America, 30 (3), 565-577. 
Crane, L. A., Kaplan, C. P., Bastani, R., \& Scrimshaw, S. C. (1996).

Determinants of adherence among health department patients referred for a mammogram. Women and Health, 24 (2), 43-64.

Crooks, C. E., \& Neutens, J. J. (1993). Prediction and verification of a woman's intention to participate in a mammography screening program. Journal of Health Education, 24 (4), 214-218.

Daniel, W. W. (1995). Biostatistics: A foundation for analysis in the Health Sciences. New York: John Wiley \& Sons.

DeBaca, V. (1994). Factors associated with women's decisions to obtain routine cervical cancer screening in a prepaid health care system (Doctoral dissertation, University of San Diego, 1994).

Defense Eligibility Enrollment Report System. (1998, March). Population by beneficiary category for Naval Medical Center San Diego (Fourth quarter fiscal year 1997). Defense Eligibility Enrollment Report System Database.

Department of Health and Human Services. (1990). Healthy people 2000: National health promotion and disease prevention objectives (DHHS Publication No. [PHS] 90-50212). Washington, DC: U. S. Government Printing Office.

Dibble, S. L., Vanoni, J. M., \& Miaskowski, C. (1997). Women's attitudes toward breast cancer screening procedures: Differences by ethnicity. Women's Health Issues, 7 (1), 47-54.

Donnelly, E. (1995). Culture and the meanings of cancer. Seminars in Oncology Nursing, 11, (1), 3-8. 
Douglass, M., Bartolucci, A., Waterbor, J., \& Sirles, A. (1995). Breast cancer early detection: Differences between African American and white women's health beliefs and detection practices. Oncology Nursing Forum, 22 (5), 835-837.

Duke, S. S., Gordon-Sosby, K., Reynolds, K. D.., \& Gram, I. T. (1994). A Study of breast cancer detection practices and beliefs in black women attending public health clinics. Health Education Research 9 (3), 331-342.

Eley, J. W., Hill, H. A., Chen, V. W., Austin, D. F., Wesley, M. N., Muss, H. B., Greenberg, R. S., Coates, R. J., Correa, P., Redmond, C. K., Hunter, C. P., Herman, A. A., Kurman,R., Blacklow, R., Shapiro, S., \& Edwards, B. K. (1994). Racial differences in survival from breast cancer. Journal of the American Medical Association, 272 (12), 947-954.

Erwin, D. O., Spatz, T. S., \& Turturro, C. L. (1992). Development of an African American role model intervention to increase breast self examination and mammography. Journal of Cancer Education, 7 (4), 311-319.

Facione, N. C. (1993). Delay versus help seeking for breast cancer symptoms: A Critical review of the literature on patient and provider delay. Social Science Medicine, $\underline{36}(12), 1521-1534$.

Facione, N. C., Dodd, M. J., Holzemer, W., \& Meleis, A. I. (1997). Helpseeking for self discovered breast symptoms: Implications for early detection. Cancer Practice, 5 (4), 220-227.

Facione, N. C., \& Facione. P. A. (1997). Equitable access to cancer services in the 21 st century. Nursing Outlook, 45(3), 118- 124. 
Fisher, L. D., \& Van Belle, G. (1993). Biostatistics: A methodology for the health sciences. New York: John Wiley \& Sons.

Franzini, L., Williams, A. F., Franklin, J., Singletary, S. E., \& Theriault, R. L. (1997). Effects of race and socioeconomic status on survival of 1332 black, Hispanic, and white women with breast cancer. Annals of Surgical Oncology, 4 (2), 111-118.

Frank-Stromborg, M., \& Olsen, S. J. (Eds.) (1993). Cancer prevention in minority populations: Cultural implications for health care professionals. St. Louis: Mosby.

Frazier, E. L., Jiles, R. B., \& Mayberry, R. (1996). Use of screening mammography and clinical breast examinations among black, Hispanic, and White women. Preventive Medicine, 25, 118-125.

Freeman, H., \& Wasfie, T. (1989). Cancer of the breast in poor black women. Cancer, 63, 2562-2569.

Freeman, H. Q. (1997). The meaning of race in cancer of the breast. The Cancer Journal from Scientific American, 3 (2), 76-77.

Friedman, E. (1994). Money isn't everything: Nonfinancial barriers to access. Journal of the American Medical Association, 271(19), 1535-1538.

Glenn, B. L., \& Moore, L. A. (1990). Relationship of self concept, health locus of control, and perceived cancer treatment options to the practice of breast self examination. Cancer Nursing, 13 (6), 361-365.

Griffin, F.N.U. (1994). Perceptions of African American women regarding health care. Journal of Cultural Diversity, 1 (2), 32-35. 
Grimm, L. G., \& Yarnold, P. R. (1995). Reading and understanding multivariate statistics. Washington, DC: American Psychological Association.

Guidry, J. J., Aday, L. A., Zhang, D., \& Winn, R. J. (1997). Transportation as a barrier to cancer treatment. Cancer Practitioner, 5 (6), 361-366.

Guidry, J. J., Greisinger, A., Aday, L. A., Winn, R. J., Vernon, S., \& Throckmorton, T. A. (1996). Barriers to cancer treatment: A review of published research. Oncology Nursing Forum, 23 (9), 1393-1398.

Hahn, R. A., \& Stroup, D. F. (1994). Race and ethnicity in public health surveillance: Criteria for scientific use of social categories. Public Health Reports, 109 (1), 7-15.

Han, Y., Baumann, L.C., \& Cimprich, B. (1996). Factors influencing registered nurses teaching breast self examination to female clients. Cancer Nursing, 19 (3), 197 203.

Harris, J. R., Lippman, M. E., Veronesi, U., \& Willett, W. (1992). Breast Cancer. New England Journal of Medicine, 327 (5), 319-328.

Heck, K. E., Wagener, D. K., Schatzkin, A., Devesa, S. S., \& Breen, N. (1997). Socioeconomic status and breast cancer mortality, 1989 through 1993: An Analysis of education data from death certificates. American Journal of Public Health, 87 (7), 12181222 .

Herman, A. A. (1996). Toward a conceptualization of race in epidemiologic research. Ethnicity and Disease, $6,7-20$. 
Hinkle, D. E., Wiersma, W., \& Jurs, S. G. (1994). Applied statistics for the behavioral sciences (3rd ed.). Boston: Houghton Mifflin.

Hoffman-Goetz, L. \& Mills, S. L. (1997). Cultural barriers to cancer screening among African American women: A Critical review of the qualitative literature. Womens Health, 3 (3-4), 183-201.

Huck, S. W., \& Cormier, W. H. (1996). Reading statistics and research (2nd ed.). New York: Harper Collins.

Hulley, S. B., \& Cummings, S. R. (1988). Designing clinical research: An epidemiologic approach. Baltimore: Williams \& Wilkins.

Hunt, J. M. (1996). Barriers to research utilization. Journal of Advanced Nursing, 23 (3), 423-425.

Hunter, C. P., Redmond, C. K., Chen, V. W., Austin, D. F., Greenberg, R. S., Correa, P., Muss, H. B., Forman, M. R., Wesley, M. N., Blacklow, R. S., Kurman, R. J., Digman, J. J., Edwards, B. K., \& Shapiro, S. (1993). Breast cancer: Factors associated with stage at diagnosis in black and white women. Journal of the National Cancer Institute. 85 (14), 1129-1137.

Jones, B. A., Kasi, S. V., Curnen, M. G., Owens, P. H., \& Dubrow, R. (1997). Severe obesity as an explanatory factor for the black/white difference in stage at diagnosis of breast cancer. American Journal of Epidemiology, 146 (5), 394-404.

Jennings, K. (1996). Getting black women to screen for cancer: Incorporating health beliefs into practice. Journal of the American Academy of Nurse Practitioners, 8 (2), 53-59. 
Kagawa-Singer, M. (1995). Socioeconomic and cultural influences on cancer care of women. Seminars in Oncology Nursing 11 (2), 109-119.

Kagawa-Singer, M. (1997). Addressing issues for early detection and screening in ethnic populations. Oncology Nursing Forum, 24 (10), 1705-1711.

Kato, P. M., \& Mann, T. (Eds.) (1996). Handbook of diversity issues in health psychology. New York: Plenum Press.

Katz, S. J., Hislop, T. G., Thomas, D. B., \& Larson, E. B. (1993). Delay from symptom to diagnosis and treatment of breast cancer in Washington state and British Columbia. Medical Care, 31 (3), 264-268.

Kirkwood, J. M., Lotze, M. T., \& Yasko, J. M. (1996). Current cancer therapeutics (2nd ed.). Philadelphia: Churchill Livingstone.

Krieger, N., Van Den Eeden, S. K., Zava, D., \& Okamoto, A. (1997). Race/ethnicity, social class, and prevalence of breast cancer prognostic biomarkers: A study of white, black, and Asian women in the San Francisco bay area. Ethnicity and Disease, 7 (2), 137-149.

Kurtz, M.E., Given, B., Given, C.W., \& Kurtz, J.C. (1993). Relationships of barriers and facilitators to breast self examination, mammography, and clinical breast examination in a worksite population. Cancer Nursing, 16 (4), 251-259.

Landis, S. H., Murray, T., Bolden, S., \& Wingo, P. A. (1999), Cancer statistics 1999. Cancer Journal for Clinicians, 49 (1),8-31.

Lauver, D. (1992a). A theory of care seeking behavior. Image, 24 (4), 281-287. 
Lauver, D. (1992b). Psychosocial variables, race, and intention to seek care for breast cancer symptoms. Nursing Research, 41 (4), 236-241.

Lauver, D., \& Angerame, M. (1993). Women's expectations about seeking care for breast cancer symptoms. Oncology Nursing Forum, 20 (3), 519-525.

Lauver, D., Coyle, M., \& Panchmatia, B. (1995). Women's reasons for and barriers to seeking care for breast symptoms. Women's Health Issues, 5 (1), 27-35.

Lauver, D., \& Ho, C. (1993). Explaining delay in care seeking for breast cancer symptoms. Journal of Applied Social Psychology, 23 (21), 1806-1825.

Lerman, C., Rimer, B., Trock, B., Balshem, A., \& Engstrom, P. F. (1990), Factors associated with repeat adherence to breast cancer screening. Preventive Medicine, $19,279-290$.

Lierman, L. M. (1988). Discovery of breast changes: Women's responses and nursing implications. Cancer Nursing 11 (6), 352-361.

Lierman, L.M., Young, H.M., Kasprzyk, D., \& Benoliel, J.Q. (1990). Predicting breast self examination using the theory of reasoned action. Nursing Research, 39 (2), 97 101.

Lierman, L.M., Young, H.M., Powell-Cope, G., Georgiadou, F., \& Benoliel, J.Q. (1994). Effects of education and support on breast self examination in older women. Nursing Research, 43 (3), 158-163.

Long, E. (1993). Breast cancer in African American women: Review of the literature. Cancer Nursing. 16 (1), 1-24. 
Mandelblatt, J., Andrews, H., Kerner, J. Zauber, A., \& Burnett, W. (1991).

Determinants of late stage of breast and cervical cancer: The impact of age, race, social class, and hospital type. Public Health Briefs, 81 (5), 646-649.

Mandelblatt, J., Andrews, H., Kao, R., Wallace, R., \& Kerner, J. (1995). Impact of access and social context on breast cancer stage at diagnosis. Journal of Health Care for the Poor and Underserved, 6 (3), 342-351.

Mandelblatt, J., Traxler, M., Lakin, P., Kanetsky, P., Kao, R., \& The Harlem Study Team. (1993). Targeting breast and cervical cancer screening to elderly poor black women: Who will participate?. Preventive Medicine, 22, 20-33.

McPherson, C. P., Swenson, K. K., Jolitz, G., \& Murray C. L. (1997). Survival of women ages 40 - 49 years with breast carcinoma according to method of detection. Cancer, 79 (10), 1923-1932.

Michels, T.C., Taplin, S. M., Carter, W. B., \& Kugler, J. P. (1995). Barriers to screening: The theory of reasoned action applied to mammography use in a military beneficiary population. Military Medicine, 160 (9),431-437.

Miller, A.M., \& Champion, V. L. (1996). Mammography in older women: One time and three year adherence to guidelines. Nursing Research, 45 (4), 239-245.

Miller, A.M., \& Champion, V. L. (1997). Attitudes about breast cancer and mammography: Racial, income, and educational differences. Women and Health, 26 (1), 41-63.

Miller, L. Y., \& Hailey, B.J. (1994). Cancer anxiety and breast cancer screening in African American women: A Preliminary study. Women's Health Issues, 4 (3), 170-174. 
Morgan, C., \& Levin, G. (1995). A Cancer prevention intervention for disadvantaged women: Design and implementation. Journal of Cancer Education, 10 (3), $168-175$

National Cancer Institute. (1996). NCI reports improvements in breast cancer death rates. National Cancer Institute [On-line]. Available: http://www.icic.nci.nih.gov.

Nemcek, M.A. (1990). Health beliefs and breast self examination among black women. Health Values, 14 (5), 41-52.

Newman, L. A., \& Alfonso, A. E. (1997). Age-related differences in breast cancer stage at diagnosis between black and white patients in an urban community hospital. Annals of Surgical Oncology, 4 (8), 655-662.

Northam, S. (1996). Access to health promotion, protection, and disease prevention among impoverished individuals. Public Health Nursing 13 (5), 353-364.

Norusis, M. J. (1997). SPSS: Guide to data analysis. New Jersey: Prentice Hall. O'Malley, M. S., Earph, J. L., Harris, R. P. (1997). Race and mammography in two north carolina counties. American Journal of Public Health 87 (5), 782-786.

Olsen, S. J., \& Frank-Stromborg, M. (1993). Cancer prevention and early detection in ethically diverse populations. Seminars in Oncology Nursing 9 (3), 198-209.

Olsen, S.J., \& Frank-Stromborg, M. (1994). Cancer prevention and screening activities reported by African American Nurses. Oncology Nursing Forum, 21 (3), 487494.

Olson, K.L., \& Morse, J.M. (1996). Explaining breast self examination practice. Health Care for Women International, 17, 575-591. 
Palos, G. (1994). Cultural heritage: Cancer screening and early detection. Seminars in Oncology Nursing, 10 (2), $104-113$.

Parker, S. L., Davis, K. J., Wingo, P. A., Ries, L. A., \& Heath, C.W. (1998). Cancer statistics by race and ethnicity. Cancer Journal for Clinicians, 48 (1), 31-48.

Pearlman, D. N., Rakowski, W., Ehrich, B., \& Clark, M. A. (1996). Breast cancer screening practices among black, hispanic, and white women: Reassessing differences. American Journal of Preventive Medicine, 12 (5), 327-337.

Pederson, L. M., \& Trigg, J. M. (1995). Breast cancer: A family survival guide. Westport, Connecticut: Bergin \& Garvey.

Persson, K., Svensson, P., \& Ek, A. (1997). Breast self examination: An analysis of self reported practice. Journal of Advanced Nursing, 25, 886-892.

Phillips, J. (1991). Cancer control by the year 2000: Implications for action. Journal of National Black Nurses Association, 5, 42-48.

Phillips, J.M., \& Wilbur, J. (1995). Adherence to breast cancer screening guidelines among African American women of differing employment status. Cancer Nursing, 18 (4), 258-269.

Polit, D. F., \& Hungler, B. P. (1995). Nursing Research: Principles and methods (5th ed.). Philadelphia: J. B. Lippincott.

Powell, I. J., Schwartz, K., \& Hussain, M. (1995). Removal of the financial barrier to health care: Does it impact on prostate cancer at presentation and survival? A comparative study between black and white men in a veterans affairs system. Urology, 46 (6), 825-830. 
Roach, M., \& Alexander, M. (1995). The prognostic significance of race and survival from breast cancer: A Model for assessing the reliability of reported survival differences. Journal of the National Medical Association, 87 (3), 214-219.

Roach, M., Cirrincione, C., Budman, D., Hayes, D., Berry, D., Younger, J., Hart, R., \& Henderson, I.C. (1997). Race and survival from breast cancer: Based on cancer and leukemia group b trial 8541. The Cancer Journal from Scientific American, 3 (2), $107-112$.

Roberson, N. L. (1997). Comparison of breast screening outcomes from a cancer control intervention for African American and white women in western New York. Journal of the Association for Academic Minority Physicians, 8 (2), 29-33.

Region Nine TRICARE (1998). Regional Health Services Plan. San Diego, Ca.: Author.

Sensiba, M. E., \& Stewart, D. S. (1995). Relationship of perceived barriers to breast self examination in women of varying ages and levels of education. Oncology Nursing Forum, 22 (8), 1265-1268.

Sheskin, D. J. (1997). Handbook of parametric and nonparametric statistical procedures. Boston: CRC Press.

Simon, M. S., \& Severson, R. K. (1996). Racial differences in survival of female breast cancer in the detroit metropolitan area. Cancer, 77 (2), 308-314.

Steiner, C. B. (1991). Access to cancer prevention, detection, and treatment. Cancer, 67, 1736-1740. 
Stevens, J. (1996). Applied multivariate statistics for the social sciences (3rd ed.). New Jersey: Lawrence Erlbaum.

Tabachnick, B. G., \& Fidell, L. S. (1996). Using multivariate statistics (3rd ed.). Northridge: Harper Collins.

Tessaro, I., Eng, E., \& Smith, J. (1994). Breast cancer screening in older African American women: Qualitative research findings. American Journal of Health Promotion, $\underline{8}(4), 286-293$.

Thomas, L.R., Fox, S.A., Leake, B.G., \& Roetzheim, R.G. (1996). The Effects of health beliefs on mammography utilization among a diverse sample of older women. Women and Health, 24 (3), 77-94.

Thompson, B., Montano, D. E., Mahloch, J., Mullen, M., \& Taylor, V. (1997). Attitudes and beliefs toward mammography among women using an urban public health hospital. Journal of Health Care for the Poor and Underserved, 8 (2), 186-201.

Urrutia-Rojas, X., \& Aday, L. A. (1991). A framework for community assessment: Designing and conducting a survey in a Hispanic immigrant and refugee community. Public Health Nursing, 8 (1), 20-26.

Valdini, A., \& Cargill, L. C. (1997). Access and barriers to mammography in new England community health centers. The Journal of Family Practice, 45 (3), 243-249.

Vernon, S. W., Tilley, B. C., Neale, A. V., \& Steinfeldt, L. (1985). Ethnicity, survival, and delay in seeking treatment for symptoms of breast cancer. Cancer, 55, 15631571. 
Vernon, S.W., Vogel, V.G., Halabi, S., Jackson, G.L., Lundy, R.O., \& Peters, G.N. (1992). Breast cancer screening behaviors and attitudes in three racial/ethnic groups. Cancer, 69 (1), 165-174.

Vogel, V. G. (1996). Assessing women's potential risk of developing breast cancer. Oncology, $10(10), 1451-1461$.

Wagner, D. K., \& Schatzkin, A. (1994). Temporal trends in socioeconomic gradient for breast cancer mortality among US women. American Journal of Public Health, 84(6), 1003- 1006.

Wells, B. L., \& Horm, J.W. (1992). Stage at diagnosis in breast cancer: Race and socioeconomic factors. American Journal of Public Health, 82 (10), 1383-1385.

Williams, G. A., Abbott, R. R., \& Taylor, D. K. (1997). Using focus group methodology to develop breast cancer screening programs that recruit African American women. Journal of community health, 22 (1), 45-56.

Williams, D.R., Lauizzo-Mourey, R., \& Warren, R.C. (1994). The concept of race and health status in America. Public Health Reports, 109 (1), 26-41.

Wojcik, B. E., Spinks, M. K., \& Optenberg, S. A. (1998). Breast carcinoma survival analysis for African American and White women in an equal-access health care system. Cancer, 82 (7), 1310-1318.

Zaloznik, A. J. (1995). Breast cancer stage at diagnosis: Caucasians versus AfroAmericans. Breast Cancer Research and Treatment, 34, 195-198.

Zaloznik, A. J. (1997). Breast cancer stage at diagnosis: Caucasians versus Hispanics. Breast Cancer Research and Treatment, 42, 121-124. 
APPENDICES 


\section{APPENDIX A \\ BREAST CANCER MEDICAL RECORD DATA COLLECTION SHEET}




\section{Breast Cancer Medical Record Data Collection Sheet}

Identification Number:

Race:

Date of Birth:

Patient Status: $\quad$ Active Duty Retired Family Member

Marital Status: $\quad$ Married Single Divorced Separated Widowed

Branch of Service Affiliation:

Rate/Rank:

Date of Diagnosis:

Age at Diagnosis:

$\begin{array}{lllllll}\text { Stage at Diagnosis: } & 0 & \text { I } & \text { II } & \text { III } & \text { IV }\end{array}$

Tumor Size in centimeters:

Number of Positive Lymph Nodes:

Means of Discovery:

Intentional Breast Self Exam

Incidental Breast Self Exam

Routine Clinical Breast Exam

Secondary Clinical Breast Exam

Routine Mammogram

Secondary Mammogram

Length of time in days between discovery of breast abnormality and diagnosis:

Date Discovered:

Length of time in days between diagnosis of breast cancer and initiation of treatment:

Date Treatment Initiated:

Means of Treatment: Surgery at the Time of Diagnosis

Surgery after Diagnosis

Radiation Therapy

Chemotherapy

Hormone Therapy

No Treatment

Survival Status at the Time of Data Collection:

Alive

Deceased Due to Other Cause
Deceased Due to Breast Cancer

Deceased Due to Unknown Cause 
APPENDIX B

HUMAN SUBJECTS LETTER NAVAL MEDICAL CENTER SAN DIEGO

Reproduced with permission of the copyright owner. Further reproduction prohibited without permission. 


\section{CLINICAI RESEARCE DEPARTM: :}

NAVAL MEDICAL CENTER

SAN DIEGO, CA 92134-5000

6500

AVA

11 Jun 98

From: Chairman, Scientific Review Committee (SRC)

Chairman, Committee for the Protection of Human Subjects

To: Commander

Via: Deputy Commander

Subj: EXPEDITED REVIEW OF CIP STUDY \#S-98-107, "FACTORS RELATED TO STAGE AT DIAGNOSIS OF AFRICAN AMERICAN AND CAUCASIAN WOMEN DIAGNOSED WITH BREAST CANCER IN THE MILITARY HEALTH SYSTEM"

Ref: (a) NSHSBETHINST 6000.41A

(b) NAVMEDCEN SDIEGOINST 6500.4D

1. The subject study was reviewed by members of the respective committees. In accordance with references (a) and (b), local expedited approval of the above protocol is requested effective the date of your endorsement below.

2. Local expedited approval was authorized for the above research project by two members of the SRC on 11 June 1998. The signature below is provided to reflect the approval by the conmittee members. This protocol was processed in accordance with part 2, section $A(i)$ of reference (a). This protocol is a retrospective chart review.

3. The Chairman, CPHS determined that the study is exempt from CPHS review. The signature below is provided to reflect that exemption.

D. L. REEVES

CDR, MSC, USN

Chairman, Scientific Review

Comnittee
K. DEAN GUBLER

CDR, MC, USN

Chairman, Committee for the Protection of Human Subjects

4. This expedited approval will allow the investigator to begin the study as soon as the minor changes requested by the reviewers have been submitted. An administrative entry will be included in the next set of committee minutes.

5. Approved/ Dored

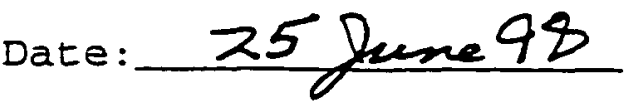

R. A. NELSON 


\section{DEPARTMENT OF CLINICAI RESEARCH \\ NAVAI MEDICAI CENTER \\ SAN DIEGO, CA 92134-5000}

6500

AVA

11 Jun 98

From: Head, Clinical Investigation Department (CID)

To: CAPT Sandra Bibb, NC, USN

SUbj: OFFICIAI APPROVAL OF CLINICAL INVESTIGATION PROGRAM (CIP) STUDY \#S-98-107 "FACTOR RELATED TO STAGE"AT DIAGNOSIS IN AFRICAN AMERICAN AND CAUCASIAN WOMEN DIAGNOSED WITH BREAST CANCER IN THE MILITARY HEALTH SYSTEM"

Ref: (a) NAVMEDCEN SANDIEGOINST 6500.4D

(b) NAVMEDCEN SANDIEGOINST 6500.5C

Encl: (1) Approved version of CIP Study

(2) Travel information

(3) Revisions to the protocol

1. Your research protocol (enclosure (I)) has been approved for initiation as of this date. Your protocol has met the requirements of references (a) and (b) and has been approved by the Scientific Review Committee (SRC). An annual review will be required in May 1999.

2. Requests for travel funds for presentations related to your protocol must be processed through Medical Editing and include your CIP \#. Travel funds are allocated by Naval School of Health Sciences (NSHS), Bethesda, $\mathrm{MD}$ and are available on a first come Eirst served basis. The information needed to apply for TAD funded by NSHS is in enclosure (2).

3. Articles/abstracts for publications must be submitted to the DCR Medical Editing staff. They will assist in the preparation, ensure proper acknowledgment of BUMED as sponsor, obtain command approval and submit them to journals and publications.

4. Changes to the protocol (i.e., increasing/decreasing subjects or budget, addition/deletion of investigators, and changes in protocol procedures) must be submitted to DCR for approval (enclosure (3)) prior to initiation.

5. Sincere best wishes for a successful research project. For assistance, please contact the Research Program Administration office at 532-8125/36.

\section{D: I. REEVES}


APPENDIX C

HUMAN SUBJECTS LETTER UNIVERSITY OF SAN DIEGO

Reproduced with permission of the copyright owner. Further reproduction prohibited without permission. 


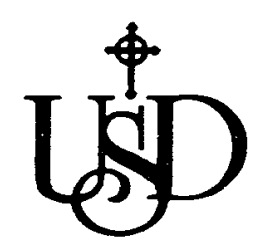

Universily of San Diego

Philip Y. Hahn School of Nursing

July 18,1998

Sandra Bibb DNSc (c)

Doctoral Student

Philip Y. Hahn School of Nursing

Dear Sandra:

I have received and reviewed your application to the USD Committee for the Protection of Human Subjects for your doctoral dissertation proposal. On July 17, 1998, I consulted with Dr. Gary Schneider, Chairperson of the Committee regarding the status of your application.

Both Dr. Schneider and I concur that your proposal as described is exempt from review by the Committee for the Protection of Human Subjects under the guidelines stipulated in the USD Faculty Handbook. Therefore, you may now proceed with the conduct of your dissertation.

I wish you success in your research endeavors. Please contact me if your require any additional information.

Sincerely,

Jane Georges, PhD, RN

Assistant Professor

School of Nursing Representative

Committee for the Protection of Human Subjects 